\title{
12. SHALLOW SUBMARINE TO EMERGENT BASALTIC SHIELD VOLCANISM OF GRAN CANARIA: EVIDENCE FROM DRILLING INTO THE VOLCANIC APRON ${ }^{1}$
}

\author{
Hans-Ulrich Schmincke ${ }^{2}$ and Beate Segschneider ${ }^{2}$
}

\begin{abstract}
Almost $500 \mathrm{~m}$ of basaltic hyaloclastite tuffs, hyaloclastite lapillistones, and lithic breccias were drilled in the northern, southeastern, and southwestern flank of Gran Canaria, Canary Islands during Leg 157 (Hole 953C, total penetration 1159 meters below seafloor [mbsf], basal $293 \mathrm{~m}$; Hole 954B, total penetration $446 \mathrm{mbsf}$, basal $38 \mathrm{~m}$; and Hole 956B, total penetration $704 \mathrm{mbsf}$, basal $140 \mathrm{~m}$ ). These deposits represent (1) mostly moderate to shallow water $(<<\sim 500 \mathrm{~m})$ eruptions, (2) transition to the emergence, and (3) the fully subaerial island shield stage. The volcaniclastic rocks are interlayered with minor thin layers of nannofossil ooze and clay.

Volcanic clasts comprise blocky to vesicular, generally altered former sideromelane shards, tachylite crystallized basalt, round glassy shards, lapilli, and single crystals, chiefly titanaugite. Dominantly filled foraminifers, thick-walled shallow-water skeletal debris, and nannofossil ooze make up $<5 \mathrm{vol} \%$ of the volcaniclastic rocks. Most of the basaltic volcaniclastic deposits are interpreted to have been deposited as debris flows resulting from (1) destabilization of hyaloclastites generated during voluminous moderate ( $<500 \mathrm{~m}$ ?) to shallow-water explosive volcanic activity and temporarily accumulated prior to episodic failure and transfer to the deep basins, fragmentation of subaerial lava flows that entered the sea and collapse of lava deltas and by flank collapse. About 16 debris-flow units (lithologic Unit VII) in Hole 953C range in thickness from $\sim 1$ to $50 \mathrm{~m}$. Most are composed of well-sorted massive lapillistone to coarse hyaloclastite tuff consisting of blocky, poorly vesicular shards, minor tachylite, and crystallized basalt. The top 5\%-10\% or so show laminar bedding to minor cross-bedding, the grain size rarely decreasing to fine sand (ash) size in the top beds. Basalt clasts up to $25 \mathrm{~cm}$ in diameter are common in the coarse-grained basal parts. Most particles in the stratigraphically highest deposits are vesicular to highly vesicular ash to lapilli-size clasts suggesting decreasing water depth. Coarse breccias at Hole 953C (lithologic Unit VI) consist of basalt clasts of diverse composition, angularity, and vesicularity, and some contain pillow rind fragments. Only the upper of three debrites at Site 956 (Cores 157-956B43R through 45R) and underlying turbidites consist dominantly of highly vesicular formerly glassy ash to lapilli-sized clasts. Lithic-rich debris-flow deposits at Site 956 (Cores 157-956B-45R through 48R, and 49R through 57R) consist chiefly of poorly vesicular, angular tachylite, crystallized basalt, and minor formerly glassy shards set in $\sim 30-50$ vol\% brown clay matrix.

About 300 very thin turbidite beds, $1-40 \mathrm{~cm}$ thick, deposited prior to the first ignimbrite-related ash deposit at Hole 953C are composed of variable amounts of dominantly silt- to sand-sized tachylitic and lesser amounts of vesicular to blocky altered shards and minor biogenic debris. They are interpreted to represent chiefly the subaerial growth stage of the basaltic shield and to have been derived dominantly from erosionally fragmented scoria and lava flows. The phenocryst assemblage in clasts and matrix of all deposits, mainly titanaugite and olivine $\left(\mathrm{Fo}_{83-88}\right)$ and minor plagioclase, changes with depth at both sites.

Most of the submarine basaltic clasts and clastic rocks from Hole $953 \mathrm{C}$ are more primitive mineralogically and chemically than the subaerial shield stage basalts. Ratios of incompatible trace elements are practically indistinguishable between mafic and moderately evolved rocks, between holes and between the bulk volcaniclastic rocks, basalt clasts, and subaerial rock shield basalts, suggesting that the source for the basalt magmas was fairly homogeneous during the late submarine and subaerial evolution. More evolved compositions (plagioclase-phyric hawaiites) are restricted to Site 956 coincident with the eruption of latestage evolved subaerial shield lavas in southwestern Gran Canaria. Fresh glass in the center of large lapilli in the uppermost of three debris flows (Core 157-956B-44R) is intermediate in composition, $\mathrm{MgO}$ ranging from 3.9 to $4.9 \mathrm{wt} \%$. Major and trace element concentrations of some 101 hyaloclastite bulk rocks and 20 clasts document major mobile element transfer. Basaltic glass is generally replaced by smectite. Zeolite phases, mostly phillipsite, and minor carbonate, are common pore-filling phases.

The vertical and lateral growth and changes in eruptive activity of the shield volcano are well reflected in the lithologic and compositional contrasts within and between the sections drilled at Hole 953C and Hole 956B (45 km southwest of Gran Canaria). Decreasing water depth of eruption is especially well documented in the increase in vesicularity in shards at Site 953 and extremely vesicular shards near the top of the basaltic section of Site 956. Eruptive activity had clearly decreased in the east of Gran Canaria during deposition of the late-stage turbidites at Hole 953C, as shown by the dominance of epiclastic particles in these rocks. Cores 157-956B-44R and 45R of the basaltic shield sequence at Hole 956B are considered to record younger active volcanism in western Gran Canaria that was taking place while largely epiclastic material was supplied to Site 953 in the northeast.

A recovered 3.75-m-thick interval consisting of layers of an unusual sandstone interbedded with turbidites of mixed volcaniclastic-biogenic lithology, on top of the basal 85-m-thick lithoclast-rich debrite in the lower part of Hole 956B (interval 157956B-48R-3, $23 \mathrm{~cm}$, to 49R-1, $26 \mathrm{~cm}$ ), consists dominantly of alkali amphibole, partially chloritized phlogopite and apatite, and minor Cr-spinel, sphene, and zircon mixed with foraminifers. We speculate that the huge basal debrite, underlying these sands and which we interpret as having been formed by collapse of the flanks of southwestern Gran Canaria, has caused major tsunamis that washed up beach sands on La Gomera, $\sim 115 \mathrm{~km}$ west of Gran Canaria.
\end{abstract}

\section{INTRODUCTION}

'Weaver, P.P.E., Schmincke, H.-U., Firth, J.V., and Duffield, W. (Eds.), 1998. Proc. ODP, Sci. Results, 157: College Station, TX (Ocean Drilling Program).

${ }^{2}$ GEOMAR Forschungszentrum, Wischhofstrasse 1-3, D-24148 Kiel, Federal Republic of Germany. hschmincke@geomar.de
The subaerial parts of oceanic islands resemble the tip of icebergs. Less than $10 \%$ of these huge volcanoes is above sea level. Most of the evolution of oceanic volcanoes thus takes place under water, and very 
little about this development is known. One of the major aims of drilling the volcanic apron of Gran Canaria during Leg 157 was to penetrate and recover deposits reflecting a significant part of the temporal, structural, volcanic, and compositional submarine evolution of the seamount that eventually became the island of Gran Canaria. Although drilling was not deep enough or close enough to the island to intersect and recover rocks representing the deep water stage, we now have excellent material to discuss a developmental stage in the growth of volcanic islands that is generally inaccessible for systematic scientific study.

Drilling of deposits representing the shield phase was successful. Hyaloclastites, lapillistones, and breccias make up the basal $293 \mathrm{~m}$ at Hole 953C, $68 \mathrm{~km}$ northeast (total penetration 1159 meters below seafloor [mbsf]), $38 \mathrm{~m}$ at Hole 954B, $34 \mathrm{~km}$ northwest (total penetration $446 \mathrm{mbsf}$ ), and $140 \mathrm{~m}$ at Hole $956 \mathrm{~B}, 45 \mathrm{~km}$ southwest (total penetration 704 mbsf) of the present shore of Gran Canaria (Figs. 1-3).

We are studying several interrelated problems posed by the basaltic fragmental rocks representing the submarine and subaerial shield stage: Do the deposits represent deep, moderate, or shallow water eruptive processes or transitions to the subaerial island shield stage? How many fragmentation processes can be distinguished from each other? What do the transport and depositional processes tell us about the constructional interval between submarine and subaerial shield phases of intraplate oceanic volcanoes? Do the primary mineralogical and chemical compositions of these rocks compared to those of the subaerial shield lavas represent different stages in the magmatic evolution of the volcano? Do the deposits from the northeastern and southwestern volcanic apron reflect different growth stages in the shield stage evolution of Gran Canaria? How much element transfer took place between the formerly dominantly glassy hyaloclastites and seawater?

Below, we call basaltic volcaniclastic deposits dominated by altered sideromelane shards hyaloclastite tuffs and, where the median exceeds $2 \mathrm{~mm}$, hyaloclastite lapillistones. Lithic-rich deposits are named basaltic siltstones, sandstones, or lapillistones and, where the median exceeds $64 \mathrm{~cm}$, lithic breccias. The upper stratigraphic boundary of the basaltic intervals is precisely defined by syn-ignimbritic tuff units representing the eruption of the widespread basaltrhyolite ignimbrite P1 on top of the basaltic shield of Gran Canaria (Freundt and Schmincke, 1992; 1995; Freundt and Schmincke, Chap. 14 , this volume). We refer to these here as P1 tuffs. Seismic constraints are insufficient to estimate the thickness of hyaloclastites below the bottom of the holes (Funck and Lykke-Anderson, Chap. 2, this volume). We speculate that at least several hundred meters of hyaloclastites occur at greater depths, because the deposits drilled are interpreted to represent the shallow to moderate water stage only. Deposits recording most of the evolution of the Gran Canaria seamount stage are still to be drilled.

We focus on the thick sections drilled at Sites 953 and 956 because they reflect the growth history of the island best. We treat the 38-m-thick basaltic breccia drilled at Hole 954B only briefly. At Hole 955A, P1 tuffs are underlain by $>30 \mathrm{~m}$ (565.68 mbsf to the bottom of the hole at $599.4 \mathrm{mbsf}$ ) of nannofossil clay-mixed sedimentary rocks, with minor basaltic detritus at the base of some turbidites, apparently representing eroded subaerial lavas. This area was probably outside the main pathways of basaltic volcaniclastic sediment transport dur-

\section{B}

$16^{\circ} \mathrm{W}$

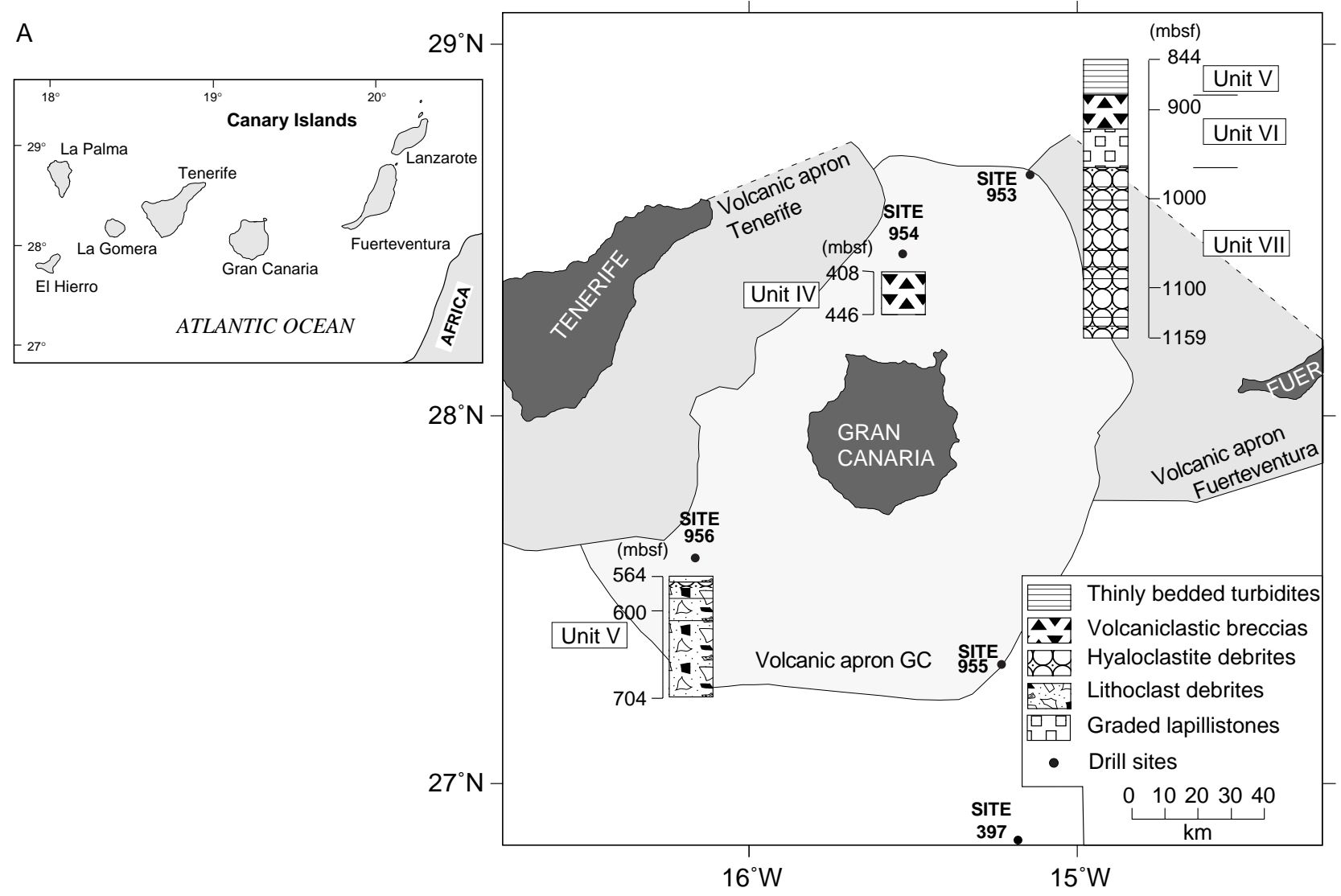

Figure 1. A. General map of the Canary Islands. B. Map of drill sites and simplified stratigraphic sections. 


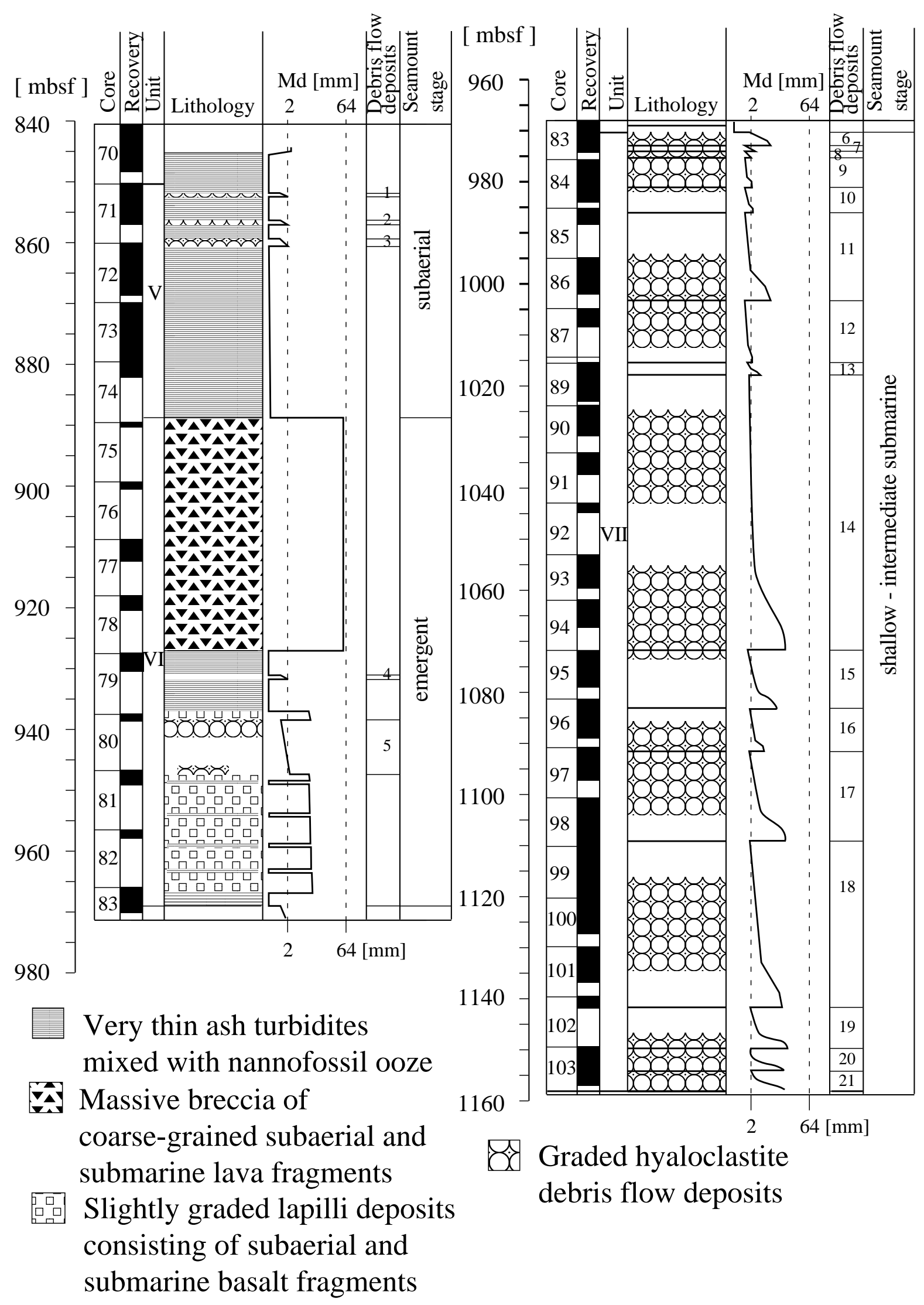

Figure 2. Stratigraphic section of Hole 953C from 844 to $1158.7 \mathrm{mbsf}$. 
$\square$ Nannofossil ooze

- Graded lithoclast debrites

38 Graded hyaloclastite debrites

Volcaniclastic sediment layers 560

: Exotic crystal sandstone

P1 syn-ignimbrite tephra
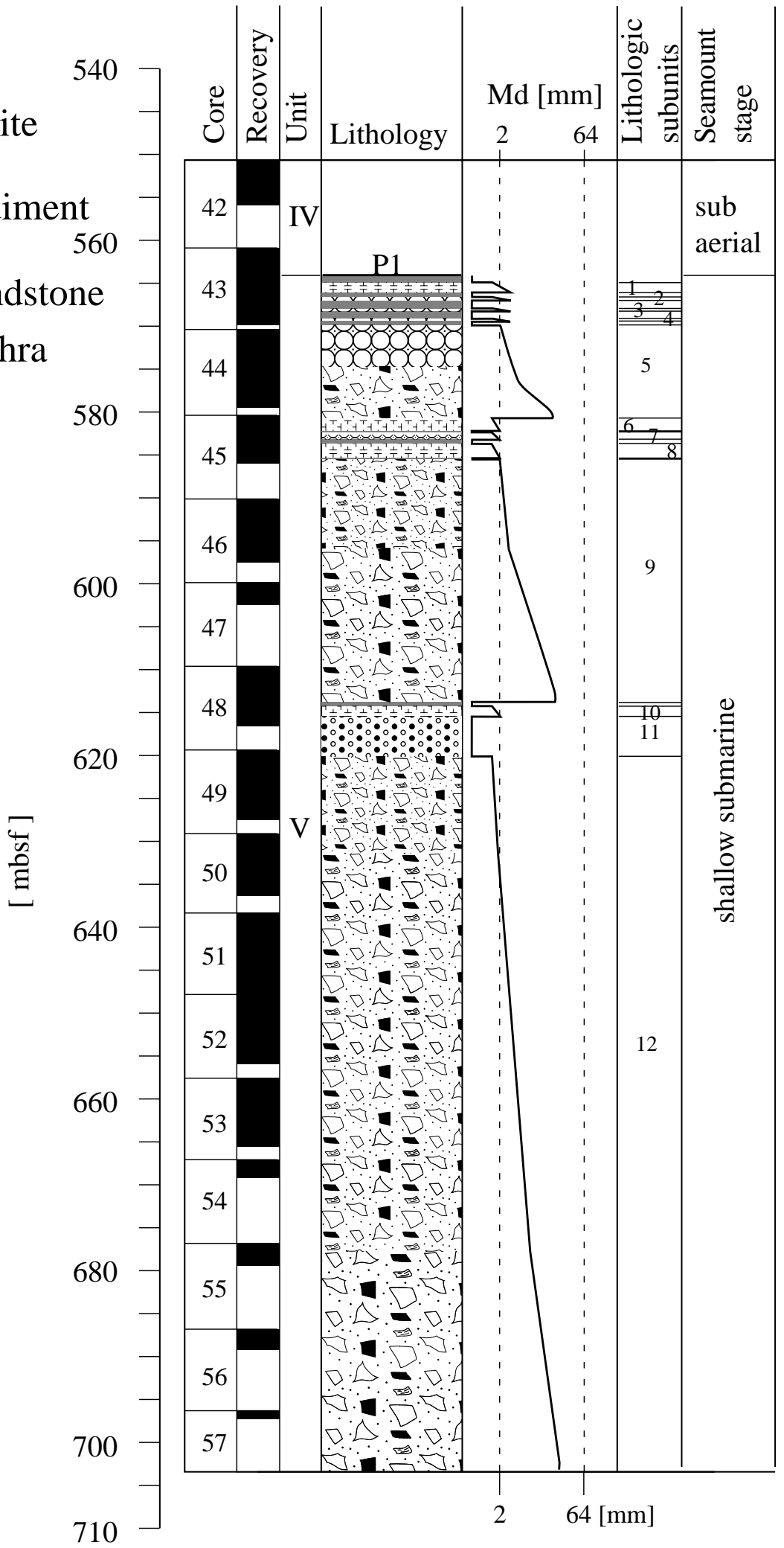

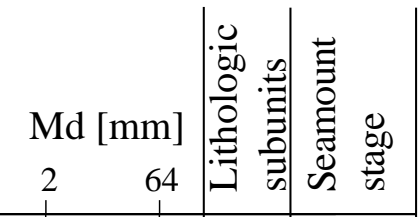

600

620 42 IV \begin{tabular}{|l|l|}
\hline 43 \\
\hline 44 \\
\hline
\end{tabular}
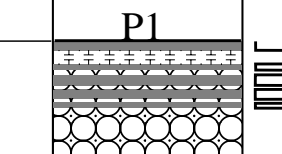

翌 ara $-\quad-\quad>$ \begin{tabular}{|l|l|}
\hline 45 & \\
\hline 46 & \\
\hline & \\
\hline
\end{tabular}

(1)

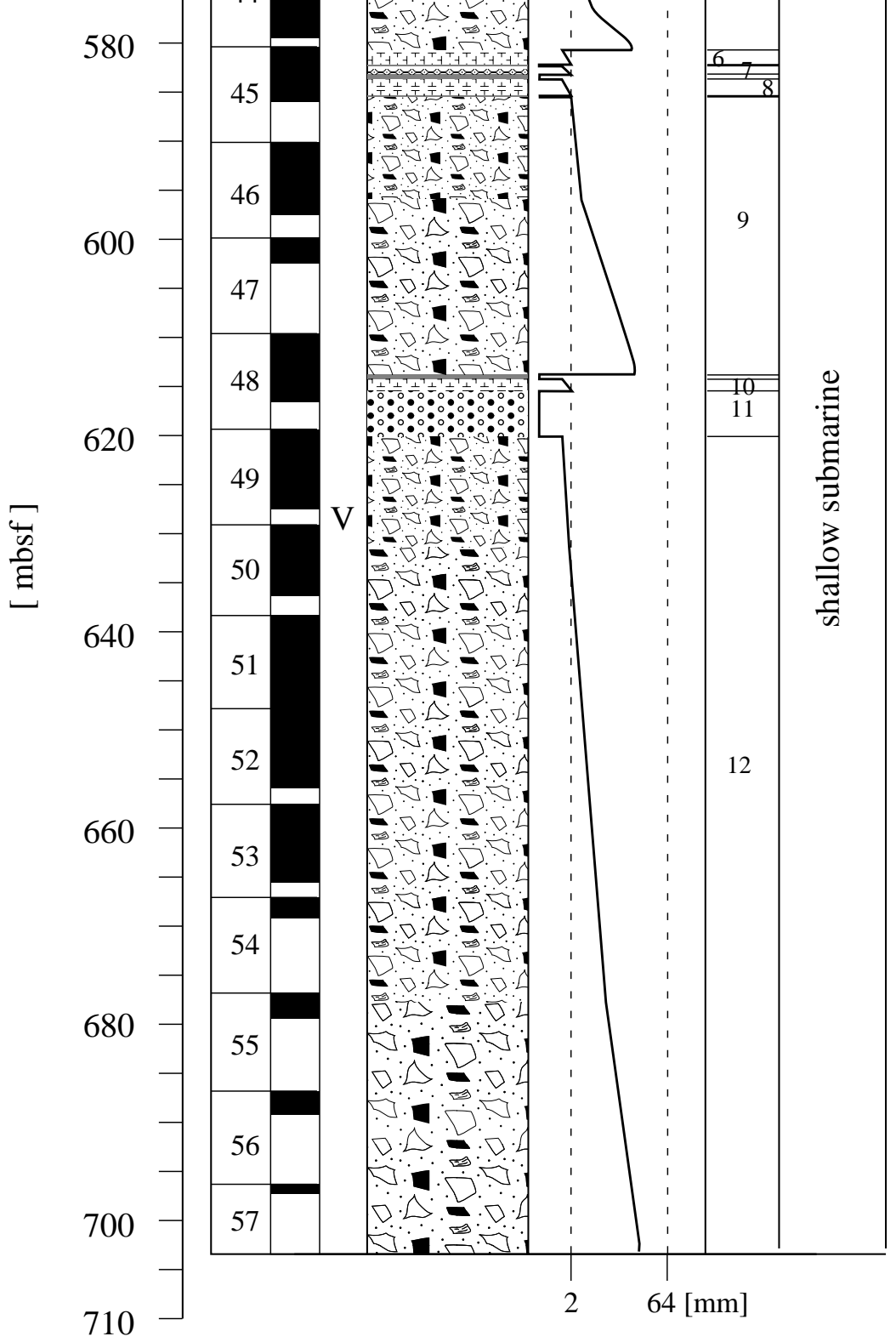
$\overrightarrow{7}$

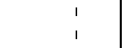

Figure 3. Stratigraphic section of Hole 956B from 564.1 to 704 mbsf. 
ing the subaerial shield stage and possibly the latter part of the shallow submarine stage. We do expect thicker hyaloclastite deposits at greater depth at this site, which is situated at the edge of the volcanic apron (Funck, 1996). Many interpretations in the present preliminary account are tentative.

\section{CLAST TYPES}

Schmincke and von Rad (1979) distinguished four types of clasts in the basaltic lapillistones drilled at Deep Sea Drilling Project (DSDP) Site 397, $50 \mathrm{~km}$ southeast of Site 955: tachylite, (altered) sideromelane, single crystals and crystallized basalt. We are adding two more categories based on the microscopic study of 150 thin sections from Holes 953C, 954B, 955A, and 956B: basalt clasts $>6.4 \mathrm{~cm}$ in diameter and drop-shaped glassy to formerly glassy lapilli. We find it convenient to separately discuss particles $<$ and $>2 \mathrm{~cm}$. Volume percentages of major clast types in Holes $953 \mathrm{C}$ and $956 \mathrm{~B}$ are shown in Figures 4 and 5.

\section{Clasts $<2 \mathrm{~cm}$ in Diameter}

\section{Sideromelane}

We distinguish two major types of mafic clasts that formerly consisted of sideromelane but are now generally altered to smectite (Pl. 1, Figs. 1-4). Most common are angular, blocky, poorly vesicular shards with $<20$ vol\% vesicles. The second type is represented by vesicular ash to lapilli-sized fragments whose vesicularity may exceed $50 \mathrm{vol} \%$ (Pl. 2, Fig. 5) and which are interpreted to have formed during explosive eruptions in shallow water. Extremely vesicular, pumiceous shards that occur in turbidites near the top of the basalt section at Site 956 and as xenoliths in the lower rhyolitic ash turbidites may record extremely shallow-water explosive eruptions, possibly emergent tuff rings. Staudigel and Schmincke (1984), who studied an uplifted, submarine, compositionally similar alkali basalt series on La Palma, estimate the minimum depth for explosive eruptions at $\sim 700$ meters below sea level. This estimate is poorly constrained, but is accepted here in lieu of a more precise barometric method. We cannot exclude greater water depths of eruption nor generation of poorly vesicular blocky shards by degassed subaerial lavas that entered the sea along the shore. All transitions from nonvesicular to highly vesicular may occur within the same thin section. Nevertheless, at both holes (953C and 956B), the highly vesicular shards dominate the upper part of the sections, the nonvesicular in the lower (Pl. 1, Figs. 1-4).

\section{Drop-Shaped Lapilli}

Glassy and formerly glassy, commonly nonvesicular to poorly vesicular lapilli with rounded outlines and subspherical to drop-like shapes are common in the 10-m-thick debrite in Hole 956B (Subunit 5; Cores 157-956B-44R and 45R) and also occur sporadically in the thick basal debrite in Hole 956 B (Figs. 6, 7). We tentatively interpret these clasts as formed by the draining of subaerial/submarine lava tubes, especially where subaerial lava flows entered the sea, but we cannot exclude an origin by submarine lava fountains.

\section{Tachylite}

We distinguish two major end-members of tachylite types from each other: tachylitic shards, commonly vesicular, which represent entire or broken lapilli/scoria, and tachylitic rock fragments, which are nonvesicular and angular or subrounded, representing fragments of quickly cooled basalt (Pl. 2, Figs. 1-4). The latter are most com- mon in the turbidites interpreted to have formed during the emergent stage of eastern Gran Canaria (Unit V at Hole 953C and also in Cores 157-956B-43R and 44R; Pl. 2, Fig.1) and in the lithic-rich basal debrite at the base of Hole 956B interpreted as having resulted from subaerial/submarine flank collapse. Some smaller clasts are orange (oxidized), suggesting subaerial exposure.

\section{Crystallized Basalt}

Generally angular to subrounded, rarely rounded, crystalline basalt clasts consist dominantly of groundmass plagioclase, augite, and $\mathrm{Fe} / \mathrm{Ti}$-oxides, and, at lower stratigraphic levels, also olivine (Pl. 3, Figs. 1-4). Phenocrysts are mainly clinopyroxene, mostly altered olivine and minor plagioclase. There are all gradations in degree of crystallinity from microcrystalline to doleritic, textures ranging from hyalopilitic to subophitic, intergranular to intersertal being most common.

\section{Single Crystals}

We count clasts as single crystals when a particle consists largely or entirely of a phenocryst phase. Single crystals are overwhelmingly titanaugite and less common olivine and plagioclase (Pl. 2, Fig. 4; Figs. 8-10).

\section{Clasts $>2 \mathrm{~cm}$ in Diameter}

Lithic accessory clasts are dominantly poorly, rarely. moderately to highly vesicular and range from tachylitic to fine- to mediumgrained crystalline basalt (Pl. 4, Figs. 3, 4). Most clasts are subangular to subrounded and range up to $22 \mathrm{~cm}$ in diameter based on their length in the cores, where they are concentrated in the basal parts of debris-flow deposits. Most clasts are clinopyroxene-olivine-phyric. Minor aphyric and plagioclase-phyric basalts are especially common in the breccia from Hole 954B. Some clasts near the base of Unit VII (Hole 953C) are picritic with abundant large olivine pseudomorphs or, rarely, fresh crystals (Fig.10), and clinopyroxene.

Except for the basaltic siltstone/sandstone turbidites of Unit V (Hole 953C) and Subunit 1-4 (Hole 956B), in which the different clasts types occur in highly varying proportion, a clear grouping in the ternary diagram suggested by Schmincke and von Rad (1979) into lithic-dominated and sideromelane-dominated rocks exists for almost all deposits. The breccias of Hole 954B and the lithic-dominated basal debris-flow unit at Hole 956B contain clasts of vesicular basalt and red oxidized scoriaceous fragments indicating derivation from subaerial basalts. However, these units also contain minor altered, vesicular hyaloclastite grains, suggesting that products of both submarine and subaerial volcanism were mixed together prior to deposition of the coarse clastic units. The good rounding of some basalt clasts indicates abrasion and reworking of subaerial volcanic rocks.

\section{Matrix and Cement}

The matrix of debris-flow deposits consists of green to brown noncalcareous to nannofossil-bearing clay (Hole 956B) and minor zeolites (Pl. 2, Fig. 4; Pl. 4, Figs. 1-4) and grayish white calcareous clay (954B), fine grained fragments of basalt, altered shards, crystals of clinopyroxene, altered olivine, minor plagioclase, and zeolites. In finer grained deposits, for example, the turbiditic sandstones and siltstones at the top of the successions that consist largely of epiclastic particles, it is commonly difficult to distinguish true clastic matrix from altered fine-grained glass shards as was the case with the Site 397 volcaniclastic rocks (Schmincke and von Rad, 1979). Most of the 


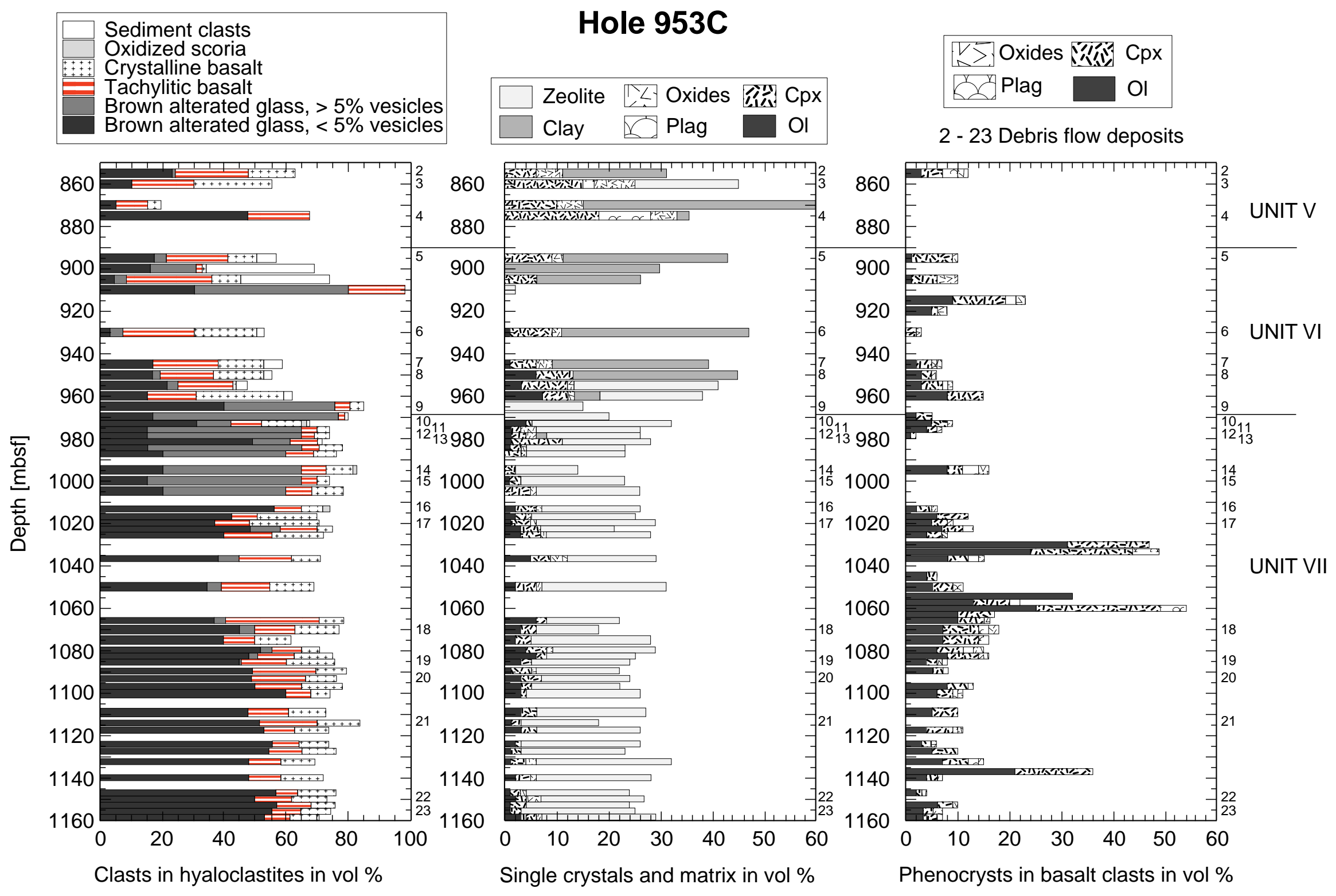

Figure 4. Clast and phenocryst distribution vs. depth of Hole 953C. Note that the numbers of the y-axis indicate the depth of every second sample. 


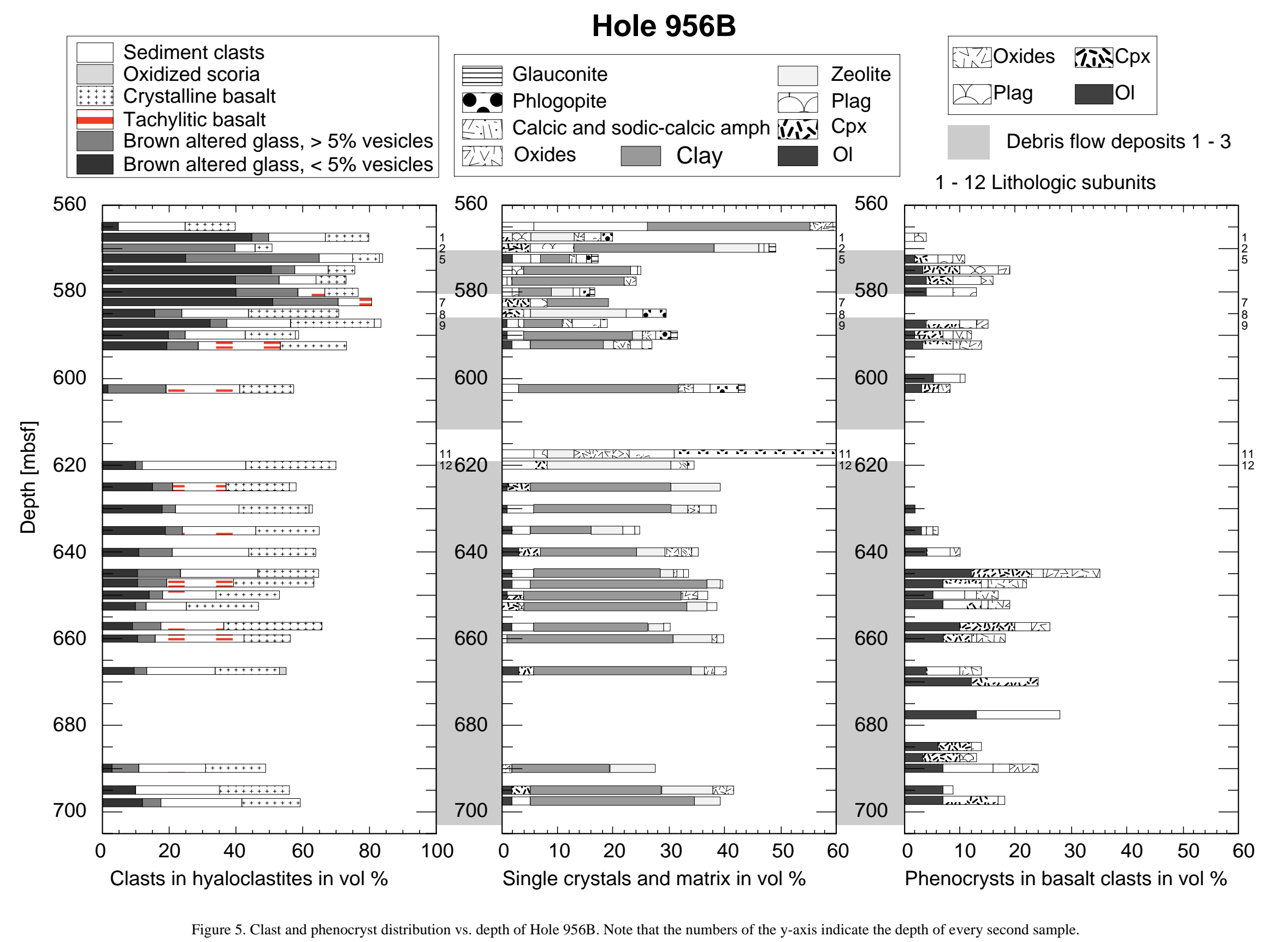




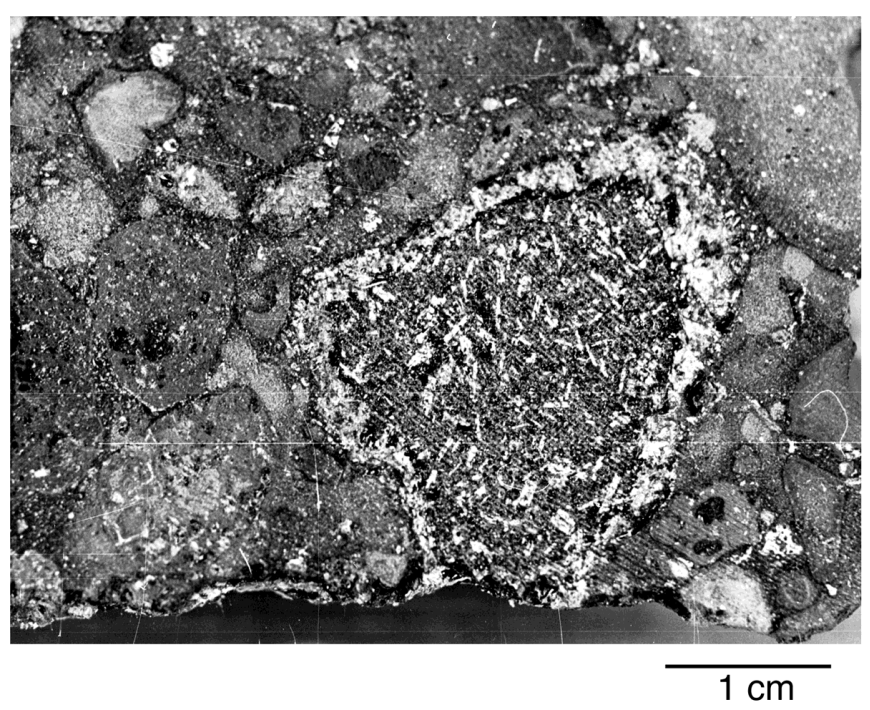

Figure 6. Lapillistone in the uppermost of three debris flows at Hole 956B. Note dark colored 3-cm lapillos with plagioclast phenocrysts and lightcolored altered rim in lower right (Sample 157-956B-44R-5, 43-50 cm).

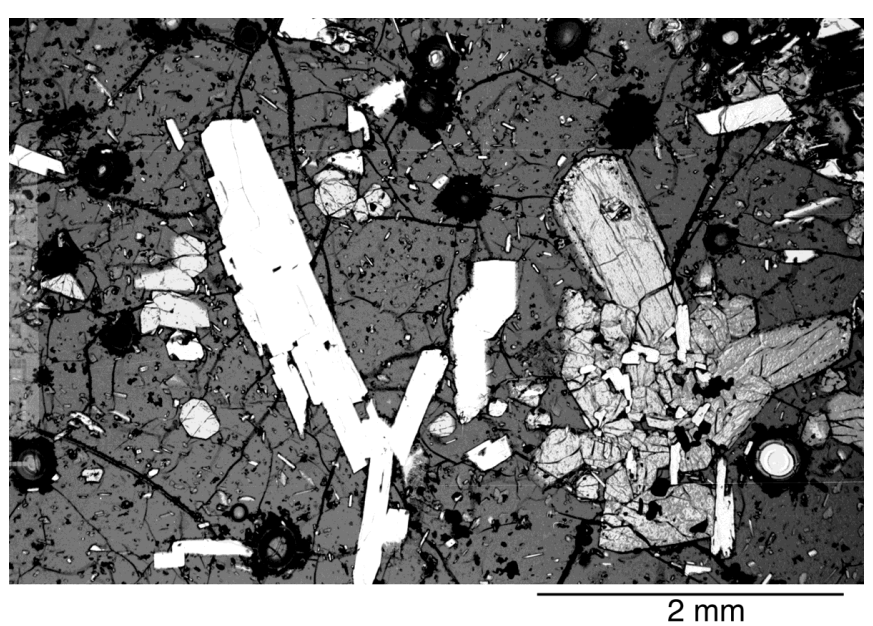

Figure 7. Plagioclase and clinopyroxene phenocrysts and glomerocrysts surrounded by fresh glass of lapillos from Hole 956B (Sample 157-956B-44R-4, $27-42 \mathrm{~cm})$.

hyaloclastite tuffs and lapillistones at Hole 953C (Units VI and VII) lack a clastic matrix (Pl. 1, Figs. 1, 2). The zeolite cement in the deposits of the units is chiefly phillipsite, whereas smaller amounts of green clay in these hyaloclastites are thought to be authigenic rather than clastic (Figs. 11, 12).

\section{XRF AND ELECTRON MICROPROBE ANALYTICAL METHODS}

Major and trace element concentrations were determined for 100 bulk rocks, including 20 clasts by X-ray fluorescence (XRF; Philips $\mathrm{X}^{\prime}$ Unique). Twenty powders of bulk rocks and clasts done aboard ship were reanalyzed. For Hole 953C, both powder and glass pellets, for Holes 954B and 956B, only glass pellets were prepared. XRF analyses were carried out on melt (glass) pellets using a Philips PW

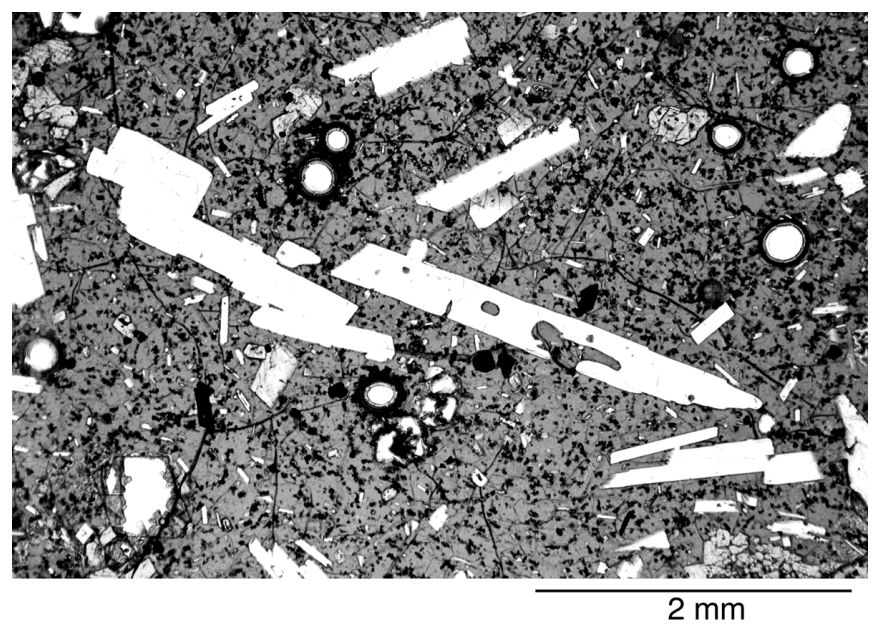

Figure 8. Plagioclase phenocrysts with melt inclusions and minor clinopyroxene and microphenocrysts set in fresh glassy lapillos with more pronounced alteration around vesicles (Sample 157-956B-44R-5, 70-82 cm).

1400 and a Philips PW 1480 spectrometer. Standard OXIQUANT software was used. Two-hundred thirty international reference samples and 41 synthetic standards were employed. The pellets were produced from melting of rock powder at $1000^{\circ} \mathrm{C}$ for $\sim 10$ min with an added flux of lithium metaborate and dilithium tetraborate (Merck A $12)$ at a mixing ratio of $1: 4$. The melt was cooled in a mold $34 \mathrm{~mm}$ in diameter. $\mathrm{H}_{2} \mathrm{O}$ was measured by closed-system coulombmetric titration. Water degassing from rock powder heated at $1300^{\circ} \mathrm{C}$ in a Ptcrucible placed in an induction furnace was carried in a nitrogen gas stream and analyzed in Karl Fischer reagent. Sulfur, carbon, and $\mathrm{CO}_{2}$ were analyzed by infrared photometry using a Rosemount CWA 5003 for $\mathrm{CO}_{2}$ and a Rosemount CSA 5003 for carbon and sulfur. Trace elements determined include $\mathrm{Cr}, \mathrm{Ni}, \mathrm{V}, \mathrm{Cu}, \mathrm{Zr}, \mathrm{Nb}, \mathrm{Ce}, \mathrm{Y}, \mathrm{Rb}$, $\mathrm{Zn}, \mathrm{Ba}$, and Sr. Representative analyses are listed in Tables 1-6.

Major elements and $\mathrm{S}, \mathrm{Cl}$, and $\mathrm{F}$ were analyzed with a Cameca SX-50 electron probe at GEOMAR. For major elements, analytical conditions were $10 \mathrm{kV}$ of accelerating voltage, $10 \mathrm{nA}$ of beam current, and $10 \mathrm{~s}$ of peak counting time. Analyses were performed with an electron beam rastered to $10 \times 12 \mu \mathrm{m}$. Cameca synthetic oxides, basaltic glasses USNM 111240/52 and USNM 113498/1 (VG-A99), apatite USNM 104021, microcline USNM 143966 (Jarosewich et al., 1980), and spinel Yb-126 were used as standards for calibration. S, $\mathrm{F}$, and $\mathrm{Cl}$ were measured as trace elements with accelerating voltage of $15 \mathrm{kV}$ and beam current of $20-25 \mathrm{nA}$. The electron beam was rastered to $10 \times 12 \mu \mathrm{m}$, and peak counting times were $30 \mathrm{~s}$. Standards used were chalcopyrite for S, scapolite USNM R6600-1 for Cl, and Durango fluorapatite USNM 104021 for F, respectively (Jarosewich et al., 1980). As monitor sample for S, we used basaltic ALV981R23 (Metrich and Clocchiatti, 1989), for $\mathrm{F}$ and $\mathrm{Cl}$ comenditic glass KN18 (Mosbah et al., 1991). Four to ten spots were measured within fresh sideromelane. The detection level was estimated to be $0.01 \mathrm{wt} \%$ with uncertainties of $5 \%-15 \%$ relative for volatile concentrations $>0.05$ $\mathrm{wt} \%$, and up to $20 \%-40 \%$ relative for the concentrations $<0.05 \mathrm{wt} \%$.

\section{MINERALOGY}

The phenocryst assemblage-mainly clinopyroxene (augite) and olivine $\left(\mathrm{Fo}_{83-88}\right)$, but also minor plagioclase-of the basalt clasts and volcaniclastic rocks compositionally resembles that of the subaerial shield basalts and is typical of alkali basalts (Figs. 13, 14; Tables 713). Fresh olivine occurs in several basalt clasts and some large al- 


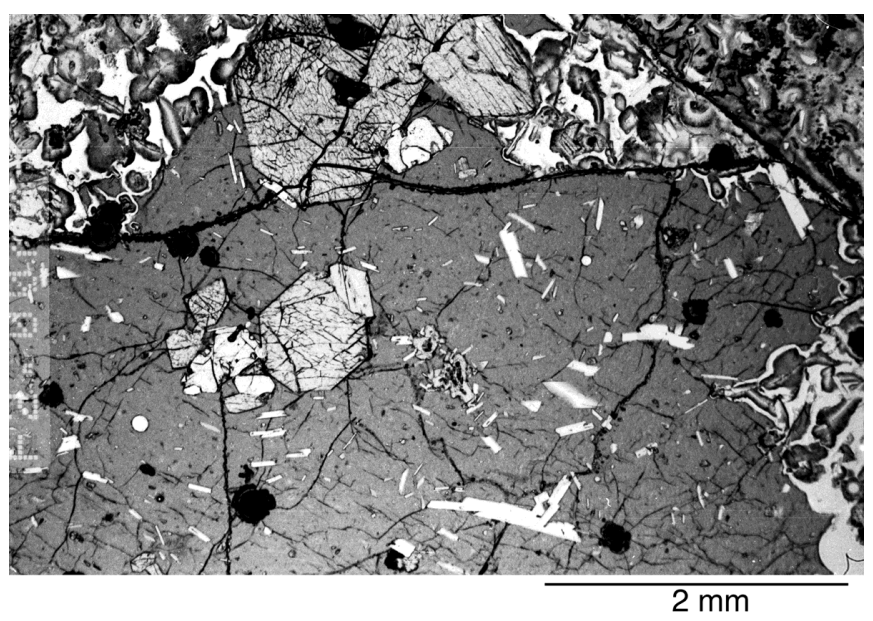

Figure 9. Fresh interior of glassy lapillos with clinopyroxene and feldspar phenocrysts and microphenocrysts showing transition to altered rim (Sample 157-956B-44R-4, 110-124 cm).

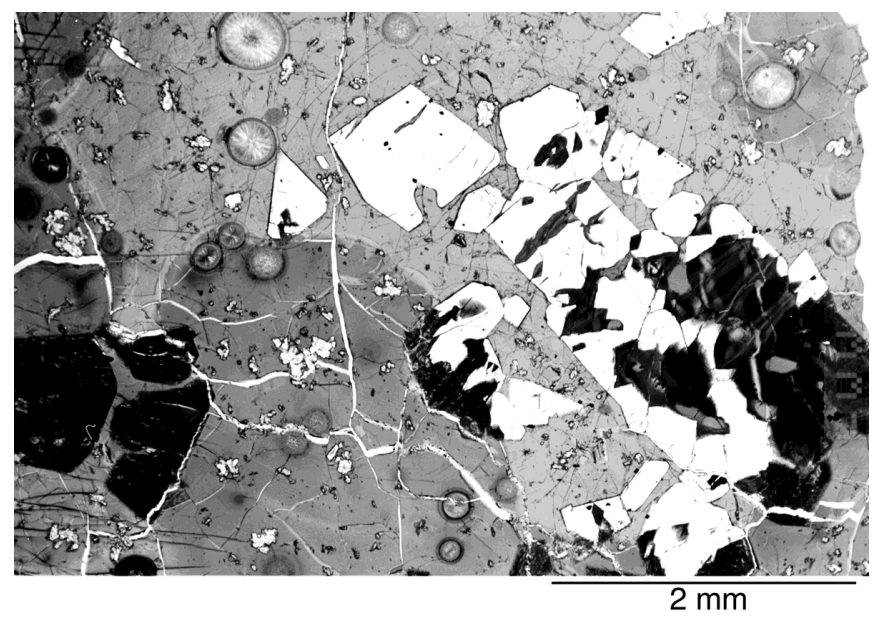

Figure 10. Partially altered olivine phenocrysts and smektite altered sideromelane lapillos and vesicles filled with layer silicates (Sample 157-953C94R-3, 125-146 cm).

tered sideromelane clasts. Clinopyroxene phenocrysts and glomerocrysts occur in crystallized, tachylitic and formerly glassy shards and rock fragments. Large subhedral clinopyroxene crystals are common as isolated crystals. Clinopyroxene glomerocrysts are especially common in lithologic Units V and VI of Hole 953C and the upper 20 $\mathrm{m}$ of Hole 956B (Figs. 7, 9). Plagioclase phenocrysts (Fig. 7) are only abundant in the upper debris-flow deposit of Hole 956B (Cores 157956B-44R and 45R) and are up to $1.5 \mathrm{~mm}$ long (Fig. 8). At Hole 953C, minor plagioclase microphenocrysts are restricted to Unit V.

\section{Mineralogy of the "Placer Sands" of Hole 956B}

A very unusual subunit of dominantly light-colored bedded to cross-bedded sandstones, making up $\sim 2 \mathrm{~m}$ of a 5-m interval of nannofossil/foraminifer ooze turbidites, occurs between 612.5 and $619.68 \mathrm{mbsf}$ at Hole 956B (interval 157-956B-48R-3, $123 \mathrm{~cm}$, to 49R-1, $26 \mathrm{~cm}$ ). It consists of parallel- and cross-laminated foraminifer-crystal sandstone composed of alkali amphibole, apatite, largely chloritized phlogopite, and lesser amounts of green clinopyroxene, zircon, Cr-spinel, and sphene.

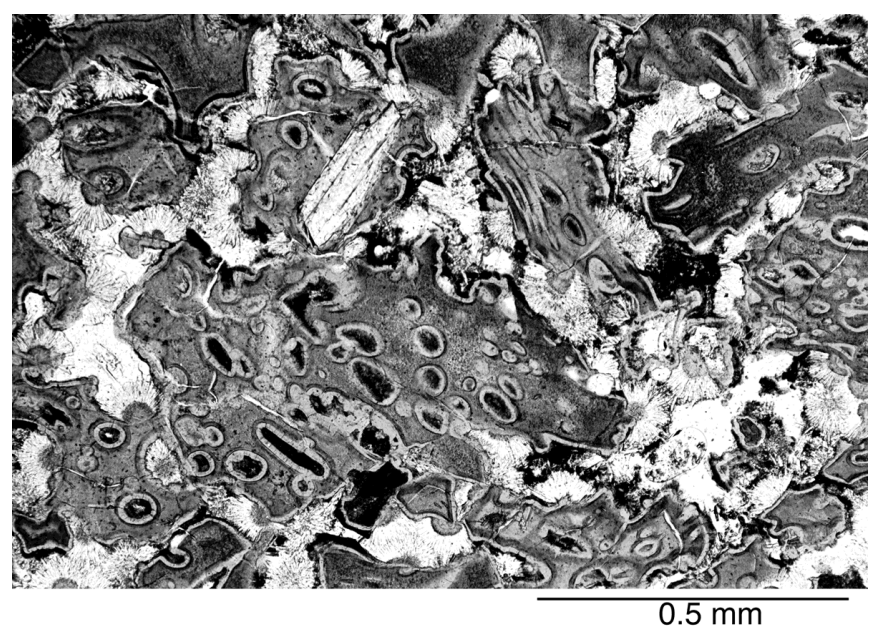

Figure 11. Sideromelane clasts of intermediate vesicularity altered to layer silicate. Pore space between well-sorted lapilli filled with phillipsite (Sample 157-953C-83R-6, 107-120 cm).

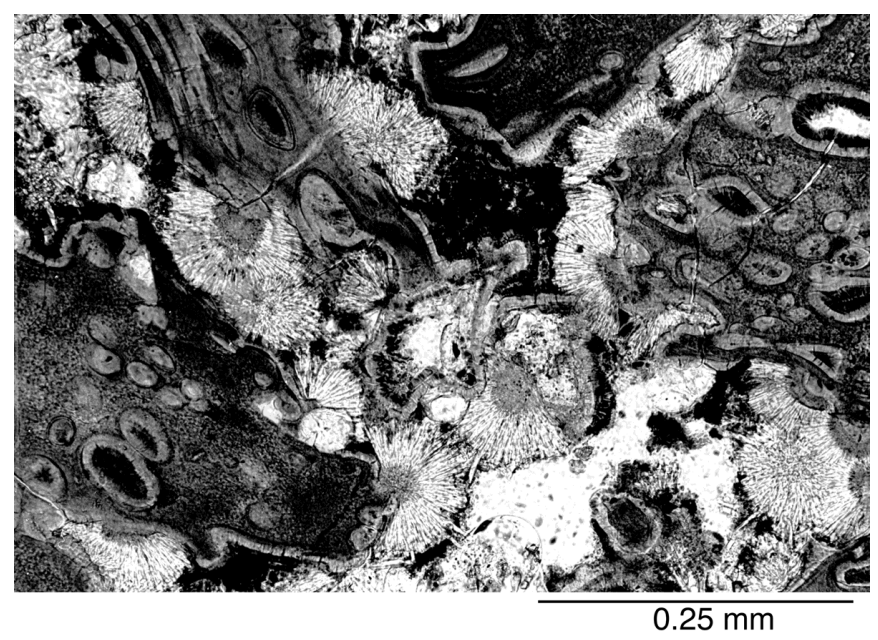

Figure 12. Detail of Figure 11 showing marginal replacement of smectite altered sideromelane by phillipsite (Sample 157-953C-83R-6, 107-120 cm).

Amphibole crystals (Table 14) are generally 1-2 mm long and euhedral with absorption colors varying from yellow and reddish yellow to brownish, and are highly concentrated in the "placer sand layers" where they can make up as much as $50 \mathrm{vol} \%$ of some dark layers (Fig. 15). Compositionally, they are alkali amphiboles with low $\mathrm{Ca}$ and $\mathrm{Al}$ and high $\mathrm{Na}, \mathrm{K}$, and $\mathrm{Mg}$ concentrations (Fig. 16). They are classified as edenitic hornblende, edenite, magnesiohornblende and magnesiokatophorite (Fig. 17). Mica crystals (Table 15), generally up to $2 \mathrm{~mm}$ long, are phlogopites with $\mathrm{Mg}$ concentration ranging from 20.9 to $22.3 \mathrm{wt} \%$ (Fig. 18). They are highly concentrated in the placer sandstones (Hole 956B), but are sporadically present throughout the overlying basaltic volcaniclastics at Hole 956B and continue as "vagabond xenocrysts" into the tephra units of the Lower Mogán at Hole 956B (Sumita and Schmincke, Chap. 15, this volume).

\section{Alteration}

Clinopyroxene is stable even in the most altered rocks. Fresh melt inclusions are not uncommon in the pyroxenes and have been studied in more detail by Gurenko et al. (Chap. 22, this volume) and Wallace 
Figure 13. Clinopyroxene composition of Holes 953C, 954B, and 956B. Various oxides plotted vs. $\mathrm{CaO}(\mathrm{wt} \%)$. EMS data used in the plots are shown in Tables 1, 3, and 6 .
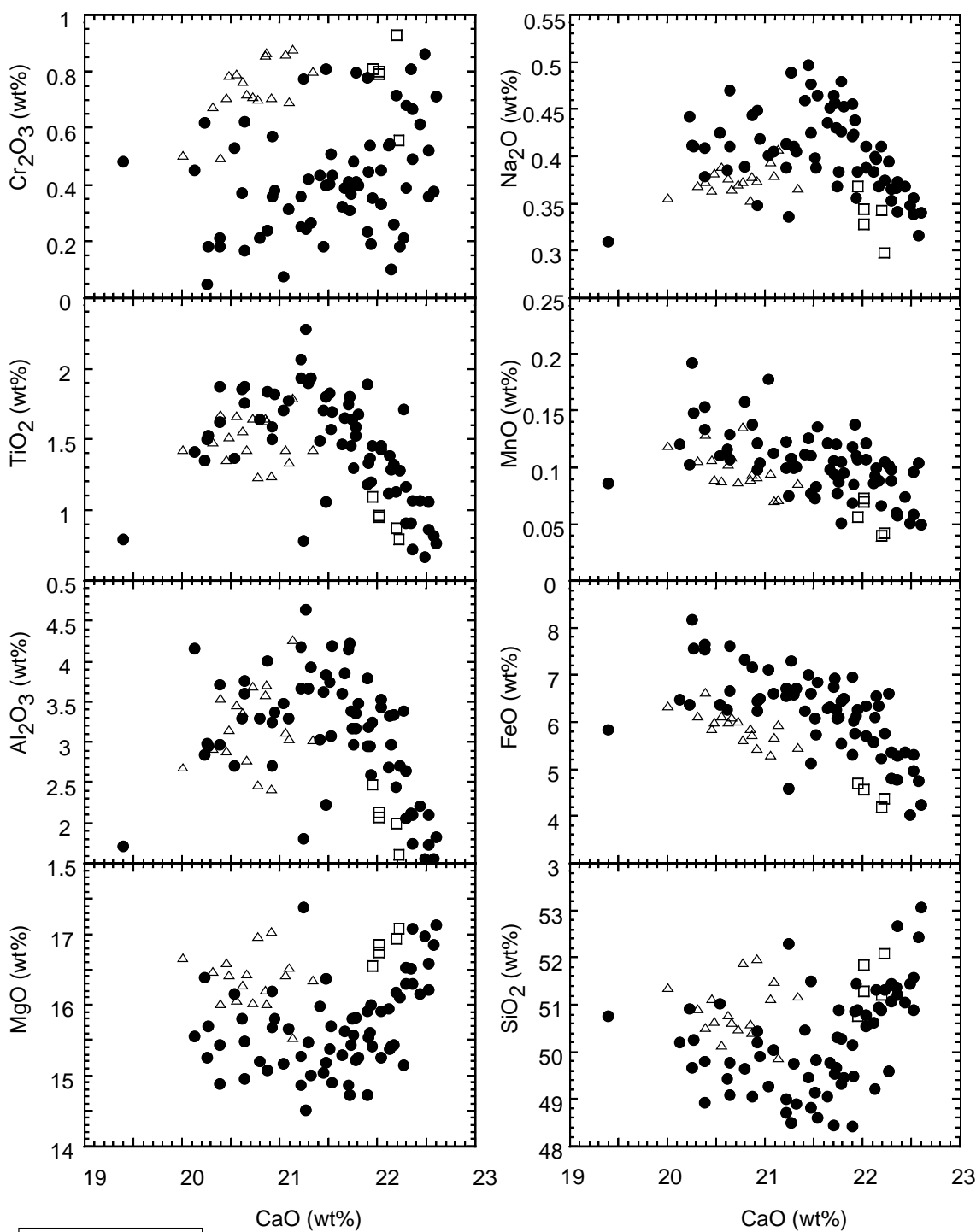

Hole 956B Hole 954B Hole 953C

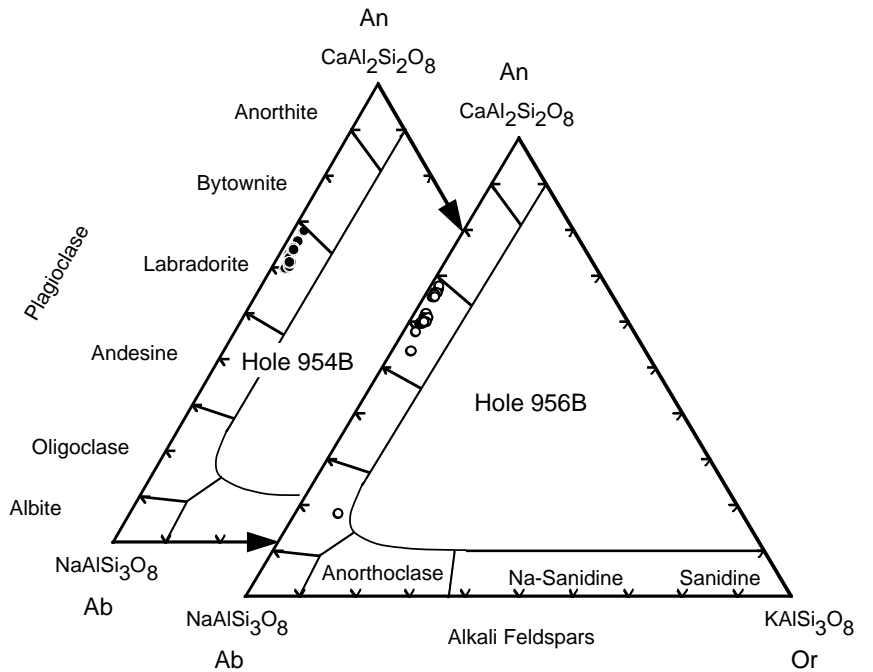

Figure 14. Left: Plagioclase composition of Hole 954B. Right: Plagioclase composition of Hole 956B.
(Chap. 24, this volume). Plagioclase ranges from fresh to altered. Olivine alteration products are mostly sheet silicates, but carbonates and iddingsite also occur. Basaltic glass (sideromelane), the dominant primary material in the hyaloclastites, is generally replaced by smectite. A range of zeolite phases, mostly phillipsite (Fig. 11), and minor analcime, natrolite, gmelinite, and carbonate occur in the interstices. Alteration was chiefly diagenetic, but some hydrothermal effects are reflected in higher temperature phases prehnite and apophyllite (lower hyaloclastites of Unit VII at Hole 953C) and laumontite in Cores 157-956B-44R through 46R (Schmincke, Weaver, Firth, et. al., 1995). Some smectite-altered shards have been replaced by phillipsite at their margins (Fig. 12).

\section{GEOCHEMISTRY}

Both coarse (sand-sized) and fine-grained (silty) sediments were analyzed. The samples include relatively pure tuffs as well as sandstones composed of volcanic rock clasts and hyaloclastites completely altered to clay and zeolites. The major element data used in the figures were recalculated $\mathrm{H}_{2} \mathrm{O}$ - and $\mathrm{CO}_{2}$-free. Major and trace element concentrations are compared to 30 analyzed subaerial basalts in Fig- 
Table 1. XRF major and trace element analyses of whole rock samples, Hole 953C.

\begin{tabular}{|c|c|c|c|c|c|c|c|c|c|c|c|c|}
\hline Hole, core, section: & $\begin{array}{c}953 \mathrm{C}-73 \mathrm{R}-4 \\
\end{array}$ & 953C-82R-1 & 953C-83R-3 & $953 \mathrm{C}-83 \mathrm{R}-3$ & 953C-83R-4 & 953C-83R-5 & 953C-83R-5 & 953C-83R-6 & 953C-84R-4 & 953C-84R-6 & $953 \mathrm{C}-84 \mathrm{R}-7$ & 953C-84R-8 \\
\hline Interval $(\mathrm{cm})$ : & 25-18 & $42-44$ & 13-24 & 94-108 & 41-45 & $0-23$ & 119-121 & 107-120 & 24-42 & 88-104 & $47-50$ & $27-44$ \\
\hline Depth (mbsf): & 874.23 & 956.82 & 968.44 & 969.25 & 970.15 & 970.88 & 972.07 & 973.21 & 979.21 & 982.31 & 982.95 & 984.11 \\
\hline Lithologic unit: & $\mathrm{V}$ & VI & VI & VII & VII & VII & VII & VII & VII & VII & VII & VII \\
\hline \multicolumn{13}{|l|}{ Major elements (wt\%) } \\
\hline $\mathrm{SiO}_{2}$ & 44.23 & $\begin{array}{r}41.55 \\
390\end{array}$ & $\begin{array}{r}43.61 \\
328\end{array}$ & 43.64 & 43.03 & 43.70 & 43.13 & 42.83 & 44.10 & 43.47 & 42.94 & 43.23 \\
\hline $\mathrm{TiO}_{2}^{2}$ & 2.78 & $\begin{array}{l}3.90 \\
8.05\end{array}$ & 3.28 & 3.25 & 3.06 & 2.83 & 2.88 & 3.41 & 2.95 & 3.37 & 3.36 & 3.36 \\
\hline $\begin{array}{l}\mathrm{Al}_{2} \mathrm{O}_{3} \\
\mathrm{Fe} * *\end{array}$ & 11.41 & $\begin{array}{r}8.95 \\
12.54\end{array}$ & 10.05 & & $\begin{array}{r}9.36 \\
1136\end{array}$ & 8.78 & 8.72 & 9.98 & 8.57 & 9.76 & 9.51 & 9.52 \\
\hline $\mathrm{FeO}^{*}$ & 11.04 & 12.54 & 10.83 & 10.85 & 11.36 & 11.54 & 11.33 & 11.63 & 11.55 & 11.18 & 11.63 & 11.12 \\
\hline $\mathrm{MnO}$ & 0.18 & 0.18 & 0.19 & 0.16 & 0.17 & 0.17 & 0.17 & 0.18 & 0.17 & 0.17 & 0.18 & 0.17 \\
\hline $\mathrm{MgO}$ & 9.22 & 13.13 & 13.22 & 13.81 & 14.84 & 16.95 & 14.81 & 10.32 & 13.76 & 10.09 & 10.77 & 11.42 \\
\hline $\mathrm{CaO}$ & 6.79 & 8.83 & 5.23 & 5.36 & 5.30 & 5.34 & 6.67 & 7.51 & 7.82 & 8.23 & 7.73 & 7.77 \\
\hline $\mathrm{Na}_{2} \mathrm{O}$ & 4.00 & 2.82 & 3.50 & 3.50 & 2.96 & 2.83 & 2.40 & 3.36 & 2.57 & 3.40 & 3.10 & 3.12 \\
\hline $\mathrm{K}_{2} \mathrm{O}$ & 1.13 & 0.40 & 1.18 & 0.96 & 0.53 & 0.21 & 0.37 & 1.29 & 0.76 & 1.23 & 0.86 & 1.11 \\
\hline $\mathrm{P}_{2} \mathrm{O}_{5}$ & 0.38 & 0.51 & 0.43 & 0.43 & 0.40 & 0.37 & 0.39 & 0.44 & 0.39 & 0.41 & 0.44 & 0.45 \\
\hline LOI & 6.60 & 5.27 & 6.71 & 6.49 & 6.55 & 4.93 & 6.83 & 6.60 & 5.88 & 6.20 & 8.06 & 6.38 \\
\hline Total & 97.76 & 98.08 & 98.23 & 98.22 & 97.56 & 97.65 & 97.70 & 97.55 & 98.52 & 97.51 & 98.58 & 97.65 \\
\hline \multicolumn{13}{|l|}{ Trace elements (ppm) } \\
\hline $\mathrm{V}$ & 244 & 240 & 248 & 253 & 209 & 234 & 197 & 245 & 258 & 274 & 231 & 297 \\
\hline $\mathrm{Cr}$ & 883 & 771 & 557 & 541 & 749 & 1055 & 873 & 765 & 1028 & 873 & 851 & 828 \\
\hline Co & 88 & 81 & 58 & 49 & 55 & 61 & 87 & 56 & 69 & 64 & 56 & 76 \\
\hline $\mathrm{Ni}$ & 343 & 447 & 328 & 365 & 498 & 645 & 561 & 403 & 563 & 336 & 412 & 405 \\
\hline $\mathrm{Cu}$ & 107 & 101 & 162 & 128 & 98 & 132 & 96 & 105 & 98 & 133 & 105 & 141 \\
\hline $\mathrm{Zn}$ & 114 & 115 & 111 & 100 & 98 & 117 & 107 & 122 & 111 & 104 & 109 & 102 \\
\hline $\mathrm{Rb}$ & 18 & 0 & 38 & 29 & 17 & $<4$ & 7 & 34 & 16 & 25 & 21 & 29 \\
\hline $\mathrm{Sr}$ & 289 & 361 & 400 & 401 & 396 & 364 & 443 & 447 & 385 & 550 & 546 & 463 \\
\hline $\mathrm{Y}$ & 24 & 28 & 28 & 30 & 23 & 24 & 24 & 31 & 24 & 27 & 27 & 29 \\
\hline $\mathrm{Nb}$ & 35 & 54 & 33 & 38 & 34 & 34 & 35 & 38 & 34 & 36 & 38 & 40 \\
\hline $\mathrm{Ba}$ & 526 & 148 & 515 & 376 & 196 & 45 & 90 & 259 & 91 & 198 & 122 & 193 \\
\hline $\mathrm{Ce}$ & 69 & 102 & 56 & 47 & 69 & 48 & 61 & 46 & 53 & 49 & 52 & 63 \\
\hline $\mathrm{Zr}$ & 184 & 318 & 250 & 244 & 227 & 209 & 212 & 257 & 222 & 231 & 234 & 249 \\
\hline $\mathrm{Nd}$ & 31 & 46 & 33 & 22 & 26 & 25 & 27 & 27 & 25 & 32 & 24 & 32 \\
\hline $\mathrm{Zr} / \mathrm{Nb}$ & 5.26 & 5.89 & 7.58 & 6.42 & 6.68 & 6.15 & 6.06 & 6.76 & 6.53 & 6.42 & 6.16 & 6.23 \\
\hline $\mathrm{Zr} / \mathrm{Y}$ & 7.67 & 11.36 & 8.93 & 8.13 & 9.87 & 8.71 & 8.83 & 8.29 & 9.25 & 8.56 & 8.67 & 8.59 \\
\hline $\mathrm{Nb} / \mathrm{Y}$ & 1.46 & 1.93 & 1.18 & 1.27 & 1.48 & 1.42 & 1.46 & 1.23 & 1.42 & 1.33 & 1.41 & 1.38 \\
\hline
\end{tabular}


Table 1 (continued).

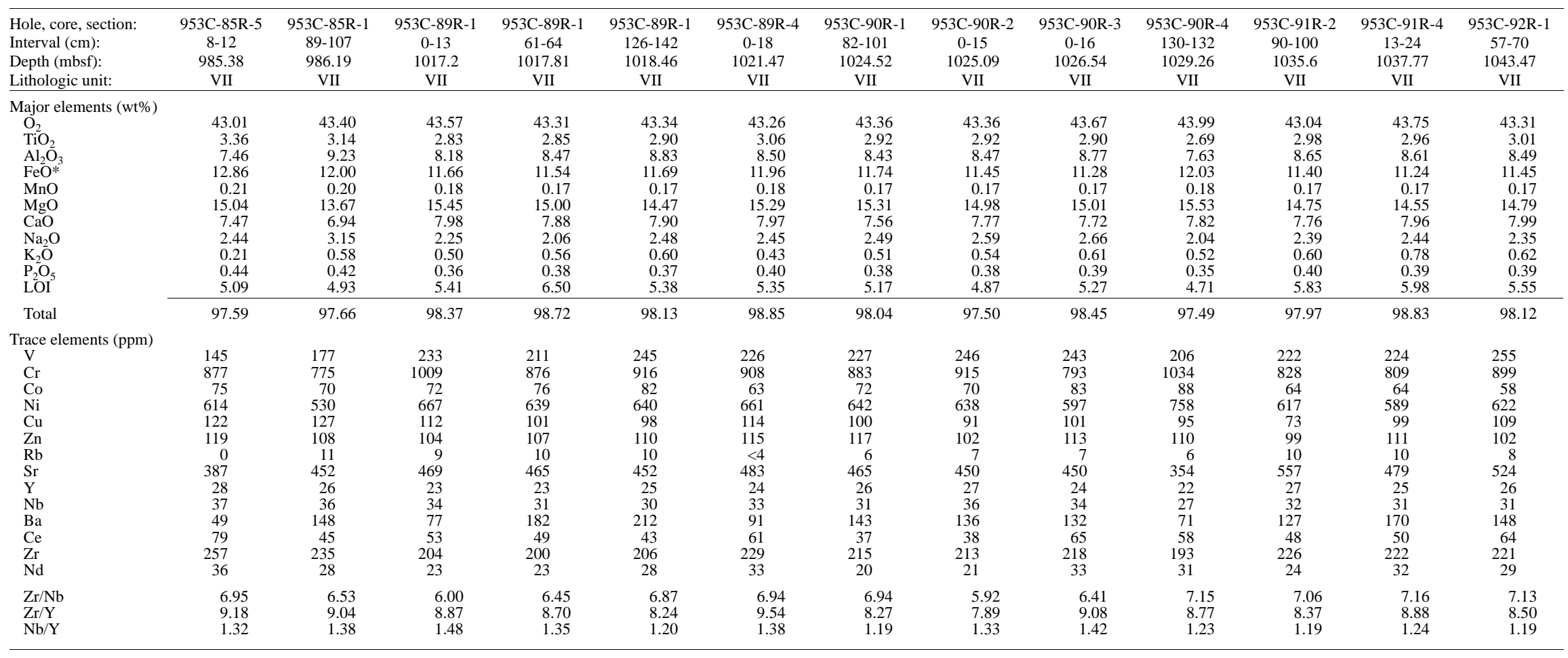


Table 1 (continued).

\begin{tabular}{|c|c|c|c|c|c|c|c|c|c|c|c|c|c|}
\hline $\begin{array}{l}\text { Hole, core, section: } \\
\text { Interval }(\mathrm{cm}): \\
\text { Depth (mbsf): } \\
\text { Lithologic unit: }\end{array}$ & $\begin{array}{c}953-93 R-4 \\
20-24 \\
1057.09 \\
\text { VII }\end{array}$ & $\begin{array}{c}953 \mathrm{C}-94 \mathrm{R}-3 \\
125-146 \\
1066.18 \\
\text { VII } \\
\end{array}$ & $\begin{array}{c}953 \mathrm{C}-95 \mathrm{R}-3 \\
128-150 \\
1075.57 \\
\text { VII } \\
\end{array}$ & $\begin{array}{c}\text { 953C-96R-1 } \\
51-69 \\
1082.01 \\
\text { VII } \\
\end{array}$ & $\begin{array}{c}953 \mathrm{C}-96 \mathrm{R}-4 \\
118-139 \\
1087.03 \\
\text { VII } \\
\end{array}$ & $\begin{array}{c}953 \mathrm{C}-96 \mathrm{R}-6 \\
20-32 \\
1088.74 \\
\text { VII } \\
\end{array}$ & $\begin{array}{c}953 \mathrm{C}-97 \mathrm{R}-2 \\
0-16 \\
1092.47 \\
\text { VII } \\
\end{array}$ & $\begin{array}{c}953 \mathrm{C}-97 \mathrm{R}-4 \\
50-53 \\
1095.8 \\
\text { VII }\end{array}$ & $\begin{array}{c}\text { 953C-98R-1 } \\
0-12 \\
1100.9 \\
\text { VII } \\
\end{array}$ & $\begin{array}{c}\text { 953C-98R-2 } \\
62-64 \\
1102.86 \\
\text { VII } \\
\end{array}$ & $\begin{array}{c}\text { 953C-98R-6 } \\
140-147 \\
1109.13 \\
\text { VII } \\
\end{array}$ & $\begin{array}{c}953 \mathrm{C}-100 \mathrm{R}-4 \\
0-15 \\
1124.39 \\
\text { VII }\end{array}$ & $\begin{array}{c}953 \mathrm{C}-100 \mathrm{R}-4 \\
145-148 \\
1125.84 \\
\text { VII } \\
\end{array}$ \\
\hline $\begin{array}{l}\text { Major elements (wt\%) } \\
\mathrm{SiO}_{2} \\
\mathrm{TiO}_{2} \\
\mathrm{Al}_{2} \mathrm{O}_{3} \\
\mathrm{FeO} \\
\mathrm{MnO} \\
\mathrm{MgO} \\
\mathrm{CaO} \\
\mathrm{Na}_{2} \mathrm{O} \\
\mathrm{K}_{2} \mathrm{O} \\
\mathrm{P}_{2} \mathrm{O}_{5} \\
\mathrm{LOI}\end{array}$ & $\begin{array}{r}45.54 \\
3.30 \\
9.38 \\
12.05 \\
0.18 \\
14.08 \\
8.96 \\
1.97 \\
0.63 \\
0.44 \\
1.54 \\
\end{array}$ & $\begin{array}{r}42.92 \\
3.15 \\
8.61 \\
11.20 \\
0.16 \\
13.76 \\
8.09 \\
2.28 \\
0.87 \\
0.43 \\
5.84 \\
\end{array}$ & $\begin{array}{r}43.17 \\
3.10 \\
9.00 \\
11.36 \\
0.17 \\
13.53 \\
8.24 \\
2.75 \\
0.81 \\
0.44 \\
5.36 \\
\end{array}$ & $\begin{array}{r}42.96 \\
3.36 \\
9.34 \\
11.55 \\
0.17 \\
12.65 \\
8.04 \\
2.96 \\
0.86 \\
0.49 \\
5.46 \\
\end{array}$ & $\begin{array}{r}43.21 \\
3.29 \\
9.46 \\
10.93 \\
0.17 \\
11.29 \\
8.34 \\
2.95 \\
1.25 \\
0.47 \\
6.59 \\
\end{array}$ & $\begin{array}{r}43.05 \\
3.24 \\
9.18 \\
11.06 \\
0.17 \\
12.50 \\
8.32 \\
2.50 \\
1.16 \\
0.46 \\
5.70 \\
\end{array}$ & $\begin{array}{r}42.79 \\
3.33 \\
8.61 \\
11.51 \\
0.17 \\
13.86 \\
8.09 \\
2.47 \\
0.74 \\
0.46 \\
5.80 \\
\end{array}$ & $\begin{array}{r}42.78 \\
3.31 \\
8.84 \\
11.43 \\
0.17 \\
13.19 \\
8.10 \\
2.39 \\
0.81 \\
0.48 \\
6.99 \\
\end{array}$ & $\begin{array}{r}42.70 \\
3.44 \\
8.97 \\
11.61 \\
0.17 \\
13.23 \\
8.17 \\
2.46 \\
0.86 \\
0.47 \\
6.08 \\
\end{array}$ & $\begin{array}{r}42.85 \\
3.25 \\
8.65 \\
11.31 \\
0.17 \\
13.75 \\
8.05 \\
2.13 \\
0.82 \\
0.45 \\
6.86 \\
\end{array}$ & $\begin{array}{r}43.79 \\
3.13 \\
9.24 \\
12.17 \\
0.19 \\
13.79 \\
9.21 \\
1.81 \\
0.63 \\
0.42 \\
4.17 \\
\end{array}$ & $\begin{array}{r}43.28 \\
3.40 \\
9.02 \\
11.18 \\
0.17 \\
13.15 \\
8.56 \\
2.47 \\
0.91 \\
0.47 \\
5.19 \\
\end{array}$ & $\begin{array}{r}41.82 \\
4.11 \\
11.78 \\
10.41 \\
0.19 \\
9.27 \\
8.94 \\
3.85 \\
0.93 \\
0.73 \\
6.23 \\
\end{array}$ \\
\hline Total & 98.07 & 97.31 & 97.93 & 97.84 & 97.95 & 97.34 & 97.83 & 98.49 & 98.16 & 98.29 & 98.55 & 97.80 & 98.26 \\
\hline $\begin{array}{l}\text { Trace elements (ppm) } \\
\text { V } \\
\mathrm{Cr} \\
\mathrm{Co} \\
\mathrm{Ni} \\
\mathrm{Cu} \\
\mathrm{Zn} \\
\mathrm{Rb} \\
\mathrm{Sr} \\
\mathrm{Y} \\
\mathrm{Nb} \\
\mathrm{Ba} \\
\mathrm{Ce} \\
\mathrm{Zr} \\
\mathrm{Nd}\end{array}$ & $\begin{array}{r}216 \\
863 \\
113 \\
606 \\
95 \\
114 \\
0 \\
538 \\
27 \\
30 \\
227 \\
63 \\
237 \\
33\end{array}$ & $\begin{array}{r}236 \\
838 \\
63 \\
548 \\
127 \\
102 \\
14 \\
485 \\
27 \\
36 \\
173 \\
62 \\
241 \\
27\end{array}$ & $\begin{array}{r}248 \\
782 \\
51 \\
527 \\
101 \\
99 \\
11 \\
464 \\
28 \\
34 \\
76 \\
50 \\
238 \\
28\end{array}$ & $\begin{array}{r}232 \\
707 \\
66 \\
513 \\
106 \\
101 \\
9 \\
457 \\
30 \\
45 \\
167 \\
64 \\
267 \\
30\end{array}$ & $\begin{array}{r}263 \\
693 \\
46 \\
390 \\
86 \\
99 \\
20 \\
556 \\
27 \\
39 \\
227 \\
54 \\
250 \\
24\end{array}$ & $\begin{array}{r}252 \\
814 \\
44 \\
491 \\
114 \\
101 \\
17 \\
486 \\
30 \\
43 \\
244 \\
68 \\
262 \\
32\end{array}$ & $\begin{array}{r}231 \\
780 \\
59 \\
552 \\
98 \\
100 \\
9 \\
505 \\
26 \\
38 \\
104 \\
63 \\
267 \\
36\end{array}$ & $\begin{array}{r}204 \\
764 \\
61 \\
530 \\
107 \\
110 \\
13 \\
524 \\
24 \\
32 \\
165 \\
56 \\
253 \\
34\end{array}$ & $\begin{array}{r}242 \\
767 \\
45 \\
546 \\
120 \\
107 \\
11 \\
513 \\
30 \\
40 \\
191 \\
54 \\
270 \\
42\end{array}$ & $\begin{array}{r}216 \\
780 \\
52 \\
541 \\
101 \\
103 \\
14 \\
522 \\
24 \\
33 \\
185 \\
76 \\
250 \\
36\end{array}$ & $\begin{array}{r}242 \\
899 \\
89 \\
634 \\
80 \\
109 \\
0 \\
940 \\
26 \\
35 \\
149 \\
73 \\
191 \\
30\end{array}$ & $\begin{array}{r}245 \\
792 \\
40 \\
492 \\
105 \\
101 \\
12 \\
477 \\
26 \\
35 \\
171 \\
49 \\
268 \\
30\end{array}$ & $\begin{array}{r}250 \\
320 \\
34 \\
177 \\
92 \\
105 \\
15 \\
472 \\
31 \\
56 \\
245 \\
91 \\
377 \\
49\end{array}$ \\
\hline $\begin{array}{l}\mathrm{Zr} / \mathrm{Nb} \\
\mathrm{Zr} / \mathrm{Y} \\
\mathrm{Nb} / \mathrm{Y}\end{array}$ & $\begin{array}{l}7.90 \\
8.78 \\
1.11\end{array}$ & $\begin{array}{l}6.69 \\
8.93 \\
1.33\end{array}$ & $\begin{array}{l}7.00 \\
8.50 \\
1.21\end{array}$ & $\begin{array}{l}5.93 \\
8.90 \\
1.50\end{array}$ & $\begin{array}{l}6.41 \\
9.26 \\
1.44\end{array}$ & $\begin{array}{l}6.09 \\
8.73 \\
1.43\end{array}$ & $\begin{array}{r}7.03 \\
10.27 \\
1.46\end{array}$ & $\begin{array}{r}7.91 \\
10.54 \\
1.33\end{array}$ & $\begin{array}{l}6.75 \\
9.00 \\
1.33\end{array}$ & $\begin{array}{r}7.58 \\
10.42 \\
1.38\end{array}$ & $\begin{array}{l}5.46 \\
7.35 \\
1.35\end{array}$ & $\begin{array}{r}7.66 \\
10.31 \\
1.35\end{array}$ & $\begin{array}{r}6.73 \\
12.16 \\
1.81\end{array}$ \\
\hline
\end{tabular}


Table 1 (continued)

\begin{tabular}{|c|c|c|c|c|c|c|c|c|c|}
\hline $\begin{array}{l}\text { Hole, core, section: } \\
\text { Interval }(\mathrm{cm}): \\
\text { Depth }(\mathrm{mbsf}): \\
\text { Lithologic unit: }\end{array}$ & $\begin{array}{c}953 \mathrm{C}-101 \mathrm{R}-4 \\
1-13 \\
1134.03 \\
\text { VII }\end{array}$ & $\begin{array}{c}\text { 953C-101R-5 } \\
78-80 \\
1136.3 \\
\text { VII } \\
\end{array}$ & $\begin{array}{c}953 \mathrm{C}-102 \mathrm{R}-1 \\
8-28 \\
1139.48 \\
\text { VII } \\
\end{array}$ & $\begin{array}{c}953 \mathrm{C}-102 \mathrm{R}-2 \\
80-90 \\
1141.35 \\
\text { VII }\end{array}$ & $\begin{array}{c}\text { 953C-103R-2 } \\
42-52 \\
1150.77 \\
\text { VII } \\
\end{array}$ & $\begin{array}{c}953 \mathrm{C}-103 \mathrm{R}-4 \\
114-127 \\
1154.36 \\
\text { VII } \\
\end{array}$ & $\begin{array}{c}953 \mathrm{C}-103 \mathrm{R}-6 \\
67-70 \\
1156.85 \\
\text { VII } \\
\end{array}$ & $\begin{array}{c}953 \mathrm{C}-103 \mathrm{R}-6 \\
116-126 \\
1157.34 \\
\text { VII } \\
\end{array}$ & $\begin{array}{c}953 \mathrm{C}-101 \mathrm{R}-3 \\
8-22 \\
1132.63 \\
\text { VII } \\
\end{array}$ \\
\hline $\begin{array}{l}\text { Major elements (wt\%) } \\
\mathrm{SiO}_{2} \\
\mathrm{TiO}_{2} \\
\mathrm{Al}_{2} \mathrm{O}_{3} \\
\mathrm{FeO} \\
\mathrm{MnO} \\
\mathrm{MgO} \\
\mathrm{CaO} \\
\mathrm{Na}_{2} \mathrm{O} \\
\mathrm{K}_{2} \mathrm{O} \\
\mathrm{P}_{2} \mathrm{O}_{5} \\
\mathrm{LOI}\end{array}$ & $\begin{array}{r}43.53 \\
3.27 \\
8.82 \\
10.81 \\
0.16 \\
13.56 \\
7.77 \\
2.32 \\
1.08 \\
0.47 \\
6.59 \\
\end{array}$ & $\begin{array}{r}43.73 \\
4.04 \\
8.89 \\
12.83 \\
0.19 \\
11.42 \\
9.51 \\
2.18 \\
0.99 \\
0.48 \\
3.82 \\
\end{array}$ & $\begin{array}{r}42.73 \\
3.62 \\
8.62 \\
11.29 \\
0.17 \\
12.93 \\
8.85 \\
2.27 \\
0.97 \\
0.53 \\
6.74 \\
\end{array}$ & $\begin{array}{r}42.73 \\
3.91 \\
9.70 \\
10.94 \\
0.17 \\
11.41 \\
8.59 \\
2.60 \\
1.11 \\
0.56 \\
6.49 \\
\end{array}$ & $\begin{array}{r}42.83 \\
3.71 \\
8.81 \\
11.97 \\
0.18 \\
12.83 \\
8.16 \\
2.29 \\
0.81 \\
0.54 \\
5.63 \\
\end{array}$ & $\begin{array}{r}42.71 \\
3.62 \\
8.88 \\
11.33 \\
0.17 \\
12.35 \\
8.46 \\
2.35 \\
1.02 \\
0.52 \\
6.14 \\
\end{array}$ & $\begin{array}{r}42.57 \\
3.56 \\
9.16 \\
11.34 \\
0.17 \\
12.03 \\
8.38 \\
2.50 \\
0.87 \\
0.54 \\
6.85 \\
\end{array}$ & $\begin{array}{r}42.73 \\
3.59 \\
8.88 \\
11.38 \\
0.17 \\
12.04 \\
8.18 \\
2.78 \\
0.75 \\
0.54 \\
6.00 \\
\end{array}$ & $\begin{array}{r}42.95 \\
3.30 \\
9.01 \\
11.22 \\
0.15 \\
13.06 \\
7.93 \\
2.32 \\
1.01 \\
0.46 \\
6.85 \\
\end{array}$ \\
\hline Total & 98.38 & 98.08 & 98.72 & 98.21 & 97.76 & 97.55 & 97.97 & 97.04 & 98.26 \\
\hline $\begin{array}{l}\text { Trace elements (ppm) } \\
\mathrm{V} \\
\mathrm{Cr} \\
\mathrm{Co} \\
\mathrm{Ni} \\
\mathrm{Cu} \\
\mathrm{Zn} \\
\mathrm{Rb} \\
\mathrm{Sr} \\
\mathrm{Y} \\
\mathrm{Nb} \\
\mathrm{Ba} \\
\mathrm{Ce} \\
\mathrm{Zr} \\
\mathrm{Nd}\end{array}$ & $\begin{array}{r}235 \\
742 \\
70 \\
498 \\
119 \\
104 \\
16 \\
490 \\
27 \\
37 \\
211 \\
85 \\
256 \\
41\end{array}$ & $\begin{array}{r}271 \\
941 \\
74 \\
479 \\
64 \\
120 \\
16 \\
653 \\
25 \\
40 \\
196 \\
82 \\
254 \\
36\end{array}$ & $\begin{array}{r}249 \\
752 \\
53 \\
456 \\
75 \\
101 \\
12 \\
516 \\
30 \\
41 \\
168 \\
77 \\
316 \\
48\end{array}$ & $\begin{array}{r}267 \\
654 \\
52 \\
370 \\
121 \\
118 \\
15 \\
582 \\
31 \\
40 \\
242 \\
76 \\
347 \\
41\end{array}$ & $\begin{array}{r}247 \\
771 \\
67 \\
533 \\
91 \\
108 \\
10 \\
534 \\
29 \\
41 \\
154 \\
78 \\
314 \\
38\end{array}$ & $\begin{array}{r}247 \\
763 \\
90 \\
501 \\
115 \\
105 \\
14 \\
544 \\
29 \\
37 \\
133 \\
76 \\
310 \\
33\end{array}$ & $\begin{array}{r}202 \\
636 \\
68 \\
459 \\
108 \\
112 \\
12 \\
568 \\
25 \\
40 \\
120 \\
88 \\
302 \\
38\end{array}$ & $\begin{array}{r}241 \\
698 \\
70 \\
482 \\
110 \\
103 \\
7 \\
604 \\
31 \\
48 \\
109 \\
66 \\
312 \\
32\end{array}$ & $\begin{array}{r}220 \\
808 \\
53 \\
549 \\
108 \\
105 \\
15 \\
515 \\
28 \\
39 \\
198 \\
55 \\
258 \\
44\end{array}$ \\
\hline $\begin{array}{l}\mathrm{Zr} / \mathrm{Nb} \\
\mathrm{Zr} / \mathrm{Y} \\
\mathrm{Nb} / \mathrm{Y}\end{array}$ & $\begin{array}{l}6.92 \\
9.48 \\
1.37\end{array}$ & $\begin{array}{r}6.35 \\
10.16 \\
1.60\end{array}$ & $\begin{array}{r}7.71 \\
10.53 \\
1.37\end{array}$ & $\begin{array}{r}8.68 \\
11.19 \\
1.29\end{array}$ & $\begin{array}{r}7.66 \\
10.83 \\
1.41\end{array}$ & $\begin{array}{r}8.38 \\
10.69 \\
1.28\end{array}$ & $\begin{array}{r}7.55 \\
12.08 \\
1.60\end{array}$ & $\begin{array}{r}6.50 \\
10.06 \\
1.55\end{array}$ & $\begin{array}{l}6.62 \\
9.21 \\
1.39\end{array}$ \\
\hline
\end{tabular}


Table 2. XRF major and trace element analyses of basalt clasts, Hole 953C.

\begin{tabular}{|c|c|c|c|c|c|}
\hline $\begin{array}{l}\text { Hole, core, section: } \\
\text { Interval (cm): } \\
\text { Depth (mbsf): } \\
\text { Lithologic unit: }\end{array}$ & $\begin{array}{c}953 \mathrm{C}-90 \mathrm{R}-5 \\
0-8 \\
1029.54 \\
\text { VII } \\
\end{array}$ & $\begin{array}{c}953 \mathrm{C}-90 \mathrm{R}-5 \\
49-55 \\
1030.03 \\
\text { VII } \\
\end{array}$ & $\begin{array}{c}953 \mathrm{C}-93 \mathrm{R}-2 \\
8-24 \\
1053.78 \\
\text { VII } \\
\end{array}$ & $\begin{array}{c}\text { 953C-98R-6 } \\
34-45 \\
1108.74 \\
\text { VII } \\
\end{array}$ & $\begin{array}{c}953 \mathrm{C}-101 \mathrm{R}-2 \\
86-106 \\
1131.91 \\
\text { VII }\end{array}$ \\
\hline $\begin{array}{l}\text { Major elements } \\
\text { (wt\%) } \\
\mathrm{SiO}_{2} \\
\mathrm{TiO}_{2} \\
\mathrm{Al}_{2} \mathrm{O}_{3} \\
\mathrm{FeO}^{*} \\
\mathrm{MnO} \\
\mathrm{MgO} \\
\mathrm{CaO} \\
\mathrm{Na}{ }_{2} \mathrm{O} \\
\mathrm{K}_{2} \mathrm{O} \\
\mathrm{P}_{2} \mathrm{O}_{5} \\
\mathrm{LOI}\end{array}$ & $\begin{array}{r}45.11 \\
3.79 \\
9.60 \\
12.37 \\
0.19 \\
13.02 \\
9.76 \\
1.93 \\
1.06 \\
0.56 \\
1.06 \\
\end{array}$ & $\begin{array}{r}44.58 \\
3.45 \\
9.72 \\
12.16 \\
0.18 \\
13.82 \\
9.61 \\
1.87 \\
0.77 \\
0.48 \\
1.97 \\
\end{array}$ & $\begin{array}{r}44.38 \\
3.43 \\
10.23 \\
10.77 \\
0.17 \\
8.72 \\
9.91 \\
3.43 \\
1.79 \\
0.46 \\
4.76 \\
\end{array}$ & $\begin{array}{r}41.81 \\
3.57 \\
9.48 \\
12.54 \\
0.19 \\
13.08 \\
10.25 \\
1.36 \\
0.49 \\
0.46 \\
4.57 \\
\end{array}$ & $\begin{array}{r}45.17 \\
3.75 \\
9.54 \\
11.89 \\
0.18 \\
14.19 \\
9.40 \\
1.72 \\
0.82 \\
0.49 \\
1.54 \\
\end{array}$ \\
\hline Total & 98.45 & 98.61 & 98.05 & 97.80 & 98.69 \\
\hline $\begin{array}{l}\text { Trace elements } \\
\text { (ppm) } \\
\mathrm{V} \\
\mathrm{Cr} \\
\mathrm{Co} \\
\mathrm{Ni} \\
\mathrm{Cu} \\
\mathrm{Zn} \\
\mathrm{Rb} \\
\mathrm{Sr} \\
\mathrm{Y} \\
\mathrm{Nb} \\
\mathrm{Ba} \\
\mathrm{La} \\
\mathrm{Ce} \\
\mathrm{Zr} \\
\mathrm{Pr} \\
\mathrm{Nd}\end{array}$ & $\begin{array}{r}298 \\
875 \\
63 \\
512 \\
137 \\
111 \\
14 \\
538 \\
29 \\
50 \\
259 \\
125 \\
89 \\
291 \\
20 \\
51\end{array}$ & $\begin{array}{r}244 \\
830 \\
55 \\
544 \\
93 \\
110 \\
0 \\
799 \\
28 \\
38 \\
279 \\
123 \\
76 \\
238 \\
18 \\
38\end{array}$ & $\begin{array}{r}228 \\
393 \\
57 \\
186 \\
142 \\
105 \\
11 \\
412 \\
29 \\
44 \\
665 \\
117 \\
71 \\
252 \\
13 \\
31\end{array}$ & $\begin{array}{r}226 \\
947 \\
75 \\
697 \\
127 \\
120 \\
0 \\
673 \\
32 \\
39 \\
133 \\
93 \\
59 \\
266 \\
15 \\
34\end{array}$ & $\begin{array}{r}214 \\
849 \\
57 \\
563 \\
87 \\
116 \\
0 \\
668 \\
33 \\
38 \\
296 \\
90 \\
50 \\
285 \\
15 \\
39\end{array}$ \\
\hline $\begin{array}{l}\mathrm{Zr} / \mathrm{Nb} \\
\mathrm{Zr} / \mathrm{Y} \\
\mathrm{Nb} / \mathrm{Y}\end{array}$ & $\begin{array}{r}5.82 \\
10.03 \\
1.72\end{array}$ & $\begin{array}{l}6.26 \\
8.50 \\
1.36\end{array}$ & $\begin{array}{l}5.73 \\
8.69 \\
1.52\end{array}$ & $\begin{array}{l}6.82 \\
8.31 \\
1.22\end{array}$ & $\begin{array}{l}7.50 \\
8.64 \\
1.15\end{array}$ \\
\hline
\end{tabular}

Table 3. XRF major and trace element analyses of whole rock samples, Hole 954B.

\begin{tabular}{|c|c|c|c|}
\hline $\begin{array}{l}\text { Hole, core, section: } \\
\text { Interval (cm): } \\
\text { Depth (mbsf): } \\
\text { Lithologic unit }\end{array}$ & $\begin{array}{c}\text { 954B-36R-1 } \\
7-12 \\
417.37 \\
\text { IV } \\
\end{array}$ & $\begin{array}{c}\text { 954B-37R-1 } \\
137-140 \\
428.17 \\
\text { IV } \\
\end{array}$ & $\begin{array}{c}\text { 954B-38R-1 } \\
15-16 \\
436.45 \\
\text { IV }\end{array}$ \\
\hline \multicolumn{4}{|l|}{$\begin{array}{l}\text { Major elements } \\
\text { (wt } \%)\end{array}$} \\
\hline $\begin{array}{l}\mathrm{SiO}_{2} \\
\mathrm{TiO}_{2} \\
\mathrm{Al}_{2} \mathrm{O}_{3} \\
\mathrm{FeO} \\
\mathrm{MnO} \\
\mathrm{MgO} \\
\mathrm{CaO} \\
\mathrm{Na}{ }_{2} \mathrm{O} \\
\mathrm{K}_{2} \mathrm{O} \\
\mathrm{P}_{2} \mathrm{O}_{5} \\
\mathrm{SO}_{3}\end{array}$ & $\begin{array}{r}45.24 \\
4.75 \\
11.73 \\
10.19 \\
0.17 \\
6.53 \\
12.05 \\
2.36 \\
1.49 \\
0.69\end{array}$ & $\begin{array}{r}45.75 \\
4.40 \\
11.22 \\
10.92 \\
0.24 \\
8.38 \\
10.01 \\
2.33 \\
0.92 \\
0.45\end{array}$ & $\begin{array}{r}42.84 \\
5.49 \\
10.61 \\
12.92 \\
0.17 \\
8.55 \\
10.29 \\
2.14 \\
1.34 \\
0.76\end{array}$ \\
\hline LOI & 3.05 & 3.02 & \\
\hline Total & 98.26 & 97.65 & 97.10 \\
\hline \multicolumn{4}{|l|}{$\begin{array}{l}\text { Trace elements } \\
(\mathrm{ppm})\end{array}$} \\
\hline $\mathrm{Cr}$ & 218 & 605 & 325 \\
\hline $\mathrm{Ni}$ & 126 & 216 & 189 \\
\hline $\mathrm{Cu}$ & 219 & 60 & 94 \\
\hline $\mathrm{Zn}$ & 140 & 128 & 127 \\
\hline $\mathrm{Rb}$ & 29 & 14 & 15 \\
\hline $\mathrm{Sr}$ & 768 & 533 & 619 \\
\hline $\mathrm{Y}$ & 33 & 32 & 36 \\
\hline $\mathrm{Nb}$ & 72 & 46 & 56 \\
\hline $\mathrm{Ba}$ & 356 & 171 & 238 \\
\hline $\mathrm{Ce}$ & 110 & 101 & 105 \\
\hline $\mathrm{Zr}$ & 362 & 378 & 442 \\
\hline $\mathrm{Zr} / \mathrm{Nb}$ & 5.03 & 8.22 & 7.89 \\
\hline $\mathrm{Zr} / \mathrm{Y}$ & 1097 & 1181 & 1228 \\
\hline $\mathrm{Nb} / \mathrm{Y}$ & 2.18 & 1.44 & 1.56 \\
\hline
\end{tabular}

ures 19A, B, and 20 (top two illustrations). Sixty-four polished and 18 covered thin sections were studied from almost all of the chemically analyzed samples. Although most of the analyzed bulk rock samples are impure, inasmuch as they represent mixtures of different volcanic and nonvolcanic components, the major and trace element concentrations mirror the chemical characteristics of the shield basalt lavas on land quite well. Element ratios of relatively immobile trace elements are almost identical to those of the subaerial shield lavas. $\mathrm{The} \mathrm{Zr} / \mathrm{Nb}$ ratio of the analyzed whole rocks and basalt clasts ranges from 6.9 (Site 956) to 7.2 (Site 953) compared to 7.1 for the subaerial basalts. The $\mathrm{Zr} / \mathrm{Y}$ ratio ranges from 9.6 (Site 953) to 10.5 (Site 956) compared to 10.5 for the subaerial basalts. The remarkable constancy of these ratios argues for a fairly homogeneous magma source during the late submarine and subaerial shield stage.

All rocks show massive $\mathrm{Ca}-\mathrm{loss} . \mathrm{Mg}$ and alkalis are invariably added to the rocks, apart from $\mathrm{H}_{2} \mathrm{O}$. Na addition is less pronounced in one group of rocks in Site 956, whereas K-addition is more pronounced in one group of analyses in Site 956 . Fe is enriched slightly in rocks from both sites. As expected, clasts are generally less strongly altered than hyaloclastites.

Basaltic tuffs, lapillistones, and breccias in Units V, VI, and VII of Hole 953C and Unit $\mathrm{V}$ of Hole 956B have low $\mathrm{SiO}_{2}$ and very high $\mathrm{MgO}$ concentrations (up to $17 \mathrm{wt} \%$; Figs. 19A, 20 [top illustration]). They also have high total $\mathrm{Fe}$ and $\mathrm{TiO}_{2}$, a characteristic of mafic magmas from the Canary Islands (Schmincke, 1982). They have low abundances of incompatible trace elements and high concentrations of Ni (mostly 400-860 ppm), Cr (161-1100 ppm), and V (150-780 ppm; Figs. 19B and 20 [second illustration from top]).

The bulk of the hyaloclastites, especially from Site 953, is more primitive than the bulk of the subaerial basalts as shown by clast and bulk rock major and trace element composition indicating an overall more primitive stage of evolution of the submarine sequence. In par- 
Table 4. XRF major and trace element analyses of basalt clasts, Hole 954B.

\begin{tabular}{|c|c|c|c|}
\hline $\begin{array}{l}\text { Hole, core, section: } \\
\text { Interval }(\mathrm{cm}): \\
\text { Depth }(\mathrm{mbsf}): \\
\text { Lithologic unit }\end{array}$ & $\begin{array}{c}\text { 954B-35R-1 } \\
72-78 \\
408.72 \\
\text { IV } \\
\end{array}$ & $\begin{array}{c}954 \mathrm{~B}-38 \mathrm{R}-3 \\
64-67 \\
439.64 \\
\text { IV } \\
\end{array}$ & $\begin{array}{c}\text { 954B-39R-3 } \\
38-48 \\
445.38 \\
\text { IV } \\
\end{array}$ \\
\hline $\begin{array}{l}\text { Major elements } \\
\text { (wt.\%) }\end{array}$ & & & \\
\hline $\begin{array}{l}\mathrm{SiO}_{2} \\
\mathrm{TiO}_{2} \\
\mathrm{Al}_{2} \mathrm{O}_{3} \\
\mathrm{FeO} \\
\mathrm{MnO} \\
\mathrm{MgO} \\
\mathrm{CaO} \\
\mathrm{Na}_{2} \mathrm{O} \\
\mathrm{K}_{2} \mathrm{O} \\
\mathrm{P}_{2} \mathrm{O}_{5} \\
\mathrm{LOI}\end{array}$ & $\begin{array}{r}48.44 \\
4.50 \\
13.86 \\
11.29 \\
0.15 \\
4.61 \\
9.61 \\
2.86 \\
1.22 \\
0.57 \\
1.59\end{array}$ & $\begin{array}{r}44.17 \\
5.10 \\
11.92 \\
12.24 \\
0.18 \\
7.14 \\
9.79 \\
2.05 \\
1.33 \\
0.71 \\
4.15 \\
\end{array}$ & $\begin{array}{r}42.49 \\
5.37 \\
11.53 \\
9.09 \\
0.15 \\
6.38 \\
12.49 \\
3.00 \\
1.70 \\
0.89 \\
4.42 \\
\end{array}$ \\
\hline Total & 98.70 & 98.78 & 97.51 \\
\hline $\begin{array}{l}\text { Trace elements } \\
\text { (ppm) } \\
\mathrm{V} \\
\mathrm{Cr} \\
\mathrm{Co} \\
\mathrm{Ni} \\
\mathrm{Cu} \\
\mathrm{Zn} \\
\mathrm{Rb} \\
\mathrm{Sr} \\
\mathrm{Y} \\
\mathrm{Nb} \\
\mathrm{Ba} \\
\mathrm{La} \\
\mathrm{Ce} \\
\mathrm{Zr} \\
\mathrm{Pr} \\
\mathrm{Nd}\end{array}$ & $\begin{array}{r}331 \\
71 \\
61 \\
83 \\
117 \\
120 \\
32 \\
649 \\
37 \\
56 \\
284 \\
122 \\
100 \\
380 \\
18 \\
56\end{array}$ & $\begin{array}{r}305 \\
101 \\
38 \\
100 \\
103 \\
136 \\
24 \\
640 \\
35 \\
64 \\
228 \\
121 \\
93 \\
445 \\
24 \\
52\end{array}$ & $\begin{array}{r}330 \\
127 \\
32 \\
134 \\
118 \\
161 \\
22 \\
969 \\
44 \\
83 \\
464 \\
130 \\
135 \\
512 \\
24 \\
64\end{array}$ \\
\hline $\begin{array}{l}\mathrm{Zr} / \mathrm{Nb} \\
\mathrm{Zr} / \mathrm{Y} \\
\mathrm{Nb} / \mathrm{Y}\end{array}$ & $\begin{array}{r}6.79 \\
10.27 \\
5.07\end{array}$ & $\begin{array}{r}6.95 \\
12.71 \\
3.56\end{array}$ & $\begin{array}{r}6.17 \\
11.64 \\
5.59\end{array}$ \\
\hline
\end{tabular}

ticular, the very high Ni concentration and the abundance of large olivine pseudomorphs found in some units such as Unit VII of Hole 953C indicate that olivine-rich to picritic magmas were dominant during the submarine and shallow emergent shield phases at this stage. About half of the rocks analyzed from Site 956 are less mafic than those from Site 953C. Plagioclase-rich deposits including lapilli with more evolved fresh glass occur between 560 and $590 \mathrm{~m}$ at Hole 956B southwest of Gran Canaria (Fig. 7; Table 16).

\section{LITHOSTRATIGRAPHY}

\section{Hole 953C}

The almost 300-m-thick clastic deposits at Hole 953C (Cores 157953C-71R through 103R, 850-1159 mbsf) were subdivided by the shipboard party, from the base upward, into 190-m-thick Unit VII (Sections 157-953C-83R-4 through 103R-7, 969-1159 mbsf); $80 \mathrm{~m}$ thick Unit VI (Sections 157-953C-75R-1 through 83R-3, 889-969 mbsf) and $49 \mathrm{~m}$ thick Unit V (Sections 157-953C-71R-1 through 74R-6, 850-889 mbsf; Fig. 2).

\section{Unit VII}

Unit VII consists entirely of dark green hyaloclastite tuffs, lapillistones, and breccias that were emplaced as 16 strongly graded debris flows. The unit lacks interbedded fine-grained biogenic sediments and appears to have been deposited extremely rapidly. Recovery $(75 \%)$ is very good to excellent. It is the most homogeneous of all Leg 157 hyaloclastite units. Altered sideromelane clasts and minor tachylite, crystalline basalt, and crystals of clinopyroxene, al- tered olivine, Fe-Ti oxides, and rare plagioclase are the major components. Subaerially erupted basalt clasts are rare. Most common are hyaloclastite tuff and lapillistone debrites (up to $20 \mathrm{~m}$ thick) that grade downward into very poorly sorted clast-rich breccias. Basalt clasts in a single unit can vary from $<1 \mathrm{~cm}$ in the upper tuffaceous part to $>20 \mathrm{~cm}$ in the basal breccia. Medium bedded hyaloclastite tuff units with minor grading and few large basalt clasts are parallel laminated and some are cross-bedded at the base. In Core 157-95398R, a thick-bedded, hyaloclastite lapillistone overlies a laminated hyaloclastite tuff. Below this contact, the laminations in the upper part of the tuff are deformed and convoluted, suggesting slumping when the overlying lapillistone was deposited. Many hyaloclastite tuffs in this stratigraphic interval except for the top beds are dominated by nonvesicular to slightly vesicular shards.

\section{Unit VI}

Unit VI (80 m thick) consists largely of hyaloclastite tuff, lapillistone, and lithic breccia that commonly forms graded beds. Individual sedimentation units exceed $2 \mathrm{~m}$ in thickness contrasting with the much thicker units of Unit VII. The extremely low recovery rate $(15 \%-20 \%)$ prevents good characterization of the unit, however. Breccia composed of basalt clasts dominates in Core 157-953C-76R and 78R, whereas hyaloclastite tuffs and lapillistones make up beds several meters thick in Cores 157-953C-77R through 81R and 83R. The major components are completely altered vesicular sideromelane shards, tachylite and crystallized basalt, clinopyroxene, plagioclase, and minor biogenic debris. Large fragments of variably altered basalt ranging up to $20 \mathrm{~cm}$ in diameter based on lengths in the core are common. Most are moderately vesicular, many of the vesicles being filled by zeolites. Some are rounded, but most are angular to subangular. A basaltic lapillistone contains a pillow basalt fragment with a welldeveloped quenched rim $\sim 0.5 \mathrm{~cm}$ thick in Core 157-953C-78R. Altered sideromelane shards are moderately to highly vesicular in the upper part of the unit (Pl. 1, Fig. 1), grading downward to nonvesicular to slightly vesicular coincident with an increase in the abundance of picritic basalt lithic clasts. Nannofossil mixed sedimentary rocks, nannofossil claystones, and claystones, making up $<10 \mathrm{vol} \%$ of the recovered material, occur between the thicker and coarser grained lapillistone and breccia in Cores 157-953C-79R and 83R, where they form turbidite sequences grading from lithic-crystal siltstones and sandstones to nannofossil claystones.

\section{Unit V}

In the basaltic crystal-lithic siltstones and sandstones, totaling 49 $\mathrm{m}$ thick, a few coarser grained and thicker sandstones to lapillistones occur, that is, Sections 157-953C-71R-4 and 71R-3 (Figs. 21-23). The volcaniclastic turbidites are $6-40 \mathrm{~cm}$ thick. When extrapolated to the nonrecovered intervals, we estimate $\sim 300$ turbidites of which $\sim 90 \%$ in the recovered cores are dominantly volcanic in composition. Most turbidites have sharp bases and bioturbated tops and grade from basal lithic-crystal siltstone and sandstone into bioturbated nannofossil claystone. Many show changes from structureless tops through parallel-laminated in the middle to cross-laminated at the base. The main components are angular to subrounded tachylite, lesser amounts of altered sideromelane fragments, dominantly blocky, and variable but smaller amounts of crystalline basaltic clasts.

The turbidites contain up to $\sim 20 \%$ of biogenic debris, chiefly foraminifers, and are interlayered with nannofossil ooze turbidites. Thin beds of clayey nannofossil mixed pelagic sediments $(<10 \%$ of the unit) occur between the graded turbidites. The relative proportion of volcanic vs. nonvolcanic material is estimated at 90:10. Assuming a nonvolcanic sedimentation rate equal to that of the mostly nonvolcanic sediments of Unit III $(20 \mathrm{~m} / \mathrm{m}$.y. $)$, this $50 \mathrm{~m}$ interval was deposited probably in $<0.5$ m.y. 
Table 5. XRF major and trace element analyses of whole rock samples, Hole 956B

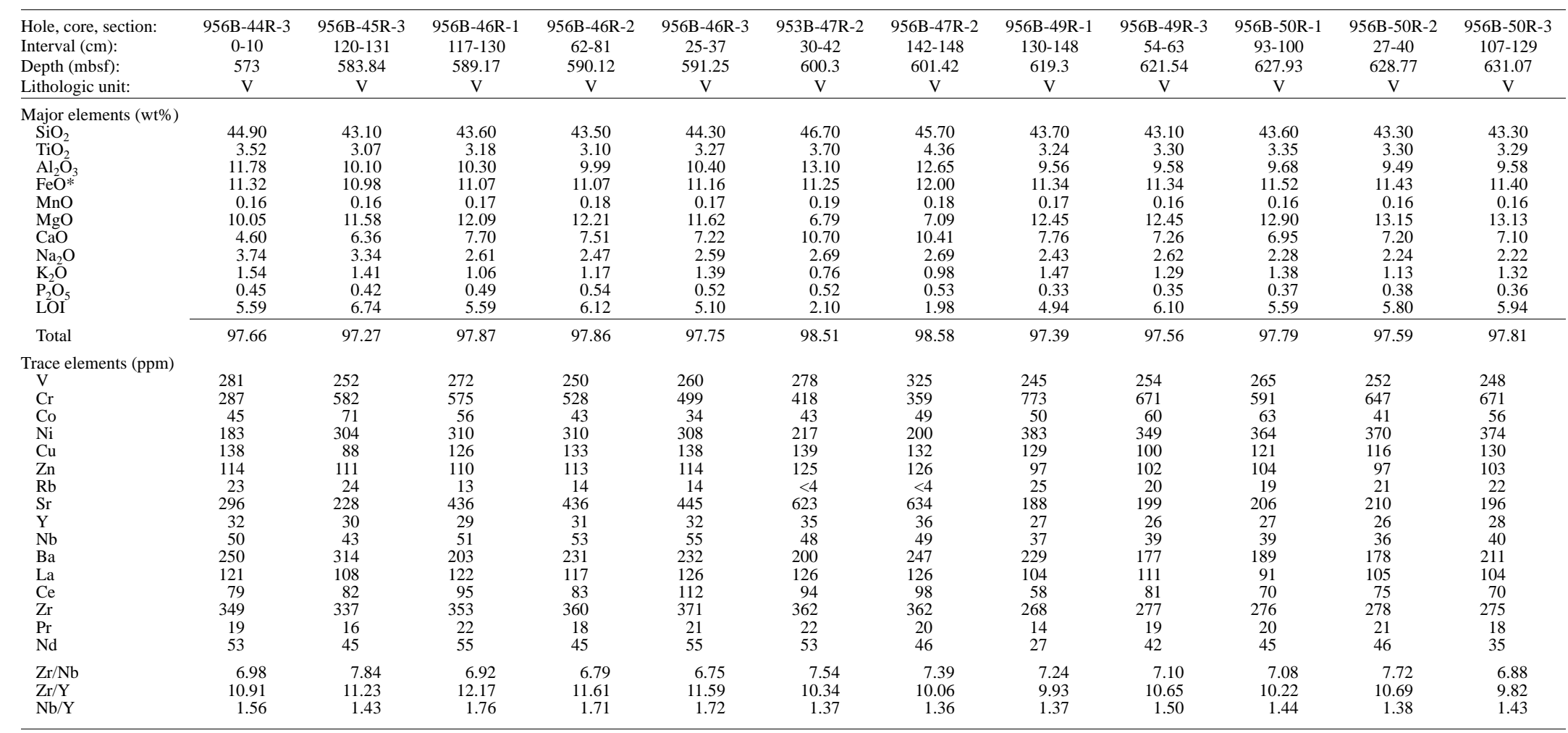


Table 5 (continued).

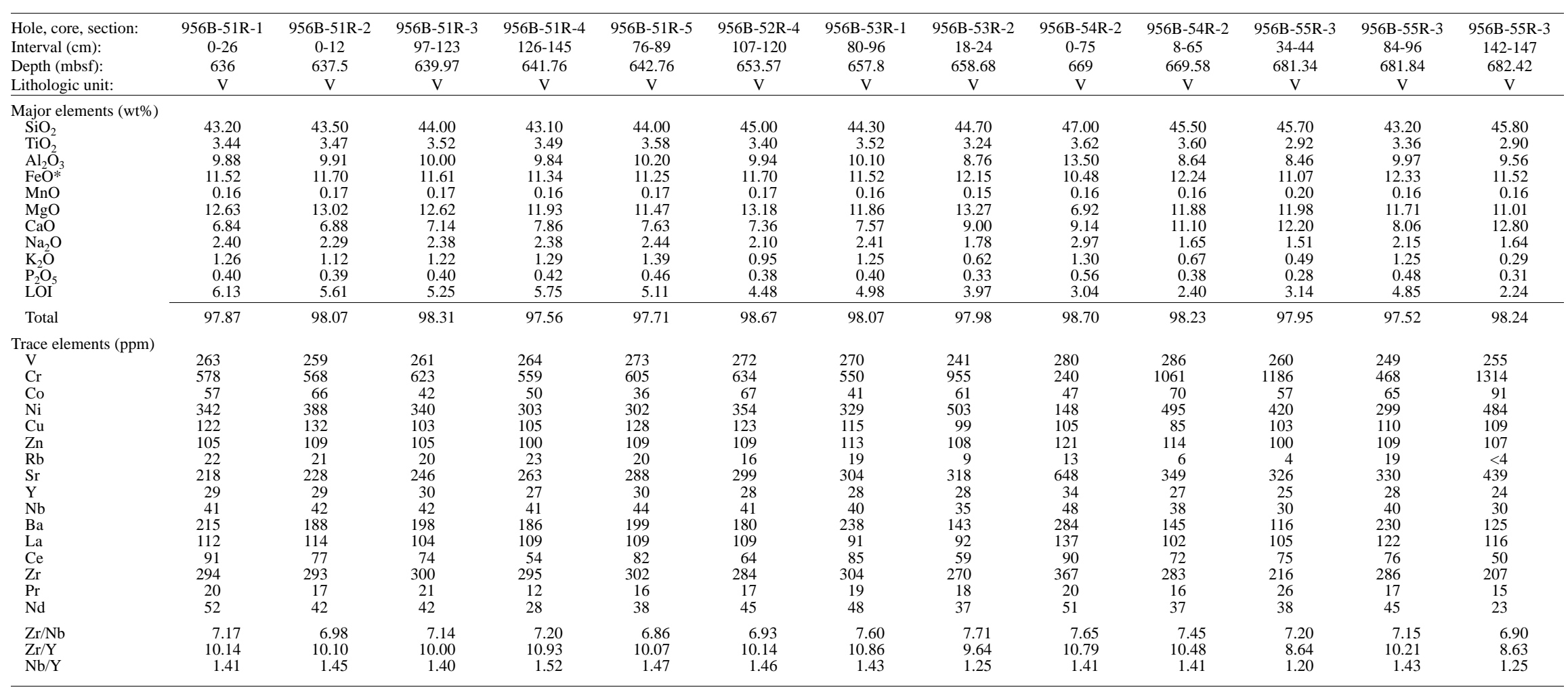


Table 5 (continued).

\begin{tabular}{|c|c|c|c|c|c|c|}
\hline $\begin{array}{l}\text { Hole, core, section: } \\
\text { Interval }(\mathrm{cm}) \text { : } \\
\text { Depth (mbsf): } \\
\text { Lithologic unit }\end{array}$ & $\begin{array}{c}\text { 956B-56R-2 } \\
89-103 \\
690.89 \\
\text { V }\end{array}$ & $\begin{array}{c}956 \mathrm{~B}-56 \mathrm{R}-3 \\
69-77 \\
692.19 \\
\mathrm{~V}\end{array}$ & $\begin{array}{c}956 \mathrm{~B}-57 \mathrm{R}-1 \\
0-5 \\
697.5 \\
\mathrm{~V}\end{array}$ & $\begin{array}{c}\text { 956B-57R-1 } \\
25-31 \\
697.75 \\
\mathrm{~V}\end{array}$ & $\begin{array}{c}\text { 956B-57R-1 } \\
55-63 \\
698.05 \\
\mathrm{~V}\end{array}$ & $\begin{array}{c}\text { 956B-57R-1 } \\
93-101 \\
698.43 \\
\mathrm{~V}\end{array}$ \\
\hline $\begin{array}{l}\text { Major elements (wt\%) } \\
\mathrm{SiO}_{2} \\
\mathrm{TiO}_{2} \\
\mathrm{Al}_{2} \mathrm{O}_{3} \\
\mathrm{FeO} * \\
\mathrm{MnO} \\
\mathrm{MgO} \\
\mathrm{CaO} \\
\mathrm{Na}_{2} \mathrm{O} \\
\mathrm{K}_{2} \mathrm{O} \\
\mathrm{P}_{2} \mathrm{O}_{5} \\
\mathrm{LOI}\end{array}$ & $\begin{array}{r}45.20 \\
3.57 \\
10.30 \\
11.70 \\
0.16 \\
11.38 \\
7.73 \\
2.60 \\
1.37 \\
0.51 \\
4.19 \\
\end{array}$ & $\begin{array}{r}44.70 \\
3.45 \\
13.30 \\
10.44 \\
0.15 \\
6.37 \\
7.44 \\
3.74 \\
2.13 \\
0.46 \\
5.75 \\
\end{array}$ & $\begin{array}{r}45.70 \\
3.95 \\
11.50 \\
12.69 \\
0.18 \\
7.63 \\
10.60 \\
2.44 \\
0.61 \\
0.46 \\
2.63 \\
\end{array}$ & $\begin{array}{r}44.50 \\
4.82 \\
12.40 \\
12.96 \\
0.19 \\
6.37 \\
8.58 \\
3.22 \\
1.51 \\
0.62 \\
2.85 \\
\end{array}$ & $\begin{array}{r}46.20 \\
3.59 \\
9.69 \\
13.37 \\
0.16 \\
9.62 \\
10.40 \\
2.16 \\
0.78 \\
0.38 \\
1.71 \\
\end{array}$ & $\begin{array}{r}44.70 \\
3.40 \\
9.08 \\
13.41 \\
0.17 \\
10.99 \\
11.30 \\
1.84 \\
0.38 \\
0.36 \\
2.42 \\
\end{array}$ \\
\hline Total & 98.71 & 97.93 & 98.40 & 98.02 & 98.07 & 98.06 \\
\hline $\begin{array}{l}\text { Trace elements (ppm) } \\
\mathrm{V} \\
\mathrm{Cr} \\
\mathrm{Co} \\
\mathrm{Ni} \\
\mathrm{Cu} \\
\mathrm{Zn} \\
\mathrm{Rb} \\
\mathrm{Sr} \\
\mathrm{Y} \\
\mathrm{Nb} \\
\mathrm{Ba} \\
\mathrm{La} \\
\mathrm{Ce} \\
\mathrm{Zr} \\
\mathrm{Pr} \\
\mathrm{Nd}\end{array}$ & $\begin{array}{r}253 \\
298 \\
55 \\
204 \\
100 \\
100 \\
23 \\
324 \\
28 \\
45 \\
262 \\
118 \\
88 \\
310 \\
21 \\
39\end{array}$ & $\begin{array}{r}270 \\
77 \\
26 \\
79 \\
242 \\
217 \\
33 \\
797 \\
29 \\
42 \\
500 \\
96 \\
70 \\
240 \\
16 \\
35\end{array}$ & $\begin{array}{r}310 \\
728 \\
53 \\
395 \\
151 \\
125 \\
<4 \\
757 \\
33 \\
49 \\
199 \\
99 \\
88 \\
318 \\
23 \\
46\end{array}$ & $\begin{array}{r}351 \\
561 \\
48 \\
118 \\
191 \\
138 \\
10 \\
838 \\
42 \\
60 \\
432 \\
113 \\
103 \\
407 \\
23 \\
60\end{array}$ & $\begin{array}{r}275 \\
110 \\
82 \\
630 \\
127 \\
117 \\
5 \\
476 \\
29 \\
41 \\
182 \\
119 \\
51 \\
278 \\
17 \\
37\end{array}$ & $\begin{array}{r}291 \\
1081 \\
97 \\
583 \\
117 \\
125 \\
4 \\
528 \\
28 \\
36 \\
112 \\
118 \\
63 \\
240 \\
13 \\
26\end{array}$ \\
\hline $\begin{array}{l}\mathrm{Zr} / \mathrm{Nb} \\
\mathrm{Zr} / \mathrm{Y} \\
\mathrm{Nb} / \mathrm{Y}\end{array}$ & $\begin{array}{r}6.89 \\
11.07 \\
1.61\end{array}$ & $\begin{array}{l}5.71 \\
8.28 \\
1.45\end{array}$ & $\begin{array}{l}6.49 \\
9.64 \\
1.48\end{array}$ & $\begin{array}{l}6.78 \\
9.69 \\
1.43\end{array}$ & $\begin{array}{l}6.78 \\
9.59 \\
1.41\end{array}$ & $\begin{array}{l}6.67 \\
8.57 \\
1.29\end{array}$ \\
\hline
\end{tabular}


Table 6. XRF major and trace element analyses of basalt clasts, Hole 956B.

\begin{tabular}{|c|c|c|c|c|c|c|c|c|c|c|c|c|}
\hline $\begin{array}{l}\text { Hole, core, section: } \\
\text { Interval }(\mathrm{cm}): \\
\text { Depth } \\
\text { Lithologic unit } \\
\end{array}$ & $\begin{array}{c}956 \mathrm{~B}-46 \mathrm{R}-3 \\
133-148 \\
592.33 \\
\mathrm{~V} \\
\end{array}$ & $\begin{array}{c}956 \mathrm{~B}-47 \mathrm{R}-3 \\
58-64 \\
602.08 \\
\mathrm{~V} \\
\end{array}$ & $\begin{array}{c}956 \mathrm{~B}-48 \mathrm{R}-2 \\
25-42 \\
609.25 \\
\mathrm{~V} \\
\end{array}$ & $\begin{array}{c}\text { 956B-52R-1 } \\
97-106 \\
648.97 \\
\mathrm{~V} \\
\end{array}$ & $\begin{array}{c}956 \mathrm{~B}-52 \mathrm{R}-4 \\
66-70 \\
653.16 \\
\mathrm{~V} \\
\end{array}$ & $\begin{array}{c}956 \mathrm{~B}-53 \mathrm{R}-4 \\
101-111 \\
662.51 \\
\mathrm{~V} \\
\end{array}$ & $\begin{array}{c}956 \mathrm{~B}-53 \mathrm{R}-4 \\
112-125 \\
662.62 \\
\mathrm{~V} \\
\end{array}$ & $\begin{array}{c}\text { 4956B-55R-3 } \\
23-33 \\
681.23 \\
\mathrm{~V} \\
\end{array}$ & $\begin{array}{c}\text { 956B-56R-1 } \\
4-18 \\
688.54 \\
\mathrm{~V} \\
\end{array}$ & $\begin{array}{c}\text { 956B-56R-1 } \\
18-30 \\
688.68 \\
\mathrm{~V} \\
\end{array}$ & $\begin{array}{c}\text { 956B-56R-1 } \\
50-67 \\
689 \\
\mathrm{~V} \\
\end{array}$ & $\begin{array}{c}\text { 956B-56R-3 } \\
36-49 \\
691.86 \\
\mathrm{~V} \\
\end{array}$ \\
\hline $\begin{array}{l}\text { Major elements (wt } \%) \\
\mathrm{SiO}_{2} \\
\mathrm{TiO}_{2} \\
\mathrm{Al}_{2} \mathrm{O}_{3} \\
\mathrm{FeO}^{*} \\
\mathrm{MnO} \\
\mathrm{MgO} \\
\mathrm{CaO} \\
\mathrm{Na}_{2} \mathrm{O} \\
\mathrm{K}_{2} \mathrm{O} \\
\mathrm{P}_{2} \mathrm{O}_{5} \\
\mathrm{LOI}\end{array}$ & $\begin{array}{r}43.75 \\
5.50 \\
15.09 \\
10.79 \\
0.25 \\
5.49 \\
9.79 \\
3.02 \\
1.06 \\
0.55 \\
2.78 \\
\end{array}$ & $\begin{array}{r}45.32 \\
3.51 \\
11.13 \\
12.20 \\
0.17 \\
9.21 \\
11.24 \\
2.12 \\
0.54 \\
0.38 \\
2.00 \\
\end{array}$ & $\begin{array}{r}46.68 \\
3.09 \\
9.60 \\
11.54 \\
0.17 \\
11.90 \\
11.67 \\
1.72 \\
0.51 \\
0.31 \\
1.05 \\
\end{array}$ & $\begin{array}{r}45.95 \\
3.78 \\
12.23 \\
10.83 \\
0.16 \\
7.87 \\
9.16 \\
2.83 \\
1.29 \\
0.39 \\
3.60 \\
\end{array}$ & $\begin{array}{r}44.95 \\
3.98 \\
10.67 \\
12.52 \\
0.20 \\
10.10 \\
10.93 \\
1.99 \\
0.51 \\
0.45 \\
1.97 \\
\end{array}$ & $\begin{array}{r}47.80 \\
4.00 \\
13.89 \\
10.28 \\
0.17 \\
5.85 \\
10.69 \\
2.85 \\
1.24 \\
0.50 \\
1.45 \\
\end{array}$ & $\begin{array}{r}43.99 \\
4.00 \\
9.63 \\
13.28 \\
0.18 \\
10.81 \\
9.89 \\
2.06 \\
0.75 \\
0.44 \\
2.80 \\
\end{array}$ & $\begin{array}{r}45.23 \\
3.54 \\
10.26 \\
11.63 \\
0.19 \\
9.80 \\
10.51 \\
1.98 \\
1.47 \\
0.41 \\
2.86 \\
\end{array}$ & $\begin{array}{r}45.30 \\
4.32 \\
11.06 \\
12.31 \\
0.17 \\
11.05 \\
7.77 \\
2.27 \\
0.85 \\
0.50 \\
3.06 \\
\end{array}$ & $\begin{array}{r}45.51 \\
4.23 \\
10.93 \\
12.39 \\
0.17 \\
11.12 \\
7.71 \\
2.23 \\
0.89 \\
0.49 \\
2.65 \\
\end{array}$ & $\begin{array}{r}43.32 \\
4.06 \\
11.07 \\
12.53 \\
0.18 \\
10.62 \\
8.55 \\
2.13 \\
1.02 \\
0.45 \\
3.98 \\
\end{array}$ & $\begin{array}{r}44.99 \\
4.33 \\
12.41 \\
12.49 \\
0.18 \\
7.45 \\
10.05 \\
2.52 \\
0.74 \\
0.46 \\
2.61 \\
\end{array}$ \\
\hline Total & 98.07 & 97.82 & 98.24 & 98.09 & 98.27 & 98.72 & 97.83 & 97.88 & 98.66 & 98.32 & 97.91 & 98.23 \\
\hline $\begin{array}{l}\text { Trace elements (ppm) } \\
\mathrm{V} \\
\mathrm{Cr} \\
\mathrm{Co} \\
\mathrm{Ni} \\
\mathrm{Cu} \\
\mathrm{Zn} \\
\mathrm{Rb} \\
\mathrm{Sr} \\
\mathrm{Y} \\
\mathrm{Nb} \\
\mathrm{Ba} \\
\mathrm{La} \\
\mathrm{Ce} \\
\mathrm{Zr} \\
\mathrm{Pr} \\
\mathrm{Nd}\end{array}$ & $\begin{array}{r}398 \\
292 \\
38 \\
144 \\
121 \\
166 \\
9 \\
648 \\
35 \\
65 \\
306 \\
123 \\
116 \\
479 \\
24 \\
59\end{array}$ & $\begin{array}{r}315 \\
546 \\
60 \\
297 \\
148 \\
107 \\
<4 \\
562 \\
29 \\
34 \\
177 \\
106 \\
51 \\
257 \\
17 \\
31\end{array}$ & $\begin{array}{r}281 \\
779 \\
59 \\
353 \\
111 \\
103 \\
6 \\
395 \\
27 \\
29 \\
147 \\
101 \\
36 \\
217 \\
15 \\
23\end{array}$ & $\begin{array}{r}298 \\
308 \\
87 \\
179 \\
116 \\
111 \\
13 \\
564 \\
29 \\
42 \\
222 \\
93 \\
64 \\
273 \\
17 \\
42\end{array}$ & $\begin{array}{r}291 \\
919 \\
75 \\
395 \\
142 \\
124 \\
<4 \\
590 \\
31 \\
46 \\
164 \\
106 \\
90 \\
333 \\
18 \\
45\end{array}$ & $\begin{array}{r}333 \\
215 \\
50 \\
103 \\
118 \\
109 \\
12 \\
558 \\
36 \\
45 \\
244 \\
116 \\
83 \\
313 \\
19 \\
45\end{array}$ & $\begin{array}{r}299 \\
629 \\
74 \\
341 \\
97 \\
102 \\
18 \\
419 \\
31 \\
43 \\
172 \\
121 \\
74 \\
316 \\
17 \\
48\end{array}$ & $\begin{array}{r}296 \\
806 \\
71 \\
327 \\
121 \\
117 \\
14 \\
382 \\
28 \\
37 \\
269 \\
96 \\
64 \\
277 \\
18 \\
36\end{array}$ & $\begin{array}{r}274 \\
384 \\
75 \\
450 \\
94 \\
124 \\
5 \\
461 \\
34 \\
48 \\
218 \\
104 \\
79 \\
372 \\
15 \\
46\end{array}$ & $\begin{array}{r}288 \\
425 \\
62 \\
466 \\
103 \\
116 \\
8 \\
445 \\
33 \\
49 \\
202 \\
112 \\
96 \\
366 \\
18 \\
47\end{array}$ & $\begin{array}{r}296 \\
365 \\
48 \\
266 \\
109 \\
116 \\
15 \\
405 \\
31 \\
46 \\
212 \\
119 \\
73 \\
336 \\
18 \\
45\end{array}$ & $\begin{array}{r}335 \\
237 \\
76 \\
151 \\
120 \\
126 \\
4 \\
823 \\
32 \\
46 \\
193 \\
111 \\
96 \\
306 \\
21 \\
44\end{array}$ \\
\hline $\begin{array}{l}\mathrm{Zr} / \mathrm{Nb} \\
\mathrm{Zr} / \mathrm{Y} \\
\mathrm{Nb} / \mathrm{Y}\end{array}$ & $\begin{array}{r}7.37 \\
13.69 \\
1.86\end{array}$ & $\begin{array}{l}7.56 \\
8.86 \\
1.17\end{array}$ & $\begin{array}{l}7.48 \\
8.04 \\
1.07\end{array}$ & $\begin{array}{l}6.50 \\
9.41 \\
1.45\end{array}$ & $\begin{array}{r}7.24 \\
10.74 \\
1.48\end{array}$ & $\begin{array}{l}6.96 \\
8.69 \\
1.25\end{array}$ & $\begin{array}{r}7.35 \\
10.19 \\
1.39\end{array}$ & $\begin{array}{l}7.49 \\
9.89 \\
1.32\end{array}$ & $\begin{array}{r}7.75 \\
10.94 \\
1.41\end{array}$ & $\begin{array}{r}7.47 \\
11.09 \\
1.48\end{array}$ & $\begin{array}{r}7.30 \\
10.84 \\
1.48\end{array}$ & $\begin{array}{l}6.65 \\
9.56 \\
1.44\end{array}$ \\
\hline
\end{tabular}


Table 7. Electron microprobe analyses of clinopyroxene for Hole 953C.

\begin{tabular}{|c|c|c|c|c|c|c|c|c|c|c|}
\hline \multirow{2}{*}{$\begin{array}{l}\text { Sample: } \\
\text { Mineral: }\end{array}$} & \multicolumn{6}{|c|}{ 953C-90R-5, 49-55 cm } & \multirow{2}{*}{$\frac{953 \mathrm{C}-93 \mathrm{R}-4,20-22 \mathrm{~cm}}{1}$} & \multicolumn{3}{|c|}{$953 \mathrm{C}-101 \mathrm{R}-5,116-123 \mathrm{~cm}$} \\
\hline & 1 & 2 & 3 & 4 & 5 & 6 & & 1 & 2 & 3 \\
\hline $\mathrm{SiO}_{2}$ & 51.87 & 51.95 & 51.11 & 50.58 & 51.17 & 51.47 & 50.49 & 50.12 & 50.63 & 50.60 \\
\hline $\mathrm{Al}_{2} \mathrm{O}_{3}$ & 2.46 & 2.41 & 3.11 & 3.57 & 3.01 & 3.03 & 3.53 & 3.46 & 3.14 & 2.77 \\
\hline $\mathrm{TiO}_{2}$ & 1.23 & 1.23 & 1.42 & 1.64 & 1.42 & 1.33 & 1.67 & 1.66 & 1.51 & 1.42 \\
\hline $\mathrm{Cr}_{2} \mathrm{O}_{3}$ & 0.70 & 0.70 & 0.86 & 0.85 & 0.80 & 0.69 & 0.49 & 0.79 & 0.78 & 0.72 \\
\hline $\mathrm{MgO}$ & 16.96 & 17.03 & 16.41 & 16.19 & 16.34 & 16.51 & 16.01 & 16.05 & 16.41 & 16.43 \\
\hline $\mathrm{FeO}$ & 5.61 & 5.41 & 5.29 & 5.83 & 5.44 & 5.66 & 6.61 & 6.11 & 5.99 & 6.07 \\
\hline $\mathrm{MnO}$ & 0.14 & 0.09 & 0.09 & 0.09 & 0.09 & 0.07 & 0.13 & 0.09 & 0.09 & 0.11 \\
\hline $\mathrm{CaO}$ & 20.78 & 20.92 & 21.07 & 20.86 & 21.35 & 21.11 & 20.40 & 20.57 & 20.48 & 20.66 \\
\hline $\mathrm{Na}_{2} \mathrm{O}$ & 0.37 & 0.37 & 0.39 & 0.35 & 0.37 & 0.38 & 0.37 & 0.39 & 0.38 & 0.36 \\
\hline Total & 100.11 & 100.13 & 99.76 & 99.97 & 99.98 & 100.24 & 99.70 & 99.23 & 99.42 & 99.15 \\
\hline $\mathrm{Mg} \#$ & 84.3 & 84.9 & 84.7 & 83.2 & 84.3 & 83.9 & 81.2 & 82.4 & 83.0 & 82.8 \\
\hline En & 50.70 & 50.85 & 50.09 & 49.63 & 49.70 & 49.86 & 48.61 & 49.66 & 50.27 & 49.77 \\
\hline Fs & 9.56 & 9.20 & 9.67 & 10.55 & 9.49 & 9.77 & 11.77 & 10.67 & 10.35 & 10.12 \\
\hline Wo & 39.73 & 39.94 & 40.23 & 39.82 & 40.80 & 40.36 & 39.62 & 39.66 & 39.37 & 40.11 \\
\hline
\end{tabular}

Table 8. Electron microprobe analyses of clinopyroxene for Hole 954B.

\begin{tabular}{lrrr}
\hline \multirow{2}{*}{ Sample: } & \multicolumn{3}{c}{$954 \mathrm{~B}-35 \mathrm{R}-2,1-11 \mathrm{~cm}$} \\
\cline { 2 - 4 } Mineral: & \multicolumn{1}{c}{1} & \multicolumn{1}{c}{2} & \multicolumn{1}{c}{3} \\
\hline $\mathrm{SiO}_{2}$ & 51.85 & 51.28 & 52.09 \\
$\mathrm{Al}_{2} \mathrm{O}_{3}$ & 2.08 & 2.13 & 1.60 \\
$\mathrm{TiO}_{2}$ & 0.95 & 0.96 & 0.79 \\
$\mathrm{Cr}_{2} \mathrm{O}_{3}$ & 0.79 & 0.80 & 0.56 \\
$\mathrm{MgO}$ & 16.74 & 16.84 & 17.08 \\
$\mathrm{FeO}$ & 4.57 & 4.58 & 4.39 \\
$\mathrm{MnO}$ & 0.07 & 0.07 & 0.04 \\
$\mathrm{CaO}$ & 22.01 & 22.02 & 22.22 \\
$\mathrm{Na} 2$ & 0.33 & 0.34 & 0.30 \\
$\mathrm{Total}$ & 99.40 & 99.04 & 99.08 \\
$\mathrm{Mg \#}$ & 86.71 & 86.77 & 87.39 \\
$\mathrm{En}$ & 50.11 & 50.06 & 49.79 \\
$\mathrm{Fs}$ & 7.42 & 7.29 & 7.09 \\
$\mathrm{Wo}$ & 42.47 & 42.65 & 43.12 \\
\hline
\end{tabular}

\section{Hole 954B}

Unit IV (Sections 157-954B-35R-1 through 39R-2, 408-446 mbsf) is a 38-m-thick dark green to gray basaltic breccia. The structureless very poorly sorted breccia appears to represent a single thick depositional unit. Large basalt fragments, both matrix- and clast-supported, consist mostly of nonvesicular aphyric basalts and moderately vesicular and nonvesicular olivine-clinopyroxene- and minor plagioclasephyric basalts. Vesicular clasts are more abundant than nonvesicular ones. In Core 157-954B-34R, an unusual clast of dark gray, mafic tuff contains accretionary lapilli up to $5 \mathrm{~mm}$ in diameter.

\section{Hole 956B}

Unit V (Sections 157-956B-43R-3 through 57R-1, 564.1-704 mbsf) is $140 \mathrm{~m}$ thick and consists mostly of hyaloclastite tuff, lapillistone, and basaltic breccia with minor interbeds of nannofossil claystone with foraminifers (Fig. 3). We have subdivided Unit V into 12 subunits comprising four thinner units of massive to bedded epiclastic basaltic sandstones, four units of fine-grained hyaloclastites, and three thicker, strongly graded debris-flow deposits.

Thick debris-flow deposits (Subunits 5 [interval 157-956B-43R$6,37.5 \mathrm{~cm}$ to $45 \mathrm{R}-1,7.5 \mathrm{~cm}$ ], 9 [157-956B-45R-4, $52 \mathrm{~cm}$, to 48R-3, $26 \mathrm{~cm}$ ] and 12 [157-956B-49R-1, $26 \mathrm{~cm}$, to $57 \mathrm{R}-1,121 \mathrm{~cm}])$. The debris-flow deposits strongly increase in grain size and in the abundance of matrix toward the base. The maximum size of the angular to subangular basalt clasts at the base is $\sim 20 \mathrm{~cm}$. The proportion of oxidized vesicular basalt clasts in Subunit 12 decreases significantly from $\sim 5 \%$ near the base to $<1 \%$ at the top. The matrix consists of dark brown clay, nannofossil clay and unfilled planktonic foraminifers. The two thinner upper debris-flow deposits (Subunits 5 and 9) con- tain a higher proportion of angular to vesicular former sideromelane shards and, in Subunit 5, nonvesicular drop-shaped lapilli (Fig. 6), whose glassy cores are still fresh. The thick basal debris flow in contrast contains almost no former sideromelane shards. Nonvesicular lapilli compose $\sim 60 \%-75 \%$ and vesicular lapilli $\sim 25 \%-40 \%$ of the clasts. Aphyric clasts are dominant in the upper part of Subunit 9, but pyroxene-phyric and olivine- (altered) phyric clasts dominate in the lower part (Core 157-956B-46R).

Basaltic sandstones (Subunits 1 [interval 157-956B-43R-3, $93 \mathrm{~cm}$, to $43 \mathrm{R}-4,70 \mathrm{~cm}$ ], 6 [interval $157-956 \mathrm{~B}-45 \mathrm{R}-1,75 \mathrm{~cm}$, to $45 \mathrm{R}-1,101$ $\mathrm{cm}$ ], 8 [interval $157-956 \mathrm{~B}-45 \mathrm{R}-3,64 \mathrm{~cm}$, to $45 \mathrm{R}-4,37 \mathrm{~cm}$ ] and 10 [interval $157-956 \mathrm{~B}-48 \mathrm{R}-3,33 \mathrm{~cm}$, to $48 \mathrm{R}-3,123 \mathrm{~cm}]$ ), generally $<1 \mathrm{~m}$ thick, show weak grading and contain very little matrix (nannofossil foraminifer ooze) with filled planktonic foraminifers. Clasts are angular to subangular and bedding, lamination, and cross-bedding are common. Zeolite cement fills the pores in many of these sandstones. These rocks are interpreted to represent dominantly erosional debris.

Hyaloclastite tuffs (Subunits 2 [interval 157-956B-43R-4, $89 \mathrm{~cm}$, to $43 \mathrm{R}-5,21 \mathrm{~cm}$ ], 3 [interval $157-956 \mathrm{~B}-43 \mathrm{R}-5,50 \mathrm{~cm}$, to $43 \mathrm{R}-5,75$ $\mathrm{cm}$ ], 4 [interval 157-956B-43R-6, $15 \mathrm{~cm}$, to 43R-6, $25 \mathrm{~cm}$ ] and 7 [interval $157-956 \mathrm{~B}-45 \mathrm{R}-2,0 \mathrm{~cm}$, to $45 \mathrm{R}-2,43 \mathrm{~cm}$ ) consist mostly of angular nonvesicular to pumiceous mafic shards (Fig. 24) in which the sideromelane has been completely replaced by smectite. These are hyaloclastite tuffs proper.

\section{DISCUSSION}

\section{Fragmentation Processes}

Three fragmentation processes were postulated by Schmincke and von Rad (1979) based on particle characteristics to explain the volcaniclastic sediments drilled at Site 397. These processes include submarine pyroclastic eruptions, accompanied at shallow water by steam explosions, to produce sideromelane shards, entry of lava flows into the sea, and subaerial erosion. We are adding two additional processes of fragmentation: collapse of the submarine and/or subaerial flank of the island volcano and collapse of lava deltas.

\section{Submarine Eruptions}

The moderate to high vesicularity (15-90 vol\%) of many ash- and lapilli-sized formerly glassy particles in the deposits of Units VI and VII (Hole 953C) and in the upper hyaloclastite deposits of Hole 956B (Sections 157-953C-48R-3 through 43R-3) is important evidence for moderate to shallow water $(<<\sim 500 \mathrm{~m})$ eruptive processes, representing the late seamount stage. Hyaloclastites composed dominantly of nonvesicular shards of the type encountered as mass-flow deposits at Site 397 (Schmincke and von Rad, 1979) are restricted to the lower 
Table 9. Electron microprobe analyses of clinopyroxene for Hole 956B.

\begin{tabular}{|c|c|c|c|c|c|c|c|c|c|c|c|c|c|c|c|c|c|c|}
\hline \multirow{2}{*}{$\begin{array}{l}\text { Sample: } \\
\text { Mineral: }\end{array}$} & \multicolumn{2}{|c|}{ 956B-47R-2, $102-110 \mathrm{~cm}$} & \multicolumn{2}{|c|}{ 956B-47R-3, $26-31 \mathrm{~cm}$} & \multicolumn{3}{|c|}{ 956B-48R-2, 25-42 cm } & \multicolumn{3}{|c|}{ 956B-53R-2, $18-24 \mathrm{~cm}$} & \multicolumn{2}{|c|}{ 956B-53R-4, $112-125 \mathrm{~cm}$} & \multicolumn{3}{|c|}{$956 \mathrm{~B}-54 \mathrm{R}-2,58-65 \mathrm{~cm}$} & \multicolumn{2}{|c|}{ 956B-56R-2, 89-103 cm } & \multirow{2}{*}{$\frac{956 \mathrm{~B}-56 \mathrm{R}-3,69-77 \mathrm{~cm}}{1}$} \\
\hline & 1 & 2 & 1 & 2 & 1 & 2 & 3 & 1 & 2 & 3 & 1 & 2 & 1 & 2 & 3 & 1 & 2 & \\
\hline $\mathrm{SiO}_{2}$ & 49.78 & 51.45 & 50.31 & 49.75 & 50.56 & 50.88 & 51.46 & 50.45 & 50.92 & 49.78 & 48.51 & 49.47 & 50.48 & 49.59 & 52.44 & 49.16 & 48.89 & 49.64 \\
\hline $\mathrm{Al}_{2} \mathrm{O}_{3}$ & 3.85 & 2.65 & 3.18 & 3.67 & 3.53 & 3.25 & 2.60 & 3.25 & 2.85 & 3.76 & 4.64 & 3.48 & 3.03 & 3.38 & 1.56 & 3.75 & 3.93 & 3.30 \\
\hline $\mathrm{TiO}_{2}$ & 1.65 & 1.17 & 1.64 & 1.90 & 1.43 & 1.45 & 1.20 & 1.59 & 1.35 & 1.75 & 2.28 & 1.68 & 1.49 & 1.71 & 0.82 & 1.83 & 1.94 & 1.64 \\
\hline $\mathrm{Cr}_{2} \mathrm{O}_{3}$ & 0.39 & 0.39 & 0.40 & 0.42 & 0.33 & 0.35 & 0.19 & 0.57 & 0.62 & 0.62 & 0.24 & 0.40 & 0.43 & 0.21 & 0.38 & 0.40 & 0.27 & 0.21 \\
\hline $\begin{array}{ll}\mathrm{MgO}_{2} \\
\mathrm{Cu}\end{array}$ & 15.64 & 16.29 & 15.81 & 15.47 & 15.26 & 15.41 & 16.00 & 15.68 & 16.38 & 15.50 & 14.51 & 15.26 & 15.99 & 15.15 & 16.84 & 15.38 & 15.00 & 15.21 \\
\hline $\mathrm{FeO}$ & $\begin{array}{r}15.04 \\
6.33\end{array}$ & $\begin{array}{r}10.29 \\
5.37\end{array}$ & $\begin{array}{r}6.01 \\
6.08\end{array}$ & $\begin{array}{r}6.58 \\
6.58\end{array}$ & $\begin{array}{r}15.30 \\
6.34\end{array}$ & $\begin{array}{r}15.41 \\
6.27\end{array}$ & $\begin{array}{r}6.13 \\
6.13\end{array}$ & $\begin{array}{r}15.00 \\
6.45\end{array}$ & $\begin{array}{r}10.50 \\
6.38\end{array}$ & 6.66 & 7.31 & $\begin{array}{r}15.20 \\
6.52\end{array}$ & 6.25 & 6.62 & $\begin{array}{r}0.04 \\
4.76\end{array}$ & $\begin{array}{r}6.00 \\
6.09\end{array}$ & 6.72 & 7.33 \\
\hline $\mathrm{MnO}$ & $\begin{array}{l}0.33 \\
0.10\end{array}$ & 0.09 & $\begin{array}{l}0.08 \\
0.08\end{array}$ & $\begin{array}{l}0.08 \\
0.10\end{array}$ & $\begin{array}{l}0.34 \\
0.12\end{array}$ & $\begin{array}{l}0.27 \\
0.11\end{array}$ & $\begin{array}{l}0.13 \\
0.11\end{array}$ & $\begin{array}{l}0.43 \\
0.10\end{array}$ & $\begin{array}{l}0.30 \\
0.10\end{array}$ & $\begin{array}{l}0.00 \\
0.11\end{array}$ & 0.11 & $\begin{array}{l}0.52 \\
0.10\end{array}$ & $\begin{array}{l}0.25 \\
0.11\end{array}$ & $\begin{array}{l}0.02 \\
0.10\end{array}$ & $\begin{array}{l}4.10 \\
0.10\end{array}$ & $\begin{array}{l}0.09 \\
0.07\end{array}$ & $\begin{array}{l}0.12 \\
0.10\end{array}$ & $\begin{array}{l}7.33 \\
0.16\end{array}$ \\
\hline $\begin{array}{l}\mathrm{MnOO} \\
\mathrm{CaO}\end{array}$ & $\begin{array}{l}0.10 \\
21.67\end{array}$ & $\begin{array}{r}0.09 \\
22.30\end{array}$ & $\begin{array}{r}0.08 \\
21.75\end{array}$ & $\begin{array}{l}0.10 \\
21.30\end{array}$ & $\begin{array}{l}0.12 \\
22.04\end{array}$ & $\begin{array}{r}0.11 \\
21.94\end{array}$ & $\begin{array}{l}0.11 \\
21.94\end{array}$ & $\begin{array}{r}0.10 \\
20.92\end{array}$ & $\begin{array}{l}0.10 \\
20.23\end{array}$ & $\begin{array}{l}0.111 \\
20.65\end{array}$ & $\begin{array}{l}0.11 \\
21.27\end{array}$ & $\begin{array}{l}0.10 \\
21.81\end{array}$ & $\begin{array}{l}0.11 \\
21.41\end{array}$ & $\begin{array}{l}0.10 \\
22.27\end{array}$ & $\begin{array}{l}0.10 \\
22.58\end{array}$ & 21.51 & $\begin{array}{l}0.10 \\
21.32\end{array}$ & $\begin{array}{r}0.10 \\
20.80\end{array}$ \\
\hline $\mathrm{Na}_{2} \mathrm{O}$ & $\begin{array}{r}0.07 \\
0.45\end{array}$ & 0.35 & 0.37 & $\begin{array}{r}1.50 \\
0.41 \\
\end{array}$ & $\begin{array}{r}0.04 \\
0.39 \\
\end{array}$ & $\begin{array}{r}1.94 \\
0.38 \\
\end{array}$ & $\begin{array}{r}1.94 \\
0.36 \\
\end{array}$ & $\begin{array}{r}20.92 \\
0.45 \\
\end{array}$ & $\begin{array}{r}20.25 \\
0.44 \\
\end{array}$ & $\begin{array}{r}20.05 \\
0.47 \\
\end{array}$ & $\begin{array}{r}0.49 \\
\end{array}$ & $\begin{array}{r}1.01 \\
0.45 \\
\end{array}$ & $\begin{array}{r}21.41 \\
0.46 \\
\end{array}$ & $\begin{array}{r}0.40 \\
\end{array}$ & $\begin{array}{r}0.30 \\
0.32 \\
\end{array}$ & $\begin{array}{r}1.31 \\
0.40 \\
\end{array}$ & $\begin{array}{r}0.41 \\
0.41\end{array}$ & $\begin{array}{r}20.00 \\
0.39 \\
\end{array}$ \\
\hline Total & 99.86 & 100.06 & 99.62 & 99.61 & 100.00 & 100.05 & 99.99 & 99.46 & 99.29 & 99.30 & 99.36 & 99.16 & 99.65 & 99.44 & 99.80 & 98.59 & 98.57 & 98.68 \\
\hline Mg\# & 81.49 & 84.39 & 82.24 & 80.74 & 81.10 & 81.41 & 82.31 & 81.24 & 82.06 & 80.56 & 77.95 & 80.67 & 82.02 & 80.31 & 86.30 & 81.83 & 79.91 & 78.71 \\
\hline En & & 48.12 & $\begin{array}{l}47.43 \\
47.24\end{array}$ & $\begin{array}{l}47.25 \\
47 . / 4\end{array}$ & $\begin{array}{l}46.46 \\
4\end{array}$ & $\begin{array}{l}1.41 \\
46.62\end{array}$ & $\begin{array}{l}47.06 \\
47.31\end{array}$ & $\begin{array}{l}48.23 \\
48.23\end{array}$ & $\begin{array}{l}49.94 \\
49.00\end{array}$ & 48.28 & 45.27 & 46.23 & $\begin{array}{l}47.94 \\
47.02\end{array}$ & 45.11 & 48.54 & $\begin{array}{l}47.24 \\
47.23\end{array}$ & 46.17 & 46.22 \\
\hline $\begin{array}{l}\mathrm{Cll} \\
\mathrm{Fs}\end{array}$ & 8.48 & $\begin{array}{r}40.12 \\
7.50\end{array}$ & 9.46 & 10.31 & $\begin{array}{r}40.40 \\
9.95\end{array}$ & $\begin{array}{l}40.02 \\
10.39\end{array}$ & $\begin{array}{r}4.00 \\
9.02\end{array}$ & $\begin{array}{l}11.13 \\
11\end{array}$ & 10.72 & $\begin{array}{l}40.20 \\
11.34\end{array}$ & 11.70 & 9.63 & 9.40 & $\begin{array}{r}5.11 \\
9.78\end{array}$ & $\begin{array}{r}40.04 \\
7.51\end{array}$ & 9.05 & $\begin{array}{l}40.17 \\
10.25\end{array}$ & $\begin{array}{l}40.22 \\
11.42\end{array}$ \\
\hline Wo & 43.16 & 44.39 & 43.12 & 42.44 & 43.60 & 42.99 & 43.92 & 40.64 & 39.35 & 40.38 & 43.03 & 44.14 & 42.66 & 45.11 & 43.95 & 43.71 & 43.58 & 42.36 \\
\hline
\end{tabular}

Table 9 (continued).

\begin{tabular}{|c|c|c|c|c|}
\hline \multirow{2}{*}{$\begin{array}{l}\text { Sample: } \\
\text { Mineral: }\end{array}$} & \multicolumn{2}{|c|}{ 956B-57R-1, 0-5 cm } & \multicolumn{2}{|c|}{ 956B-57R-1, 93-101 cm } \\
\hline & 1 & 2 & 1 & 2 \\
\hline $\mathrm{SiO}_{2}$ & 52.30 & 50.26 & 51.38 & 50.63 \\
\hline $\mathrm{Al}_{2} \mathrm{O}_{3}$ & 1.81 & 2.96 & 2.12 & 2.70 \\
\hline $\mathrm{TiO}_{2}$ & 0.78 & 1.53 & 0.90 & 1.12 \\
\hline $\mathrm{Cr}_{2} \mathrm{O}_{3}$ & 0.77 & 0.18 & 0.81 & 0.54 \\
\hline $\mathrm{MgO}$ & 17.38 & 15.69 & 16.52 & 15.94 \\
\hline $\mathrm{FeO}$ & 4.61 & 7.56 & 4.77 & 5.58 \\
\hline $\mathrm{MnO}$ & 0.07 & 0.15 & 0.06 & 0.09 \\
\hline $\mathrm{CaO}$ & 21.24 & 20.27 & 22.35 & 22.11 \\
\hline $\mathrm{Na}_{2} \mathrm{O}$ & 0.34 & 0.41 & 0.37 & 0.38 \\
\hline Total & 99.30 & 99.01 & 99.28 & 99.09 \\
\hline Mg\# & 87.06 & 78.72 & 86.06 & 83.60 \\
\hline En & 51.68 & 47.15 & 49.15 & 47.71 \\
\hline Fs & 7.33 & 11.80 & 7.39 & 7.86 \\
\hline Wo & 40.98 & 41.05 & 43.47 & 44.43 \\
\hline
\end{tabular}


Table 10. Electron microprobe analyses of olivine for Hole 953C.

\begin{tabular}{|c|c|c|c|c|c|c|c|c|}
\hline \multirow{2}{*}{$\begin{array}{l}\text { Sample: } \\
\text { Mineral: }\end{array}$} & \multicolumn{2}{|c|}{ 953C-90R-5, 49-55 cm } & \multicolumn{3}{|c|}{ 953C-93R-4, 20-22 cm } & \multicolumn{3}{|c|}{$953 \mathrm{C}-101 \mathrm{R}-5,116-123 \mathrm{~cm}$} \\
\hline & 1 & 2 & 1 & 2 & 3 & 1 & 2 & 3 \\
\hline $\mathrm{SiO}_{2}$ & 38.84 & 38.66 & 39.51 & 39.85 & 39.89 & 38.97 & 39.30 & 38.54 \\
\hline $\mathrm{MgO}$ & 43.05 & 42.75 & 44.97 & 44.67 & 44.304 & 43.87 & 44.46 & 42.52 \\
\hline $\mathrm{FeO}$ & 17.19 & 17.64 & 13.84 & 14.73 & 15.481 & 16.43 & 15.58 & 18.07 \\
\hline $\mathrm{MnO}$ & 0.26 & 0.24 & 0.18 & 0.18 & 0.24 & 0.22 & 0.22 & 0.25 \\
\hline $\mathrm{CaO}$ & 0.31 & 0.33 & 0.23 & 0.30 & 0.33 & 0.32 & 0.28 & 0.31 \\
\hline $\mathrm{Na}_{2} \mathrm{O}$ & 0.12 & 0.11 & 0.12 & 0.11 & 0.12 & 0.12 & 0.12 & 0.12 \\
\hline $\mathrm{NiO}$ & 0.28 & 0.29 & 0.38 & 0.29 & 0.27 & 0.31 & 0.31 & 0.25 \\
\hline Total & 100.05 & 100.02 & 99.23 & 100.14 & 100.62 & 100.24 & 100.26 & 100.06 \\
\hline Fo & 81.70 & 81.21 & 85.28 & 84.39 & 83.61 & 82.64 & 83.57 & 80.75 \\
\hline $\mathrm{Fa}$ & 18.30 & 18.79 & 14.72 & 15.61 & 16.39 & 17.36 & 16.43 & 19.25 \\
\hline
\end{tabular}

Table 11. Electron microprobe analyses of olivine for Hole 954B

\begin{tabular}{lrr}
\hline Sample: & \multicolumn{2}{c}{$954 \mathrm{~B}-37 \mathrm{R}-1,137-140 \mathrm{~cm}$} \\
\cline { 2 - 3 } Mineral: & \multicolumn{1}{c}{1} & \multicolumn{1}{c}{2} \\
\hline $\mathrm{SiO}_{2}$ & 38.44 & 38.46 \\
$\mathrm{MgO}$ & 40.10 & 40.03 \\
$\mathrm{FeO}$ & 20.33 & 20.52 \\
$\mathrm{MnO}$ & 0.27 & 0.28 \\
$\mathrm{CaO}$ & 0.26 & 0.27 \\
$\mathrm{Na} 2$ & 0.13 & 0.11 \\
$\mathrm{NiO}$ & 0.23 & 0.23 \\
$\mathrm{Total}$ & 99.76 & 99.90 \\
$\mathrm{Fo}$ & 77.86 & 77.67 \\
$\mathrm{Fa}$ & 22.14 & 22.33 \\
\end{tabular}

portions of the drilled section at Hole 953C (Cores 157-953C-85R through 103R).

\section{Entry of Lava Flows into the Sea, and the Collapse of Lava Deltas}

Lava deltas grow along the coast of active volcanic islands where lava flows enter the sea and build inherently unstable platforms and deltas that episodically collapse. A wide variety of particles forms at the interface, depending on the flow rate of the lava, its viscosity, subaerial, and submarine slope gradients, and other factors. Breccias form by sliding or collapse of the submarine flow-foot breccia during or shortly after the deposition of lava units. Such processes occur at present along the southeast coast of Kilauea volcano, Hawaii (Tribble, 1991). Collapse, gradual sinking and subsidence of the entire lava delta or large blocks of it, caused by the load of the newly accumulating lavas and breccias of the delta, counteract the constructional processes.

Coarse, heterolithologic, clast- and matrix-supported, poorly sorted breccias composed of angular and rounded fragments of subaerial and pillow lava with hyaloclastite and foraminifer/nannofossil ooze or noncalcareous clay matrix are common in the sections drilled. The polymict clast assemblage, abundance of vesicular and oxidized clasts, and subrounded nature of many fragments suggests a subaerial volcanic source, but hyaloclastite fragments and clasts of pillow basalts are evidence that the breccias are a mixture of subaerially and shallow-water derived volcanics, as they typically occur during the emergent phase of volcanic islands. Such deposits are especially characteristic of lithologic Unit VI (Hole 953C) and lithologic Subunits 5 and 9 (Hole 956B). They are interpreted as a mixture of fragments of different origin, including those formed by fragmentation of subaerial lava flows along the shore line as they entered the sea, possibly from collapse of near-shore unstable lava platforms and submarine foreset deltas seaward of a platform, and by subaerial erosion.

The upper part of Section 157-953C-71R-4, strongly graded between 40 and $0 \mathrm{~cm}$, contains $\sim 30 \%$ of light greenish angular large shards and small lapilli, probably altered sideromelane fragments.
Table 12. Electron microprobe analyses of olivine for Hole 956B.

\begin{tabular}{|c|c|c|}
\hline Sample: & 956B-48R-2, 25-42 cm & 956B-56R-2, 89-103 cm \\
\hline Mineral: & 1 & 1 \\
\hline $\mathrm{SiO}_{2}$ & 39.07 & 39.44 \\
\hline $\mathrm{MgO}$ & 42.39 & 42.79 \\
\hline $\mathrm{FeO}$ & 17.29 & 17.52 \\
\hline $\mathrm{MnO}$ & 0.24 & 0.26 \\
\hline $\mathrm{CaO}$ & 0.24 & 0.34 \\
\hline $\mathrm{NiO}$ & 0.24 & 0.26 \\
\hline Total & 99.48 & 100.61 \\
\hline $\begin{array}{l}\text { Fo } \\
\text { Fa }\end{array}$ & $\begin{array}{l}81.38 \\
18.62\end{array}$ & $\begin{array}{l}81.32 \\
18.68\end{array}$ \\
\hline
\end{tabular}

This coarse-grained, very strongly graded debrite may be related to a lava flow that entered the sea. The occurrence of drop-shaped, glassy, nonvesicular lapilli in the 10-m-thick uppermost debris flow at Hole 956B (Subunit 5) suggests that volcanic activity, possibly lava flows entering the sea, were instrumental in triggering these debris flows. The abundance of coarse biogenic skeletal debris is further evidence for the derivation of these materials from the near-shore shallowwater environment.

\section{Erosion}

The occurrence of oxidized and weathered pebbles, especially in the coarser breccias (from $888 \mathrm{~m}$ to $927 \mathrm{~m}$ at Hole $953 \mathrm{C}$, all of the 38 $\mathrm{m}$ at Hole 954B and Subunit 12 at Hole 956B), and of subrounded to rounded fragments of crystalline basalt, indicates that subaerial volcanism and hence subaerial erosion were already taking place while the most voluminous volcanic activity was still shallow submarine.

The tachylitic particles in the thin basaltic turbidites encountered chiefly at Hole 953C (lithologic Unit V), but also at Hole 956B between Subunit 5 and the P1 tuff, are interpreted to have formed dominantly by subaerial erosional fragmentation of scoria cone lapilli and lava flows because of the coexistence with crystallized basalt fragments and the oxidized nature of many fragments. The occurrence of altered nonvesicular blocky sideromelane shards in some of these turbidites may be related to quenching during episodic entry of lava flows into the sea. Formerly glassy shards may also have become mixed with the subaerial erosional detritus during single or multistage transport down the submarine flanks of the island.

\section{Flank Collapse}

Submarine volcaniclastic deposits representing unequivocal flank collapse events have not yet been characterized well in the literature. We argue that three major breccia bodies that range in minimum thickness from $22 \mathrm{~m}$ (Subunit 9, Hole 956B) through $40 \mathrm{~m}$ (Hole 
Table 13. Electron microprobe analysis of plagioclase, Hole 954B and Hole 956B.

\begin{tabular}{|c|c|c|c|c|c|c|c|}
\hline \multirow{2}{*}{$\begin{array}{l}\text { Sample: } \\
\text { Mineral: }\end{array}$} & \multicolumn{2}{|c|}{ 954B-35R-2, 30-34 cm } & \multirow{2}{*}{$\frac{954 \mathrm{~B}-37 \mathrm{R}-1,79-83 \mathrm{~cm}}{1}$} & \multicolumn{3}{|c|}{ 956B-44R-4, 110-124 cm } & \multirow{2}{*}{$\frac{956 \mathrm{~B}-56 \mathrm{R}-3,69-77 \mathrm{~cm}}{1}$} \\
\hline & 1 & 2 & & 1 & 2 & 3 & \\
\hline $\mathrm{SiO}_{2}$ & 51.47 & 52.37 & 52.32 & 53.34 & 53.34 & 53.84 & 51.04 \\
\hline $\mathrm{TiO}_{2}^{2}$ & 0.15 & 0.19 & 0.21 & 0.13 & 0.15 & 0.16 & 0.16 \\
\hline $\mathrm{Al}_{2} \mathrm{O}_{3}$ & 29.24 & 28.57 & 28.87 & 28.34 & 28.39 & 28.11 & 29.41 \\
\hline $\mathrm{FeO}$ & 0.75 & 0.85 & 0.61 & 0.82 & 0.77 & 0.73 & 0.68 \\
\hline $\mathrm{BaO}$ & 0.01 & 0.03 & 0.06 & 0.05 & 0.03 & 0.06 & 0.02 \\
\hline $\mathrm{CaO}$ & 12.80 & 12.26 & 12.02 & 11.61 & 11.65 & 11.19 & 13.01 \\
\hline $\mathrm{Na}_{2} \mathrm{O}$ & 3.65 & 3.81 & 3.96 & 4.14 & 4.13 & 4.31 & 3.43 \\
\hline $\mathrm{K}_{2} \mathrm{O}$ & 0.27 & 0.31 & 0.42 & 0.36 & 0.40 & 0.39 & 0.28 \\
\hline Total & 98.45 & 98.52 & 98.58 & 98.86 & 98.92 & 98.88 & 98.14 \\
\hline
\end{tabular}

Table 14. Electron microprobe analyses of amphibole, Hole 956B.

\begin{tabular}{|c|c|c|c|c|c|c|c|c|c|c|c|c|c|c|c|c|c|}
\hline \multirow{2}{*}{$\begin{array}{l}\text { Sample: } \\
\text { Mineral: }\end{array}$} & \multicolumn{3}{|c|}{ 956B-43R-4, 40-54 cm } & \multicolumn{3}{|c|}{ 956B-45R-CC, 7-14 cm } & \multicolumn{3}{|c|}{ 956B-46R-1, $14-20 \mathrm{~cm}$} & \multicolumn{3}{|c|}{ 956B-46R-1, 117-130 cm } & \multicolumn{2}{|c|}{ 956B-46R-2, 62-81 cm } & \multicolumn{3}{|c|}{ 956B-46R-3, 25-37 cm } \\
\hline & 1 & 2 & 3 & 1 & 2 & 3 & 1 & 2 & 3 & 1 & 2 & 3 & 1 & 2 & 1 & 2 & 3 \\
\hline $\mathrm{SiO}_{2}$ & 46.74 & 46.06 & 46.18 & 46.70 & 46.61 & 45.72 & 46.08 & 46.39 & 45.70 & 45.94 & 47.05 & 46.76 & 47.33 & 47.10 & 46.74 & 47.09 & 46.44 \\
\hline $\mathrm{TiO}_{2}$ & 1.63 & 1.63 & 1.80 & 1.46 & 1.71 & 1.91 & 1.05 & 1.85 & 1.83 & 2.15 & 1.60 & 1.74 & 1.42 & 1.70 & 1.91 & 1.76 & 1.72 \\
\hline $\mathrm{Al}_{2} \mathrm{O}_{3}$ & 7.76 & 7.55 & 7.85 & 7.68 & 7.97 & 8.77 & 8.47 & 7.95 & 8.55 & 8.45 & 7.61 & 7.90 & 7.19 & 7.09 & 7.83 & 7.44 & 8.08 \\
\hline $\mathrm{FeO}$ & 9.59 & 12.68 & 9.61 & 9.52 & 9.38 & 9.66 & 9.74 & 9.40 & 9.54 & 9.78 & 9.13 & 9.15 & 9.95 & 9.78 & 9.12 & 9.10 & 9.28 \\
\hline $\mathrm{MnO}$ & 0.27 & 0.60 & 0.30 & 0.25 & 0.30 & 0.13 & 0.37 & 0.19 & 0.28 & 0.15 & 0.29 & 0.28 & 0.25 & 0.27 & 0.26 & 0.29 & 0.26 \\
\hline $\mathrm{MgO}$ & 17.08 & 14.65 & 16.73 & 16.95 & 16.92 & 16.48 & 16.48 & 16.82 & 16.47 & 16.53 & 17.36 & 17.00 & 16.61 & 16.59 & 17.00 & 17.12 & 16.91 \\
\hline $\mathrm{CaO}$ & 9.10 & 8.15 & 9.31 & 9.52 & 9.38 & 9.81 & 9.48 & 9.54 & 9.98 & 9.41 & 9.48 & 9.59 & 8.54 & 8.51 & 9.01 & 9.19 & 9.46 \\
\hline $\mathrm{Na}_{2} \mathrm{O}$ & 4.06 & 4.29 & 3.96 & 3.95 & 3.88 & 3.41 & 4.24 & 3.95 & 3.53 & 3.62 & 3.91 & 3.80 & 4.25 & 4.31 & 4.08 & 4.06 & 3.83 \\
\hline $\mathrm{K}_{2} \mathrm{O}$ & 0.66 & 0.62 & 0.63 & 0.61 & 0.61 & 0.90 & 0.45 & 0.70 & 0.87 & 0.78 & 0.67 & 0.69 & 0.63 & 0.61 & 0.64 & 0.58 & 0.71 \\
\hline $\mathrm{F}$ & 0.15 & 0.13 & 0.13 & 0.13 & 0.13 & 0.18 & 0.08 & 0.17 & 0.19 & 0.13 & 0.14 & 0.18 & 0.12 & 0.12 & 0.16 & 0.16 & 0.14 \\
\hline $\mathrm{Cr}_{2} \mathrm{O}_{3}$ & 0.04 & 0.00 & 0.06 & 0.01 & 0.09 & 0.05 & 0.08 & 0.02 & 0.05 & 0.02 & 0.07 & 0.02 & 0.02 & 0.00 & 0.11 & 0.03 & 0.00 \\
\hline Total & 97.94 & 97.35 & 97.36 & 97.54 & 97.79 & 97.83 & 97.26 & 97.68 & 97.67 & 97.80 & 98.13 & 97.86 & 97.21 & 96.88 & 97.64 & 97.56 & 97.60 \\
\hline
\end{tabular}

954B) and $75 \mathrm{~m}$ (Subunit 12, Hole 956B) and are very strongly sizegraded (Hole 956B) represent the collapse of probably both subaerial and submarine flanks of Gran Canaria. Evidence is based mainly on the extremely wide range in size, shape and crystallinity of the dominantly angular basalt clasts and the dominance of fragments of probably subaerially erupted basaltic lava and scoria deposits. The abundance of clayey matrix and of large rip up clasts of pelagic sediment scattered within the deposits, contrasting strongly with the relatively well-sorted dominantly hyaloclastic debris-flow deposits of Unit VII at Site 953, further argues for very large volume collapse events and intense scouring of soft flank and basin sediments.

The basal, strongly graded debris-flow deposit at Hole 956B is characterized by the abundance of brown matrix, which appears to be a mixture of noncalcareous clay and nannofossil ooze with very minor scattered planktonic generally open foraminifers set in a matrix of coarse sand- to lapilli-sized, exclusively basaltic fragments that range extremely widely in shape, crystallinity, and size. The poorly sorted lapillistones at the base of the debris-flow deposit of Subunit 9 (Hole 956B), rich in brownish matrix, resemble very much those of the thick Subunit 12 . There is no evidence from the petrography that the 75-m-thick basal debris flow was triggered by volcanic activity directly and the diverse petrography of the clasts also suggests a compositionally heterogeneous source. We tentatively postulate that flank collapse of the shield volcano involved both subaerial and submarine portions judging from the broad spectrum of basaltic clasts. Such a mechanism might also help to explain the abundance of matrix especially in the lower part of this deposit. We think the matrix represents clay deposited on the lower flank of the seamount. The matrix was not derived from shallow-water biogenic sediments because of the absence of coarse-grained neritic skeletal debris and the unfilled nature of the planktonic foraminifers, all of which appear to be of the type with very delicate skeletons. These unconsolidated sediments may have either become mobilized from the collapse of the underlying submarine part of the large slump or, possibly more likely, became incorporated as the very large debris flow raced down the lower flanks of the volcano and probably eroded deeply into the soft sediments.

There is circumstantial evidence that this flow may correlate exactly in time with the large scar of the Horgazales Basin that was carved into the shield basalt during the late stage of the basaltic shield building activity prior to eruption of the more evolved aphyric and plagioclase-phyric Horgazales basalts (Schmincke, 1968). The scar was still a morphological basin during eruption of the Mogán ignimbrites which attain their maximum thickness $(>400 \mathrm{~m})$ in the Horgazales area. Not only the timing would correlate but also the change from pyroxene- and olivine-dominated basalts (the older Guigui Formation from the base of the collapse scar), whereas the younger Horgazales more evolved and plagioclase-rich basalts would be correlated with the two upper thinner debris-flow deposits (Subunits 5 and 9). In fact, the steep cliffs (the scar is up to $400 \mathrm{~m}$ deep) might well have promoted waterfall-like activity of lava flows draining into the sea and thus explain the glassy drop-like lapilli found in the uppermost debris flow (Subunit 5) at Hole 956B.

The basalt breccia at Site 954, thought to represent the collapsed and mixed flank deposits of the Gran Canaria shield, sits directly atop sediments dated as $10 \mathrm{Ma}$. No volcaniclastic sediments corresponding to the Fataga or Mogán phase on Gran Canaria are present between lithologic Units III and IV, indicating that there is a large as yet unexplained hiatus between $\sim 10.7$ and $\sim 14 \mathrm{Ma}$. The lithologic and time gap was interpreted (Schmincke, Weaver, Firth et al., 1995) as being caused by slumping of the interval representing the Fataga and Mogán volcaniclastic sediments and associated biogenic sediments. Funck and Lykke-Anderson (Chap. 2, this volume) interpreted the basalt breccia as part of a slump block that overlies Mogán and Fataga age sediments. Unit IV (Hole 954B) probably represents a breccia emplaced at $14 \mathrm{Ma}$ after completion of the basaltic shield, but prior to the Mogán phase because it completely lacks rhyolites and trachyphonolites of the Mogán and Fataga Formations that are several hundred meters thick at the north coast. If the collapse had taken place after $14 \mathrm{Ma}$ as suggested by Funck and Lykke-Anderson (Chap. 2, 


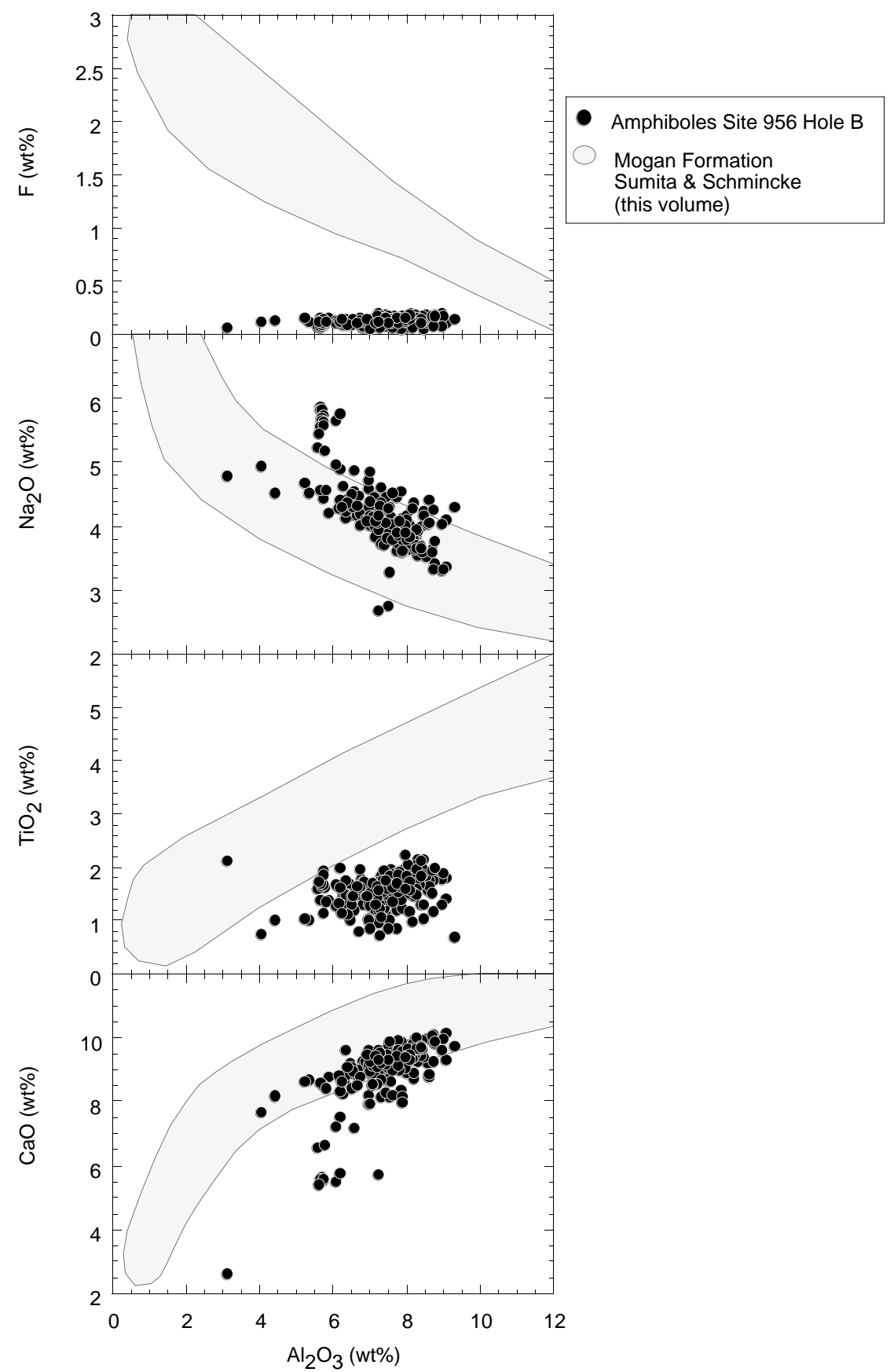

Figure 15. Amphibole composition of Hole 956B.

this volume) the breccia should contain fragments of Mogán and especially Fataga rocks.

\section{Transport Processes}

We distinguish several modes of transport based on the grain size and sedimentary structures of the basaltic volcaniclastic rocks.

\section{Debris-Flow Deposits}

Most prominent are strongly graded debris-flow deposits up to 75 $\mathrm{m}$ thick (Pl. 4, Figs. 1-4) in which variably rounded and vesicular basalt clasts are concentrated in the lower $\sim 10 \%-20 \%$ of a deposit. There is a general positive correlation between maximum clast size, Md and thickness of a debris-flow deposit. The bulk is composed of strongly size-graded massive lapillistone to coarse tuff and the top
$5 \%-10 \%$ or so shows laminar bedding to local cross-bedding of sandsized material representing the more dilute portion above the main coarse-grained bed load.

From a sedimentologic point of view, the strong grading with extreme clast enrichment at the base is similar in the debris-flow deposits at Sites 956 and 954. The contrast between the Hole 956B debrites and the breccias from Hole 954B is the rather massive nongraded nature of the lapillistones and breccias at Hole 954B, possibly because these breccias represent the proximal facies which, if drilled further away from the island such as Site 956, might have developed more pronounced grading.

There is a strong contrast in the degree of scouring which is very pronounced at Site 956 - as reflected in the abundance of clay matrix and common pickup sediment clasts within the main breccia body (Pl. 4, Figs. 5, 6) - and the apparent absence for evidence of scouring in the matrix-poor hyaloclastite debris-flow deposits in the 190-m- 

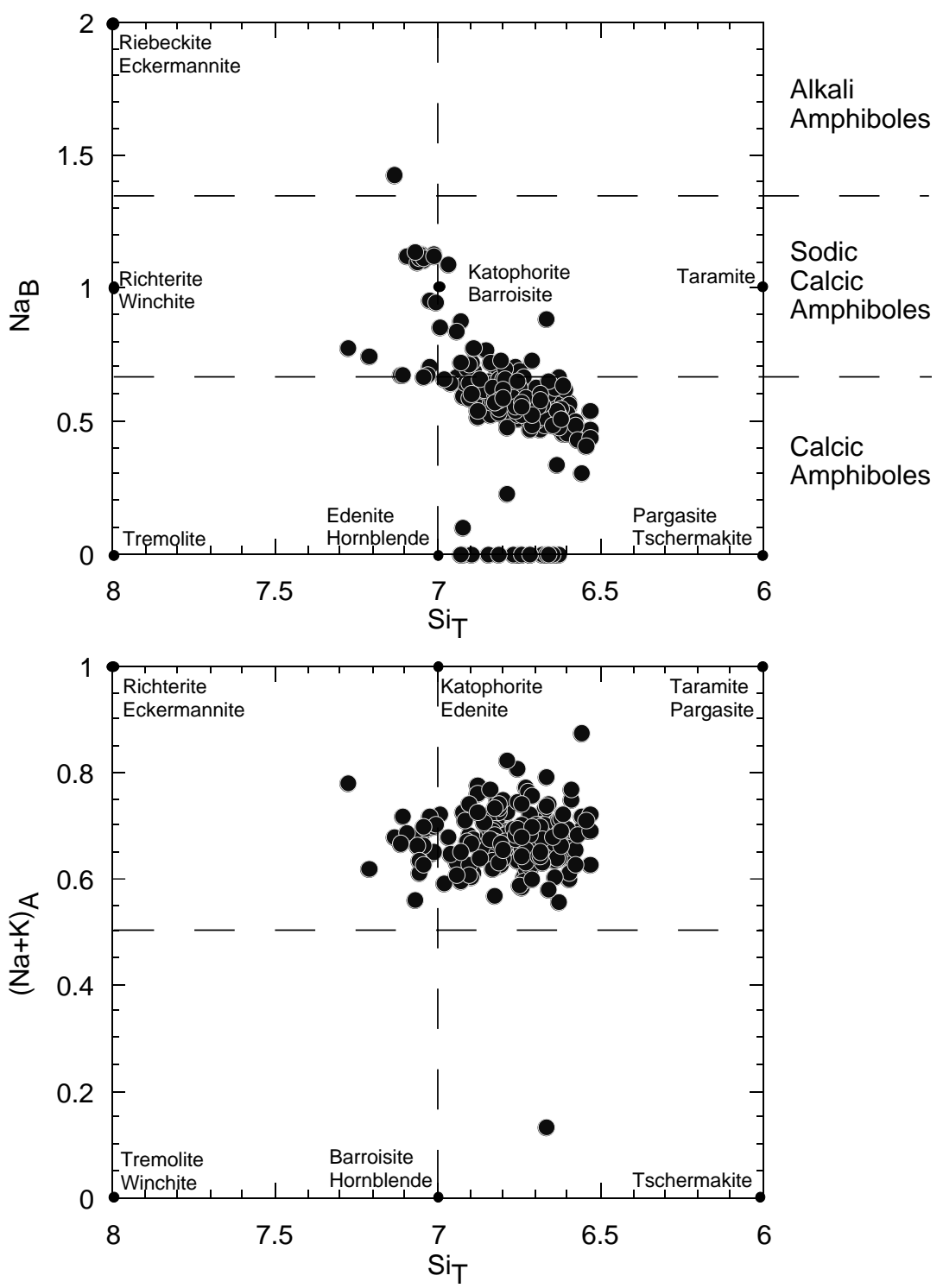

\section{A, B and T: Cations sites in the standard formula \\ Hole 956B}

Classification of amphiboles of Site 956B, having $(\mathrm{Ca}+\mathrm{Na})_{B} \geq 1.34$

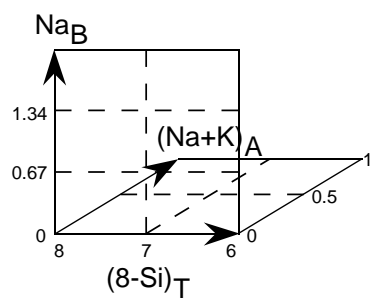

Figure 15 (continued).

thick lithologic Unit VII in Site 953. Part of the contrast may be because of the very high sedimentation rate at Site 953, where practically no pelagic interbeds or mixed sediment turbidites occur between the $\sim 16$ debris-flow units. In other words, the substrate between successive debris flows consisted of sand-sized hyaloclastites.

\section{Turbidites}

The volcaniclastic sandstones and siltstones, principally those of lithologic Unit V (Hole 953C) and those above Subunits 5 and 9 (Hole 956B), are interpreted as turbidites principally based on the sharp basal contacts, pronounced grading, and small grain size. A wide spectrum of massive to bedded or cross-bedded basal parts may reflect a corresponding range from highly dilute turbidites to concentrated bed loads.
The upper 5\%-15\% of debrites, where recovered, especially those of lithologic Unit VII (Hole 953C), resemble some turbidites in their grain-size distribution. This suggests that some or most turbidites represent the distal facies of debris flows. Moreover, at Site 953, where the sediment transport down the submarine flank of the volcano is envisioned to have been more channeled than at Site 956, the hundreds of turbidites of Unit V may represent the overbank facies away from a main channel area where the parent debris flow may have been transported.

\section{Source Areas for the Hyaloclastite Tuffs and Debrites}

The fact that the hyaloclastites and largely epiclastic sandstones at Sites 953, 955, and 956 are found directly below P1 tuffs is unequivocal evidence that at least the uppermost deposits of the basaltic 


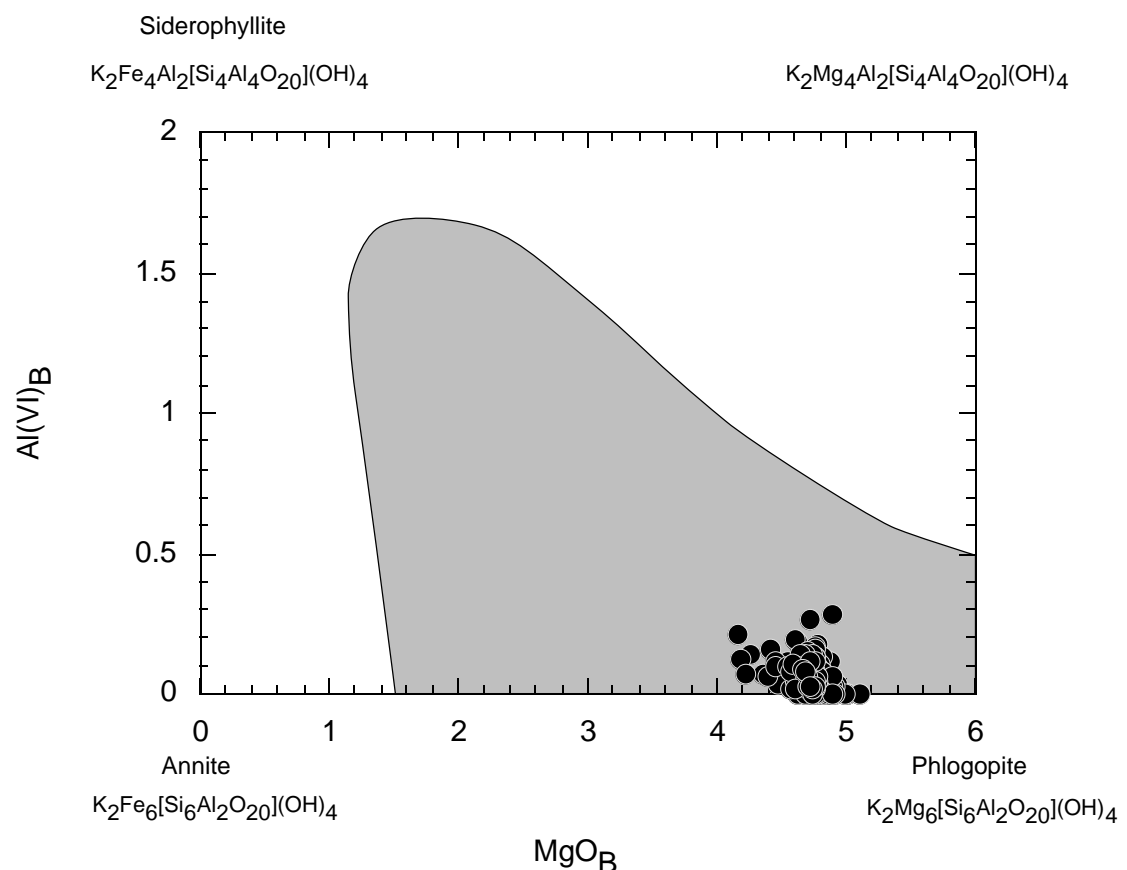

A, B: Cations sites in the standard formula
- Hole $956 \mathrm{~B}$
Figure 16. Mica composition of Hole 956B. The principal components of biotite compositions and the field (gray) in which most natural biotites lie (after Deer, Howie, and Zussman, 1993). volcaniclastic intervals (the tachylite/lithic clast-dominated Unit $\mathrm{V}$ at Hole 953C and Subunit I of Unit V at Hole 956B) were derived from the seamount precursor to Gran Canaria Island and the subaerial shield phase. Moreover, the facies succession in Sites 953 and 956, which indicates a general shallowing - based on the increasing vesicularity of sideromelane shards - and transition to subaerial fragmentation with decreasing age is strong evidence that the entire hyaloclastite sections at all sites were derived from Gran Canaria.

In Hole 953C, Units VI and VII are separated from each other by $\sim 2 \mathrm{~m}$ of calcareous claystone at 966 to $968 \mathrm{mbsf}$. Seismic data are interpreted as suggesting that Unit VII thickens eastward possibly indicating derivation from Fuerteventura (Funck and Lykke-Anderson, Chap. 2, this volume). The similarity of this debris-flow facies to that making up part of lithologic Unit V at Hole 956B (Subunit 5 and hyaloclastite turbidites), however, is taken by us as evidence for Gran Canaria as the source area. The 2-m-thick nonvolcanic interval merely suggests a longer period of nonvolcanic sedimentation, in concert with the other sites where discontinuous sedimentation is the rule.

The basaltic volcaniclastic debris-flow deposits at DSDP Site 397 had been originally related to Fuerteventura as source area, based largely on the then prevalent interpretation that the shield basalts of Gran Canaria were younger than $14 \mathrm{Ma}$., and the tracing of a widespread reflector to Fuerteventura, believed to be equivalent to these lapillistones (Schmincke and von Rad, 1979). The fact that the entire submarine and subaerial construction of the basaltic shield phase of Gran Canaria may have lasted as much as 2 m.y. for these deposits as well does not exclude Gran Canaria as a source area for the DSDP Site 397 debris either. The upper volcaniclastic mass-flow deposit at Site 397 may represent the distal facies of a major debris-flow deposit related to a huge flank collapse of Gran Canaria.

The uppermost basaltic volcaniclastics drilled at sites 953 and 956 underlie the submarine P1 tuffs and were thus deposited immediately prior to $\mathrm{P} 1$ at $14.0 \mathrm{Ma}$. The entire basaltic volcaniclastic sections drilled at both sites may have been laid down during a time period that probably did not exceed $1 \mathrm{~m} . \mathrm{y}$. and possibly much less. The major differences between both sections reflect contrasts between the age of the shield basalts in eastern vs. southern/southwestern Gran Canaria. In eastern Gran Canaria, the shield basalt series is highly dissected by canyons up to several hundred meters deep, such as the major Barranco de Tirajana. The basalts are dominantly clinopyroxene-olivine-phyric, plagioclase being rare as a phenocryst phase. Aphyric trachybasalts (mugearites) occur as clasts in local fanglomerates, sandstones, and siltstones of the Barranco de Balos Formation that fill canyons in the eastern shield basalts laid down prior to eruption of the Mogán ignimbrites. In contrast, the basal P1 ignimbrite throughout southern and southwestern Gran Canaria almost conformably overlies plagioclase-phyric and more evolved aphyric basalts of the Horgazales Formation that are as much as $200 \mathrm{~m}$ thick. This indicates that the subaerial shield basalts of Gran Canaria presently exposed formed at least two major volcanic edifices. No dominantly plagioclase-phyric basalts are known to have erupted from the Aguimes volcano to the east. This volcano was being highly dissected while volcanic activity built a younger volcano to the west. We do not know the age differences between the two shield volcanoes nor do we know whether an entirely new volcano was built from the seafloor in western Gran Canaria or whether only the uppermost sequences of the western edifice are younger than the Aguimes series.

The three characteristic lithologies at Hole 953C, the turbidites of Unit $\mathrm{V}$ and the breccias of Unit VI are practically missing at Hole 956B. Conversely, the upper basaltic volcaniclastics at Hole 956B differ appreciably in their lithology from those at Hole $953 \mathrm{C}$ as do the "placer sands" described above. Biostratigraphic correlation of the sections is impossible. We tentatively suggest the following correlation: Basaltic turbidites of Unit V at Hole 953C which we believe reflect erosion of a shield volcano in eastern Gran Canaria that was either inactive or beyond the peak of main activity are tentatively correlated in time with the trachybasaltic uppermost sequence at Hole 956B. This correlation is largely based on the interpretation of the evolution of the shield basalt sequence on the island itself. Organicrich pelagic sediments and turbidites in lithologic Unit V (Hole 953C) begin in Section 157-953C-71R-3 and become more prominent in Section 157-953C-70R-6. In other words, the rate and tempo 

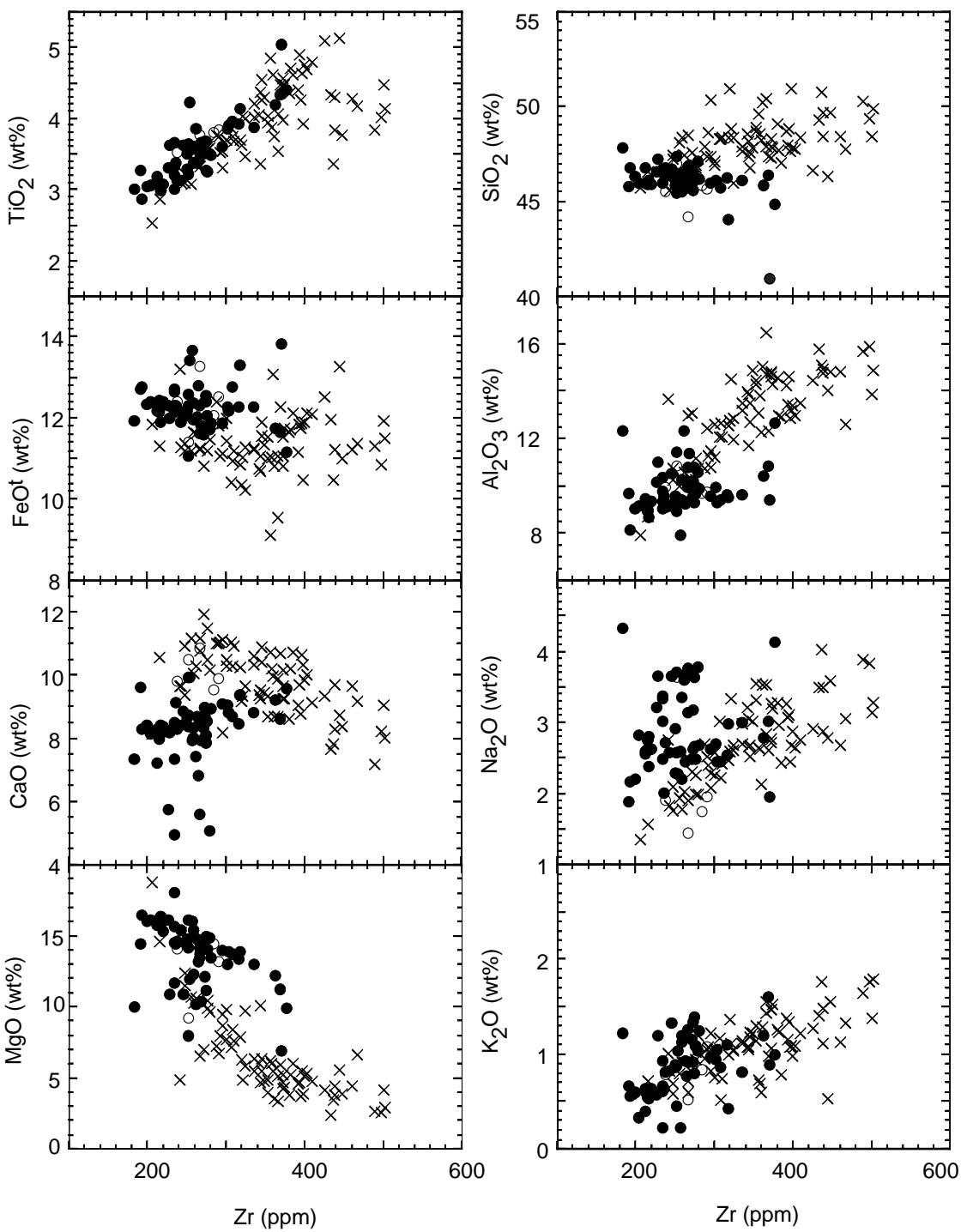

Figure 17. Major element concentration vs. $\mathrm{Zr}$ (ppm) for volcaniclastic sediments and single basalt clasts (Sections 157-953C-73R-4 through 103R-6).

- Volcaniclastic sediments in Hole 953C

- Basalt clasts in Hole 953C

$\times$ Subaerial shield basalts (Heuschkel, 1996) of brownish volcaniclastic turbidite deposition decreases within Core 157-953C-71R until deposition of ash P1. Apparently, the supply rate of volcaniclastic particles gradually decreased at Site 953 prior to eruption of ignimbrite $\mathrm{P} 1$. The turbidites, that is, the sand portion in Core 157-953C-70R and the upper part of 71R. contain much more abundant foraminifers than turbidites further down, also indicating that the island had become more mature in the east and that the influx of volcaniclastic material had decreased. The abundance of turbidites of brownish, fine-grained basaltic material probably records the ongoing activity of the island as well as erosion.

\section{Exotic Sandstone}

The source area for the exotic sandstones in Subunit 11, Sections 157-956B-48R-123 through 49R-1 (Fig. 25) is difficult to reconstruct. The extremely high concentration of nonvolcanic heavy minerals in these placer-like sands suggests a sudden influx from a nearby alkalic evolved to mafic/ultramafic plutonic source. Syenites and mafic/ultramafic plutonic rocks containing abundant amphibole, biotite, sphene, apatite, and minor Cr-spinel have been described from the basal complexes of both Fuerteventura (Munoz, 1969) and La Gomera (Cendrero, 1969). The syenites on Fuerteventura have been dated at $20 \mathrm{Ma}$. (Coello et al., 1992) and the basal complex of La Gomera has yielded a similar age (Cantagrel et al., 1984). Fuerteventura and probably La Gomera therefore were already in existence when Gran Canaria grew as a seamount and later an island.

Because La Gomera is closer to the drill site $(118 \mathrm{~km})$ than the basal complex of Fuerteventura, and that Gran Canaria may have been above sea level at the time of deposition of the "placer sands," and that the characteristic minerals of the "placer suite" have not been found in the $\sim 300$-m-thick hyaloclastites at Hole 953C, we suggest La Gomera as the most likely source. We postulate that erosion of syenitic to ultramafic rocks on La Gomera had produced placer-like beach sands on the eastern slopes of the island which were remobilized and carried by currents to the area of Hole 956B. This remobilization was possibly triggered by the generation and emplacement of 

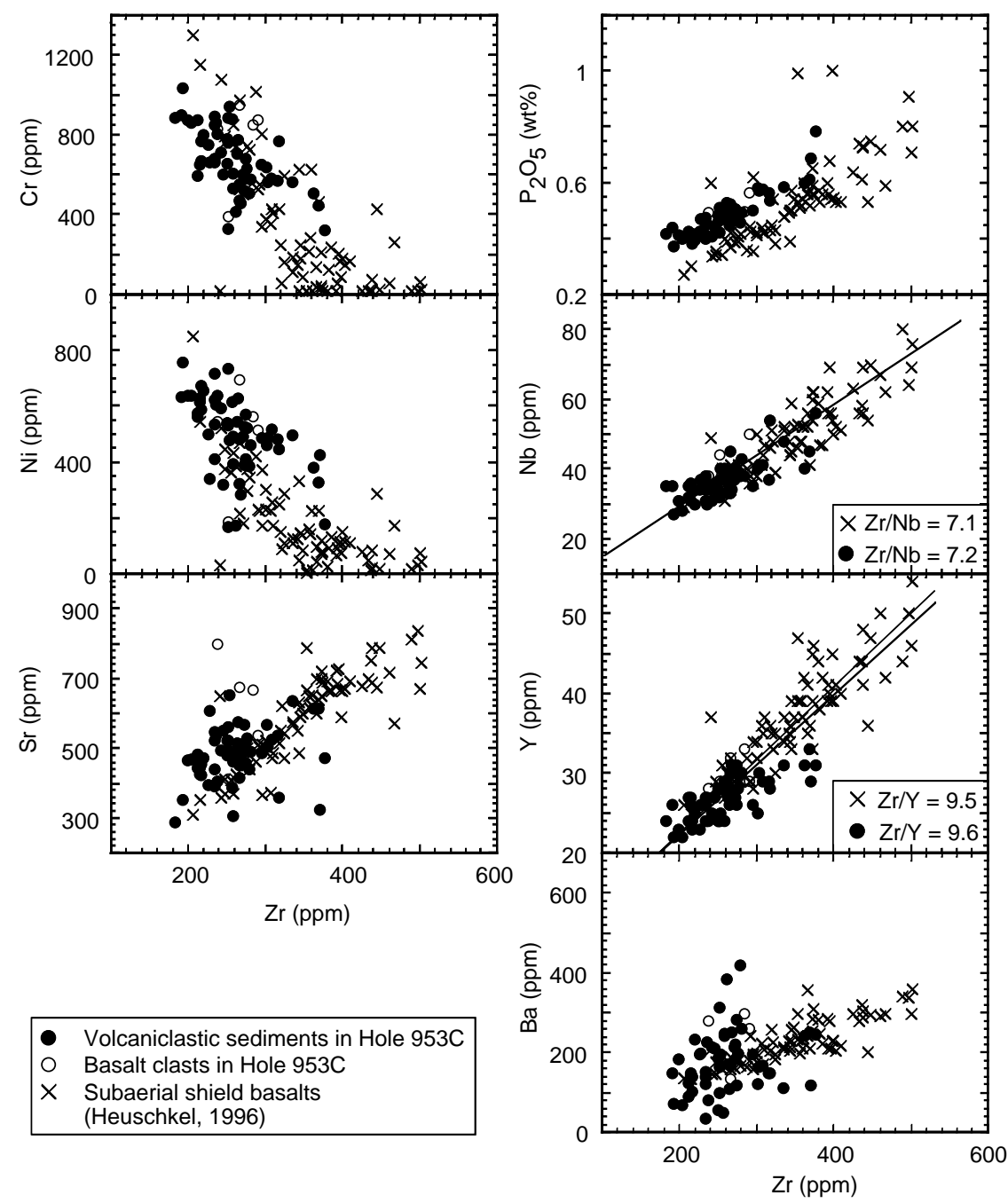

Basalt clasts in Hole 953C

$\times$ Subaerial shield basalts (Heuschkel, 1996)
Figure 17 (continued). Trace element concentration vs. $\mathrm{Zr}(\mathrm{ppm})$ for volcaniclastic sediments and single basalt clasts (Sections 157-953C-73R-4 through 103R-6). the huge underlying debris flow that was drilled between 629 and 704 $\mathrm{m}$, the base having not been penetrated. This 75- to 100 - $\mathrm{m}$-thick (?) flow may have triggered a tsunami, possibly related to collapse of the submarine/subaerial flank of Gran Canaria that also created the large Horgazales Basin in southwestern Gran Canaria as discussed above (Fig. 20). The "placer sands" were most likely deposited even higher up on the slope of Gran Canaria because scattered phlogopite crystals occur in the younger debris flows as well. These debris flows could have eroded upslope flanks during their passage down the submarine slopes of the island, phlogopite flakes being carried in the suspended masses.

We cannot exclude that plutonic complexes occur in the core of Gran Canaria itself and were the source areas for these "placer sands." Abundant fragments of mid-ocean ridge basalt-type tholeiitic basalts and gabbros occur in the fanglomerates of the Barranco de Balos Formation sandwiched between the Aguimes Formation of the shield basalts and the Mogán ignimbrites, indicating that Jurassic oceanic crustal material-possibly transported to the surface by phreatic explosions - had become available for erosion on Gran Canaria. No in situ plutonic rocks have been found so far on Gran Canaria, however, so we tentatively exclude Gran Canaria as likely source for the "placer sands" of Hole 956B.

The common occurrence of phlogopite flakes-the "xenocryst vagabond" (Sumita and Schmincke, Chap. 15, this volume)-not only through the uppermost basaltic sands and turbidites at Hole 956B but also Hole 953C as well as the basal few tens of meters into the evolved tephra series, might also be explained plausibly by the scouring action of coarse-grained turbidites that descended the slope of Gran Canaria seamount. The slowly settling micas are easily mobilized and suspended by each successive flow.

\section{ACKNOWLEDGMENTS}

We are grateful to the Deutsche Forschungsgemeinschaft (Grants Schm 250/41, L0-1, 62) and STEAM (MAS2-CT94-0083) for financial support. We thank M. Sumita for her help with the figures and plates.

\section{REFERENCES}

Cantagrel, J.-M., Cendrero, A., Fúster, J.-M., Ibarrola, E., and Jamond, C., 1984. K-Ar-chronology of the volcanic eruptions in the Canarian Archipelago: Island of La Gomera. Bull. Volcanol., 47:597-609.

Cendrero, A., 1969. The volcano-plutonic complex of La Gomera. Bull. Volcanol., 34:537-561.

Coello, J., Cantagrel, J.-M., Hernín, F., Fúster, J.-M., Ibarrola, E., Ancochea, E., Casquet, C., Jamond, C., Díaz de Téran, J.-R., and Cendrero, A., 1992. Evolution of the eastern volcanic ridge of the Canary Island based on new K-Ar data. J. Volcanol. Geotherm. Res., 53:251-274.

Deer, W.A., Howie, R.A., and Zussman, J., 1993. An Introduction to the Rock-Forming Minerals: London (Longman). 
Freundt, A., and Schmincke, H.-U., 1992. Mixing of rhyolite, trachyte and basalt magma erupted from a vertically and laterally zoned reservoir, composite flow P1, Gran Canaria. Contrib. Mineral. Petrol., 112:1-19. 1995. Petrogenesis of rhyolite-trachyte-basalt composite ignimbrite P1, Gran Canaria, Canary Islands. J. Geophys. Res., 100:455-474.

Funck, T., 1996. Structure of the volcanic apron north of Gran Canaria deduced from reflection seismic, bathymetric and borehole data [Ph.D. dissert.]. Univ. Kiel.

Heuschkel, S., 1996. Die miozänen Schildbasalte von Gran Canaria: Stratigraphische und geochemische Untersuchungen zum Aufbau und zur Entwicklung einer ozeanischen Vulkaninsel im Atlantik [Ph.D. dissert.]. ChristianAlbrechts-Univ., Kiel, Federal Republic of Germany.

Jarosewich, E., Nelen, J.A., and Norberg, J.A., 1980. Reference samples for electron microprobe analysis. Geostand. Newsl., 4:43-47.

Jarosewich, E., Nelen, J.A., and Norberg, J.A., 1980. Reference samples for electron microprobe analysis. Geostand. Newsl., 4:43-47.

Metrich, N., and Clocchiatti, C., 1989. Melt inclusion investigation of the volatile behaviour in historic alkali basaltic magmas of Etna. Bull. Volcanol., 51:185-198.

Mosbah, M., Metrich, N., and Massiot, P., 1991. PIGME fluorine determination using a nuclear microprobe with application to glass inclusions. Nucl. Instr. Meth. Phys. Res., B58:227-231.

Munoz, G., 1969. Estudio petrol—gico de las formaciones alcalinas de Fuerteventura (Islas Canarias). Estud. Geol., 25:257-310.
1976. Ring complexes of Pajara in Fuerteventura Island. Bull. Volcanol., 33:840-861.

Schmincke, H.-U., 1968. Faulting versus erosion and the reconstruction of the Mid-Miocene shield volcano of Gran Canaria. Geol. Mitt., 8:23-50.

, 1982. Volcanic and chemical evolution of the Canary Islands. In von Rad, U., Hinz, K., Sarnthein, M., and Seibold, E. (Eds.), Geology of the Northwest African Continental Margin: Berlin (Springer), 273-306.

Schmincke, H.-U., and von Rad, U., 1979. Neogene evolution of Canary Island volcanism inferred from ash layers and volcaniclastic sandstones of DSDP Site 397 (Leg 47A). In von Rad, U., Ryan, W.B.F., et al., Init. Repts. DSDP, 47 (Pt. 1): Washington (U.S. Govt. Printing Office), 703725.

Schmincke, H.-U., Weaver, P.P.E., Firth, J.V., et al., 1995. Proc. ODP, Init. Repts., 157: College Station, TX (Ocean Drilling Program).

Staudigel, H., and Schmincke, H.-U., 1984. The Pliocene seamount series of La Palma/Canary Islands. J. Geophys. Res., 89:11195-11215.

Tribble, G.W., 1991. Underwater observations of active lava flows from Kilauea volcano, Hawaii. Geology 19:633-636.

Date of initial receipt: 3 July 1996

Date of acceptance: 22 January 1997

Ms 157SR-110

Table 15. Electron microprobe analyses of mica, Hole 956B.

\begin{tabular}{|c|c|c|c|c|c|c|c|c|c|c|c|c|c|c|c|}
\hline \multirow{2}{*}{$\begin{array}{l}\text { Sample: } \\
\text { Mineral: }\end{array}$} & \multicolumn{5}{|c|}{ 956-45R-CC, 7-14 cm } & \multicolumn{5}{|c|}{ 956B-46R-1, 14-20 cm } & \multicolumn{5}{|c|}{$956 \mathrm{~B}-46 \mathrm{R}-1,117-130 \mathrm{~cm}$} \\
\hline & 1 & 2 & 3 & 4 & 5 & 1 & 2 & 3 & 4 & 5 & 1 & 2 & 3 & 4 & 5 \\
\hline $\mathrm{SiO}_{2}$ & 39.32 & 39.55 & 39.54 & 39.45 & 39.37 & 39.48 & 39.49 & 38.95 & 39.22 & 39.38 & 39.24 & 39.43 & 38.64 & 39.46 & 39.16 \\
\hline $\mathrm{TiO}_{2}$ & 1.74 & 1,57 & 1.56 & 2.00 & 1.74 & 2.08 & 1.86 & 1.86 & 2.03 & 2.01 & 1.73 & 1.71 & 1.90 & 2.02 & 1.87 \\
\hline $\mathrm{Al}_{2} \mathrm{O}_{3}$ & 13.29 & 13.51 & 13.38 & 13.40 & 13.65 & 13.71 & 13.64 & 13.90 & 13.76 & 13.47 & 13.73 & 13.75 & 14.07 & 13.43 & 13.79 \\
\hline $\mathrm{FeO}$ & 9.39 & 9.54 & 9.50 & 9.64 & 9.58 & 9.72 & 9.67 & 10.41 & 9.60 & 9.58 & 9.88 & 9.48 & 11.26 & 9.44 & 9.46 \\
\hline $\mathrm{MnO}$ & 0.12 & 0.14 & 0.15 & 0.10 & 0.12 & 0.11 & 0.12 & 0.1 & 5 & 0.12 & 0.12 & 0.14 & 0.15 & 0.12 & 0.13 \\
\hline $\mathrm{MgO}$ & 22.04 & 21.89 & 22.30 & 22.30 & 21.94 & 21.90 & 21.70 & 21.74 & 20.94 & 21.68 & 21.79 & 21.86 & 22.25 & 20.33 & 22.04 \\
\hline $\mathrm{Na}_{2} \mathrm{O}$ & 1.87 & 1.78 & 1.95 & 1.88 & 1.75 & 1.68 & 1.81 & 1.64 & 1.70 & 1.77 & 1.70 & 1.51 & 1.73 & 1.77 & 1.71 \\
\hline $\mathrm{K}_{2} \mathrm{O}$ & 7.18 & 7.22 & 7.05 & 6.88 & 7.28 & 7.44 & 7.35 & 7.52 & 7.31 & 7.28 & 6.83 & 7.53 & 7.06 & 7.34 & 7.10 \\
\hline $\mathrm{Cr}_{2} \mathrm{O}_{3}$ & 0.09 & 0.03 & 0.02 & 0.07 & 0.04 & 0.05 & 0.04 & 0.04 & 0.03 & 0.02 & 0.07 & 0.02 & 0.03 & 0.03 & 0.03 \\
\hline & 0.17 & 0.19 & 0.15 & 0.18 & 0.17 & 0.17 & 0.15 & 0.16 & 0.19 & 0.18 & 0.17 & 0.16 & 0.12 & 0.20 & 0.19 \\
\hline Total & 95.45 & 95.42 & 95.62 & 95.54 & 95.61 & 96.16 & 95.88 & 95.58 & 95.66 & 95.63 & 95.36 & 96.01 & 95.34 & 95.87 & 95.15 \\
\hline
\end{tabular}

Table 15 (continued).

\begin{tabular}{|c|c|c|c|c|c|c|c|c|c|}
\hline \multirow{2}{*}{$\begin{array}{l}\text { Sample: } \\
\text { Mineral: }\end{array}$} & \multicolumn{5}{|c|}{ 956B-46R-2, 62-81 cm } & \multicolumn{4}{|c|}{ 956B-46R-3, 25-37 cm } \\
\hline & 1 & 2 & 3 & 4 & 5 & 1 & 2 & 3 & 4 \\
\hline $\mathrm{SiO}_{2}$ & 38.80 & 39.36 & 39.47 & 39.09 & 39.35 & 39.29 & 39.41 & 40.27 & 39.09 \\
\hline $\mathrm{TiO}_{2}$ & 1.96 & 1.87 & 1.76 & 2.07 & 1.80 & 1.98 & 1.80 & 1.80 & 2.03 \\
\hline $\mathrm{Al}_{2} \mathrm{O}_{3}$ & 13.94 & 13.76 & 13.56 & 13.78 & 13.46 & 13.50 & 13.46 & 13.06 & 13.86 \\
\hline $\mathrm{FeO}$ & 10.01 & 9.57 & 9.38 & 9.52 & 9.33 & 9.32 & 9.29 & 9.10 & 9.67 \\
\hline $\mathrm{MnO}$ & 0.17 & 0.14 & 0.13 & 0.10 & 0.18 & 0.10 & 0.10 & 0.13 & 0.10 \\
\hline $\mathrm{MgO}$ & 21.71 & 21.54 & 21.74 & 22.03 & 21.46 & 21.98 & 22.19 & 22.31 & 21.73 \\
\hline $\mathrm{Na}_{2} \mathrm{O}$ & 1.67 & 1.88 & 1.89 & 1.72 & 1.92 & 1.72 & 1.83 & 1.86 & 1.63 \\
\hline $\mathrm{K}_{2} \mathrm{O}$ & 7.29 & 7.29 & 7.15 & 7.24 & 7.18 & 7.18 & 6.89 & 7.34 & 6.85 \\
\hline $\mathrm{Cr}_{2} \mathrm{O}_{3}$ & 0.04 & 0.04 & 0.02 & 0.02 & 0.03 & 0.05 & & & \\
\hline & 0.17 & 0.17 & 0.15 & 0.18 & 0.15 & 0.18 & 0.17 & 0.18 & 0.19 \\
\hline Total & 95.63 & 95.80 & 95.56 & 95.20 & 95.37 & 95.03 & 95.61 & 95.70 & 95.74 \\
\hline
\end{tabular}



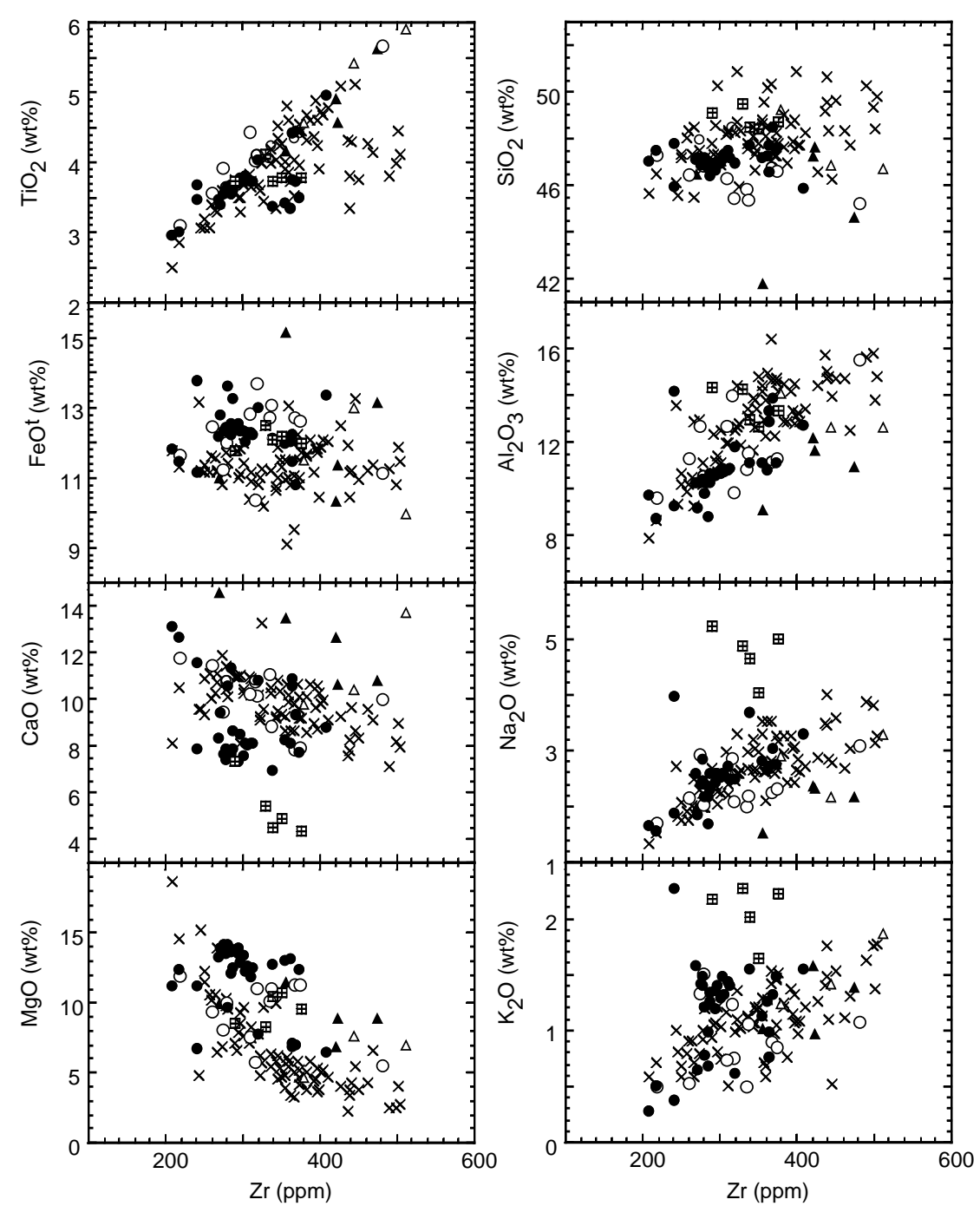

$\boxplus$ Volcaniclastic sediments of subunits 5 and 7 in Hole 956B

- Volcaniclastic sediments in Hole 956B Basalt clasts in Hole 956B

\begin{abstract}
- Volcaniclastic sediments in Hole 954B
$\triangle$ Basalt clasts in Hole 954B

$\times$ Subaerial shield basalts (Heuschkel, 1996)
\end{abstract}

Figure 18. Major element concentration vs. $\mathrm{Zr}$ (ppm) for volcaniclastic sediments and single basalt clasts (Sections 157-954B-35R-1 through 39R-3 and 157-956B-43R-5 through 57R-1). 
Figure 18 (continued). Trace element concentration vs. $\mathrm{Zr}$ (ppm) for volcaniclastic sediments and single basalt clasts (Sections 157-954B-35R-1 through 39R3 and 157-956B-43R-5 through 57R-1).
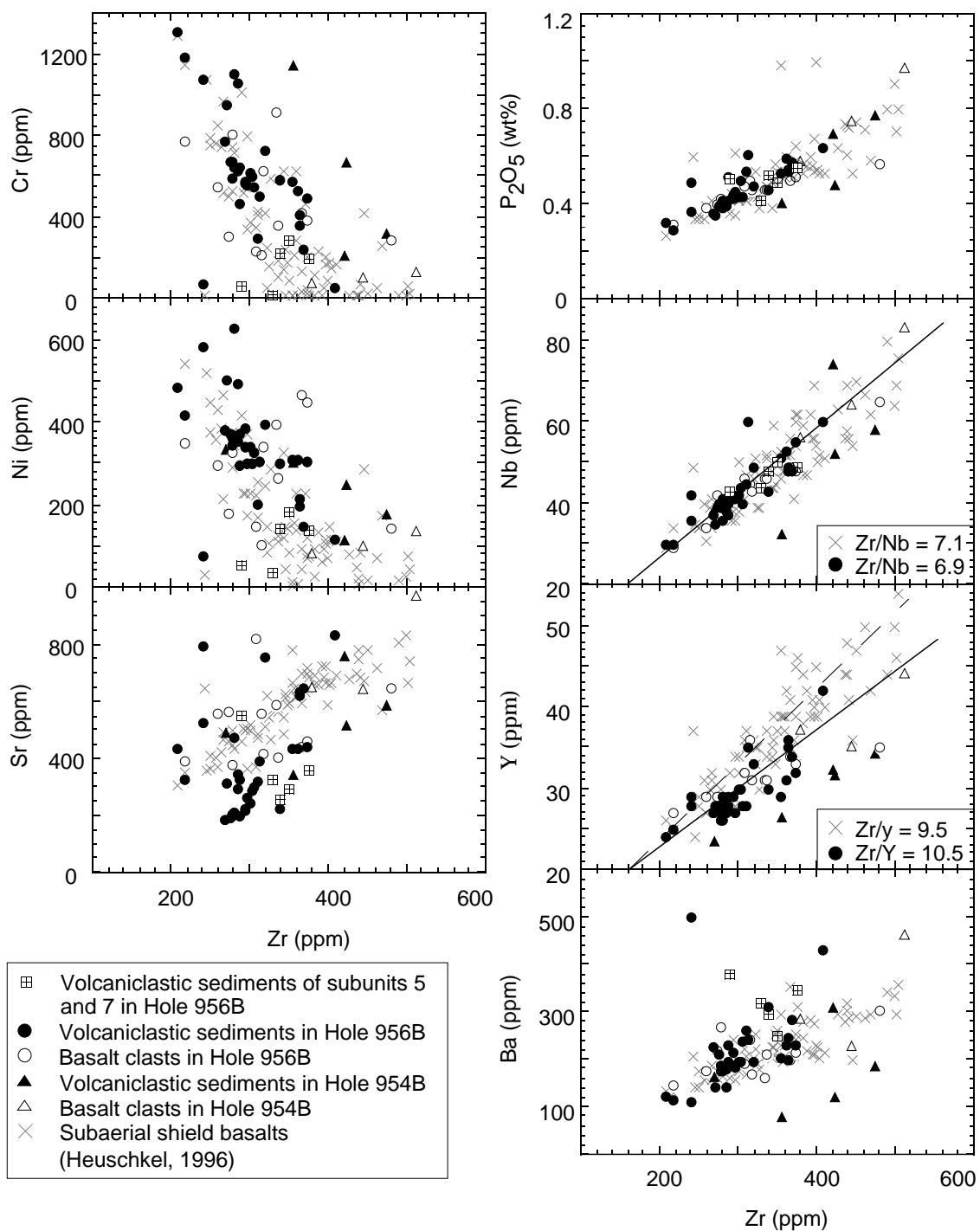
and 7 in Hole 956B

- Volcaniclastic sediments in Hole 956B Basalt clasts in Hole 956B

- Volcaniclastic sediments in Hole 954B Basalt clasts in Hole 954B Subaerial shield basalts (Heuschkel, 1996) $\boxplus \quad$ Volcaniclastic sediments of subunits 5

$r(p p m)$ 
A

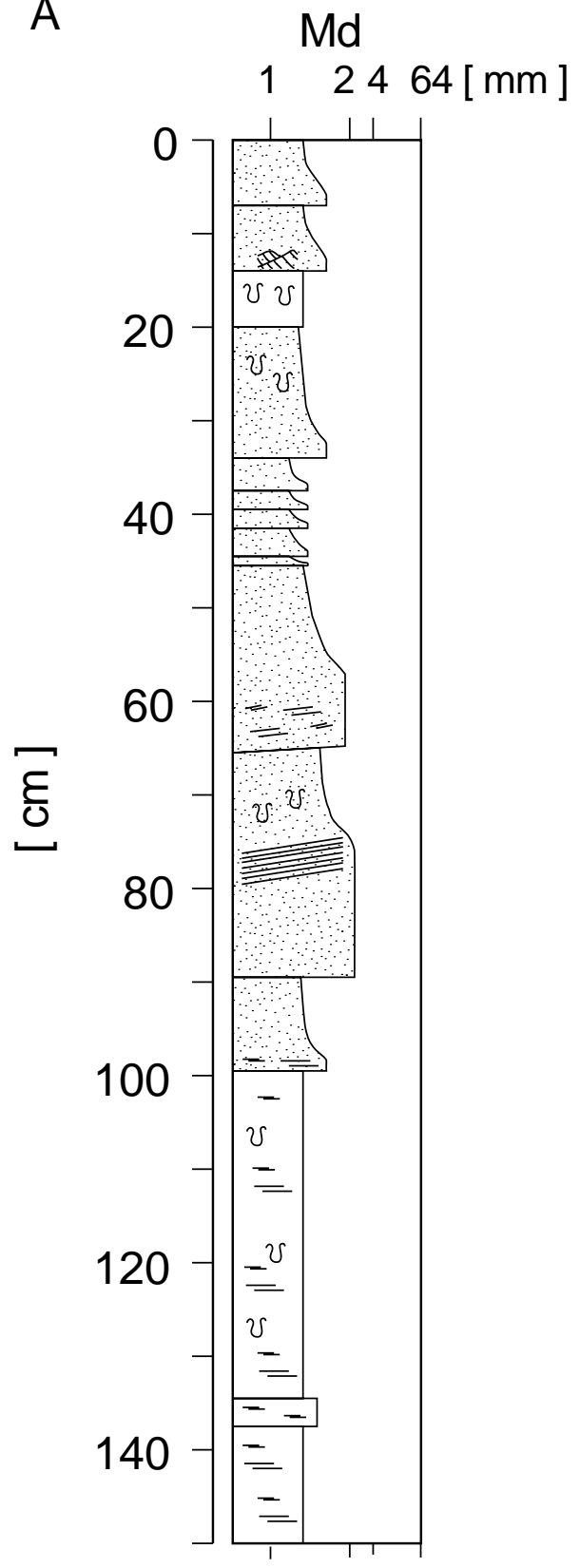

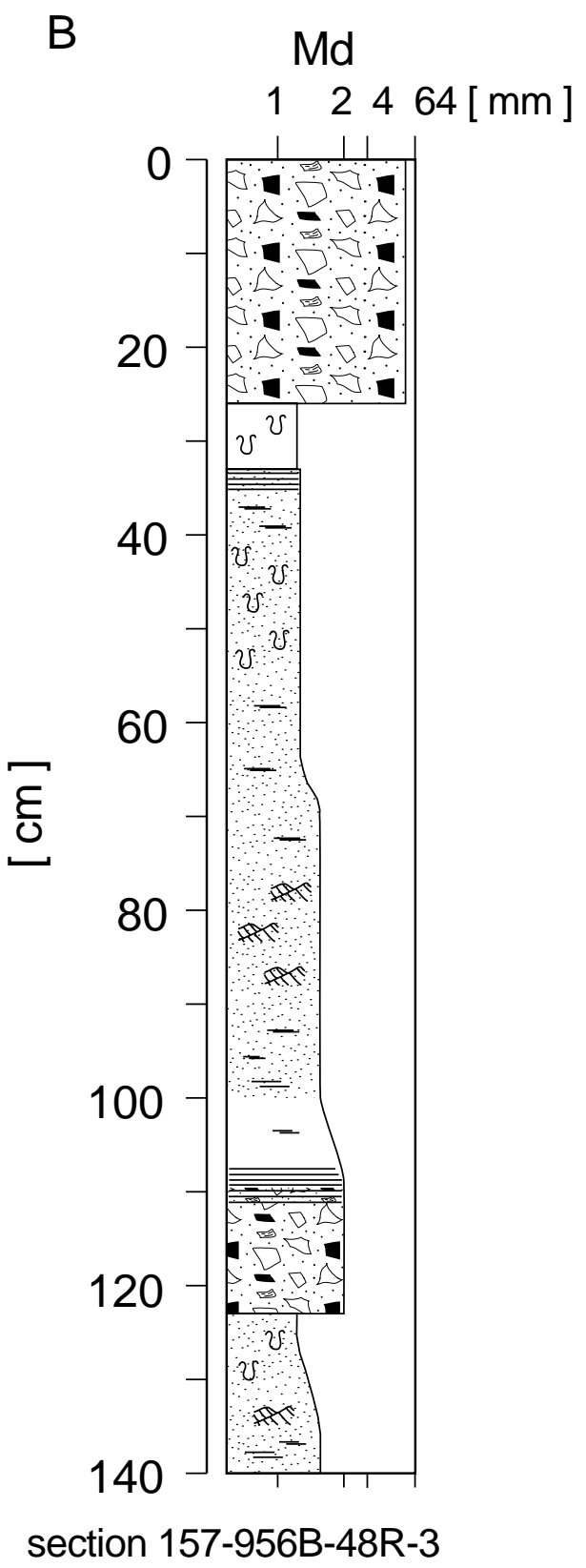

section 157-953C-72R-5

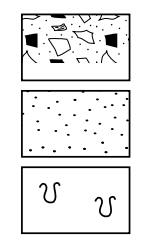

Coarse-grained volcaniclastic sediment

Fine-grained volcaniclastic sediment

Bioturbation

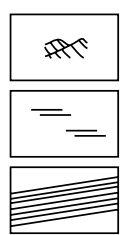

Cross bedding

Diffuse planar stratification

Distinct planar stratification

Figure 19. A. Simplified graphic log of ash turbidites interbedded with nannofossil ooze (Section 157-953C-72R-5). B. Simplified graphic log of graded lithoclasts debris-flow deposits (interval 157-956B-48R-3, $12 \mathrm{~cm}$ ). 

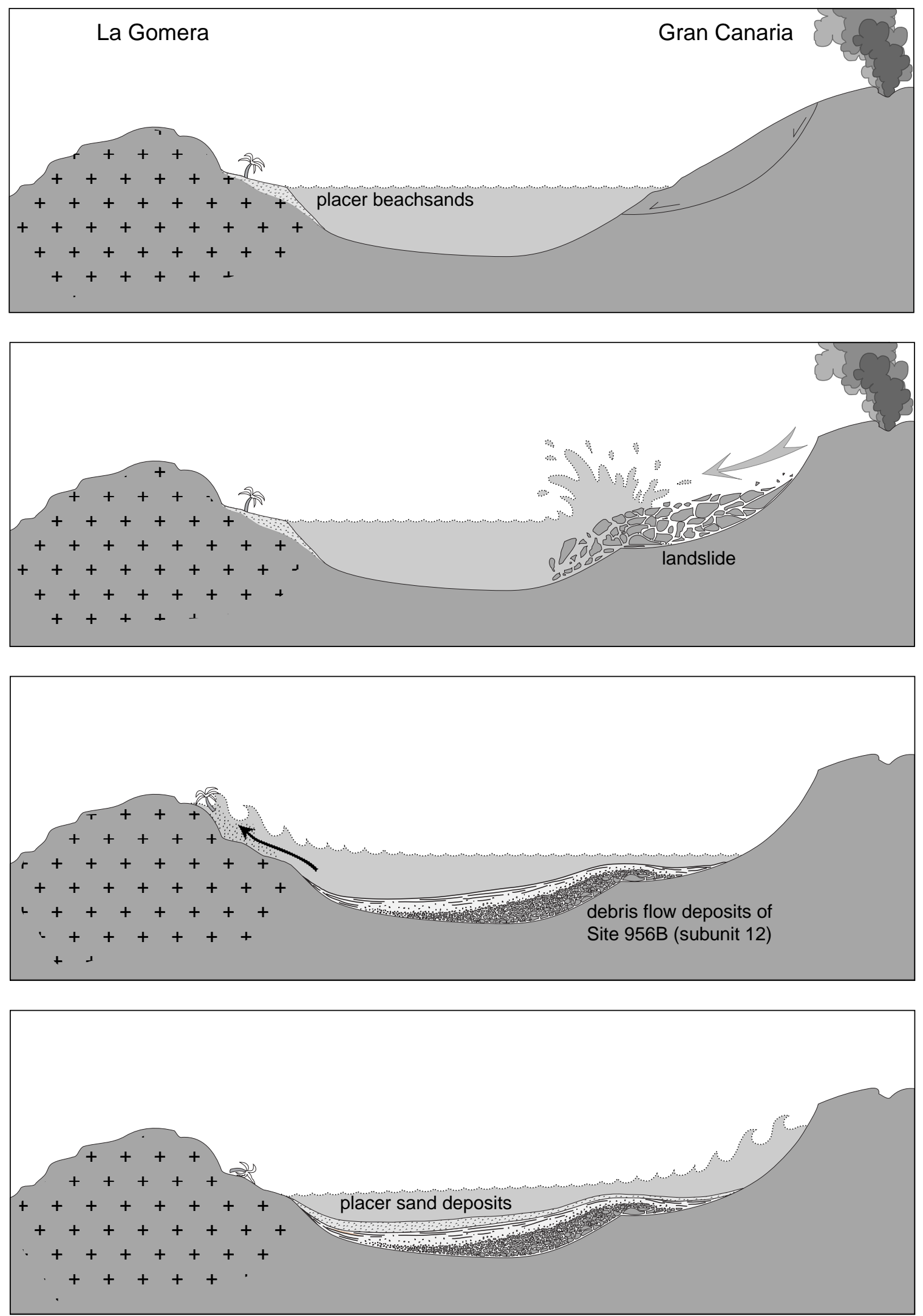

Figure 20. Schematic sketch of the landslide on Gran Canaria, triggering the tsunami deposit of the "placer sandstone" (see text). 
Table 16. Electron microprobe analyses of glass, Hole 956B.

\begin{tabular}{|c|c|c|c|c|c|}
\hline \multirow{2}{*}{$\begin{array}{l}\text { Sample: } \\
\text { Location: }\end{array}$} & \multicolumn{2}{|c|}{ 956B-44R-4, 27-42 cm } & \multirow{2}{*}{$\frac{956 \mathrm{~B}-44 \mathrm{R}-4,110-124 \mathrm{~cm}}{1}$} & \multicolumn{2}{|c|}{$956 \mathrm{~B}-44 \mathrm{R}-5,114-121 \mathrm{~cm}$} \\
\hline & 1 & 2 & & 1 & 2 \\
\hline $\mathrm{SiO}_{2}$ & 50.11 & 48.44 & 49.41 & 50.51 & 50.46 \\
\hline $\mathrm{TiO}_{2}$ & 4.37 & 4.86 & 5.30 & 4.21 & 4.10 \\
\hline $\mathrm{Al}_{2} \mathrm{O}_{3}$ & 12.97 & 12.74 & 12.96 & 13.07 & 13.02 \\
\hline $\mathrm{FeO}$ & 13.37 & 13.66 & 12.09 & 13.11 & 13.11 \\
\hline $\mathrm{MnO}$ & 0.25 & 0.26 & 0.20 & 0.26 & 0.25 \\
\hline $\mathrm{MgO}$ & 4.05 & 4.43 & 4.91 & 4.01 & 3.91 \\
\hline $\mathrm{CaO}$ & 8.20 & 8.84 & 9.17 & 8.09 & 8.05 \\
\hline $\mathrm{Na}_{2} \mathrm{O}$ & 2.97 & 3.01 & 2.87 & 3.14 & 3.07 \\
\hline $\mathrm{K}_{2} \mathrm{O}$ & 1.85 & 1.68 & 1.65 & 1.95 & 1.92 \\
\hline $\mathrm{SO}_{2}$ & 0.02 & 0.02 & 0.03 & 0.00 & 0.03 \\
\hline Total & 98.15 & 97.94 & 98.59 & 98.35 & 97.91 \\
\hline
\end{tabular}

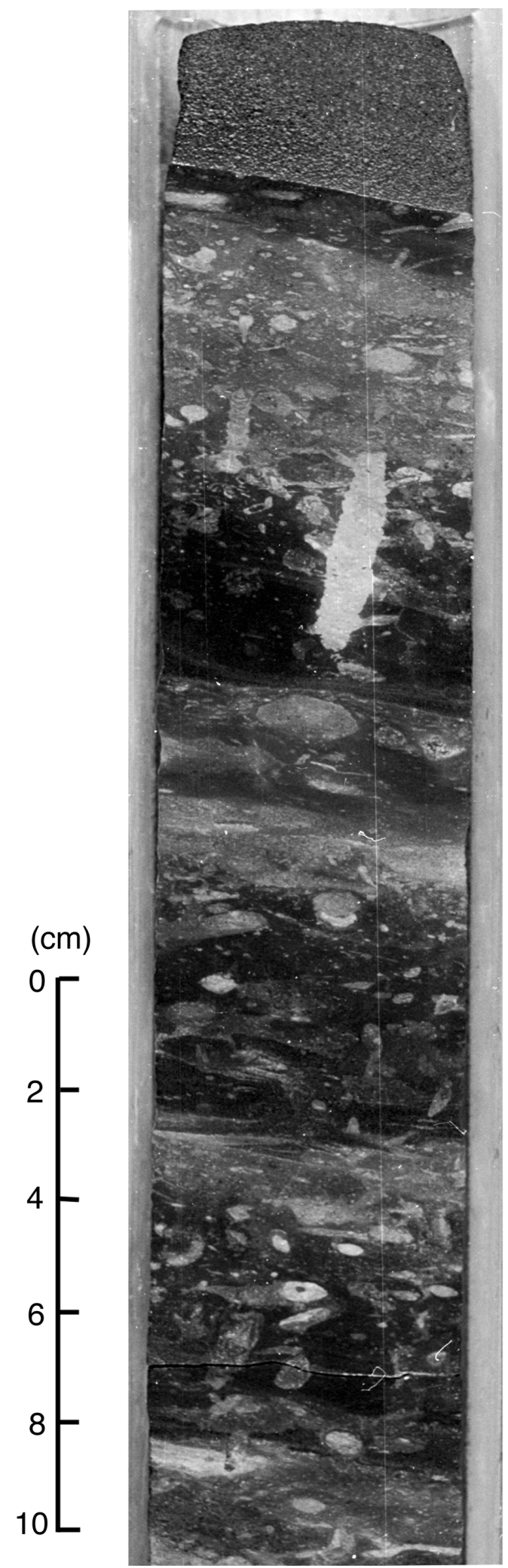

Figure 21. Three basaltic volcaniclastic thin, siltstone turbidites strongly bioturbited near their top overlain by coarser grained tuff (Sample 157-953C73R, 8-37 cm). 


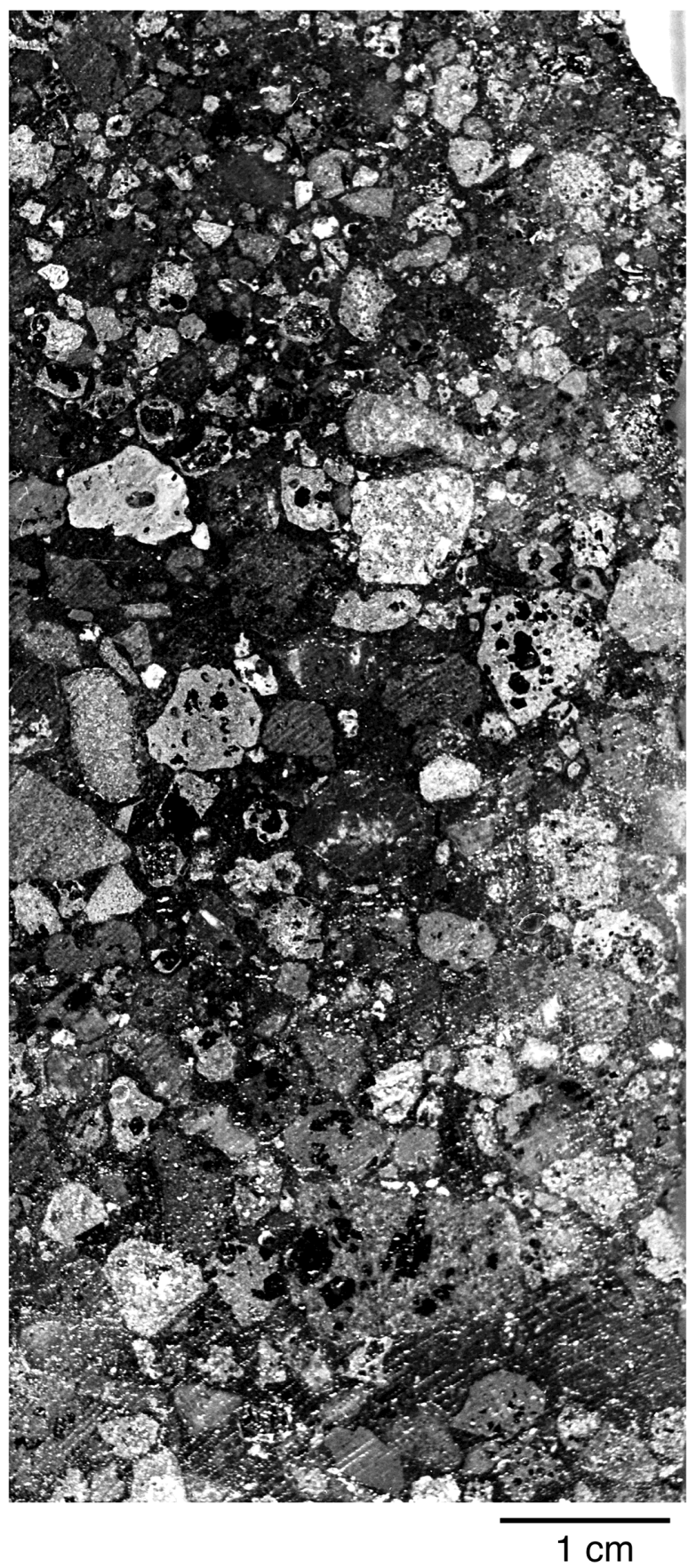

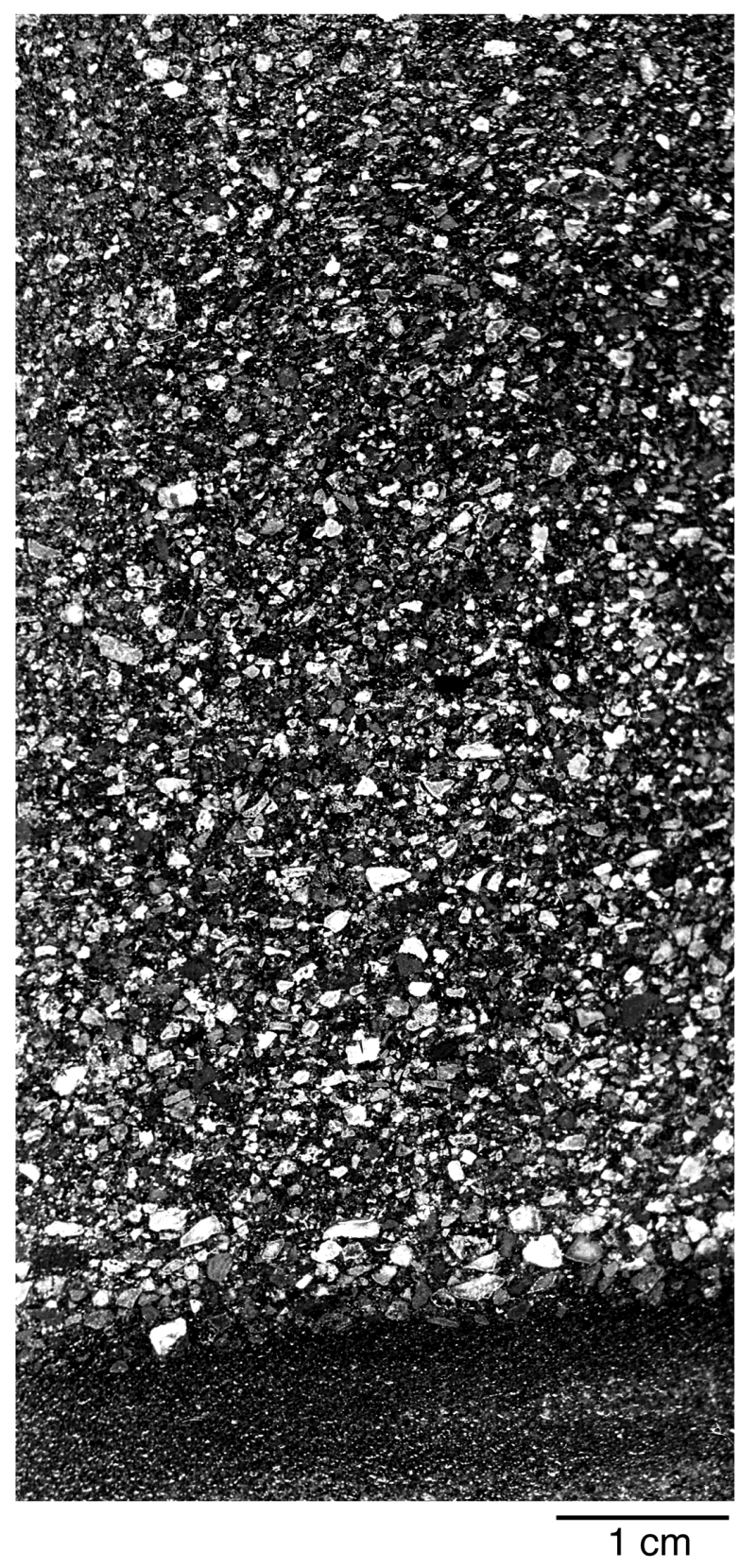

Figure 23. Coarse-grained basaltic tuff, rich in light-colored sideromelane shards, showing sharp basal contact and pronounced grading (Sample 157956B-43R-4, 21-55 cm).

Figure 22. Basaltic volcaniclastic lapillistone in lithologic Unit V showing strong grading and a dark matrix (Sample 157-953C-71R-4, 25-39 cm). 


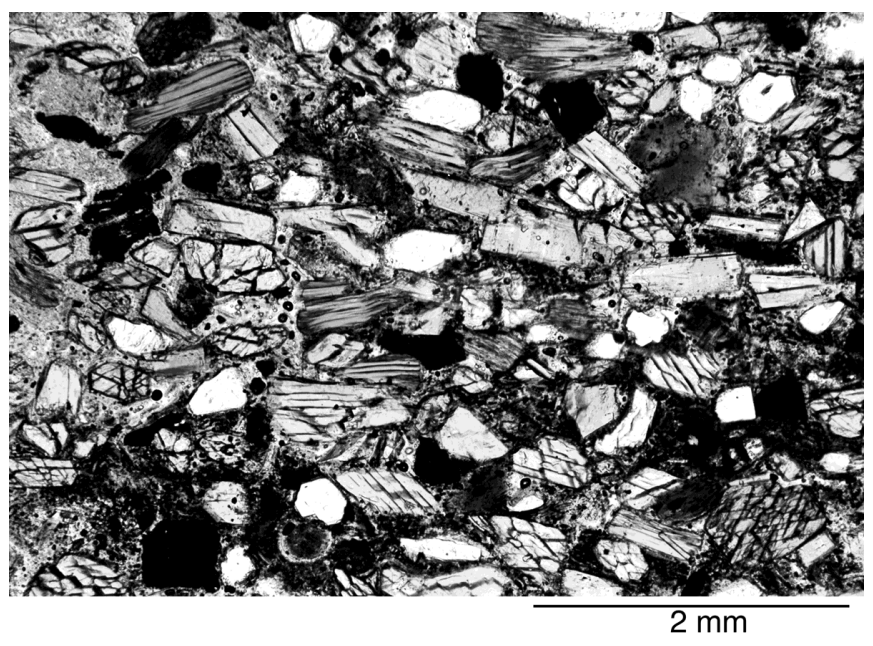

Figure 24. Placer sandstone consisting almost entirely of amphibole, phlogopite, apatite (light-colored crystals), and minor clinopyroxene (Sample 157956B-49R-1, 5-13 cm).

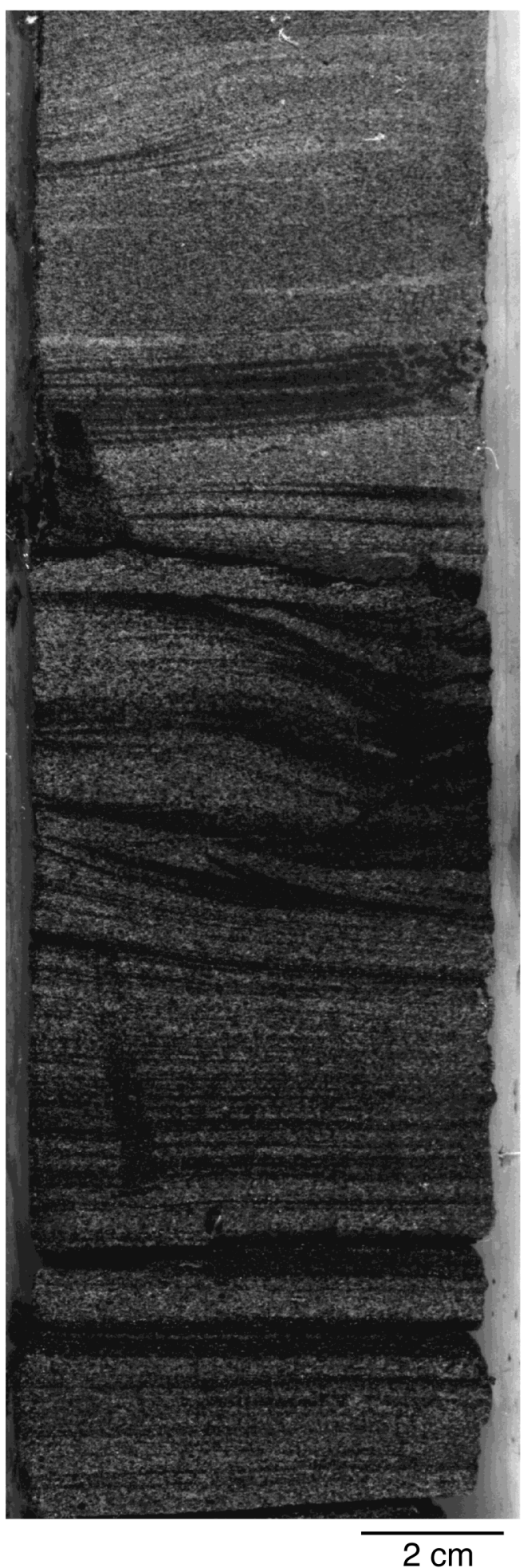

Figure 25. Bedded and cross-bedded sandstone consisting of alternating foraminifer sand (light colored) and placer sands rich in heavy minerals (see Fig. 24; Sample 157-956B-48R-3, 74-93 cm). 


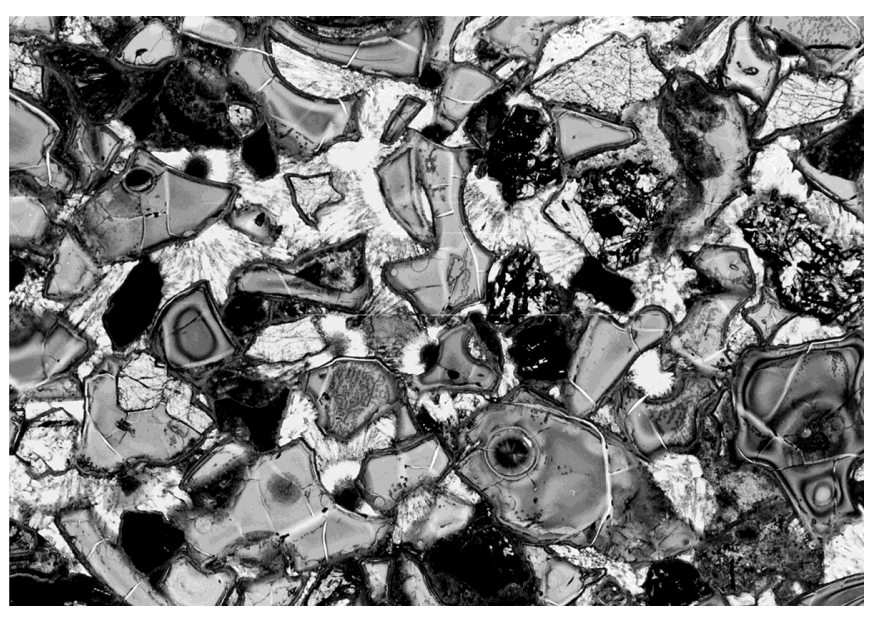

1

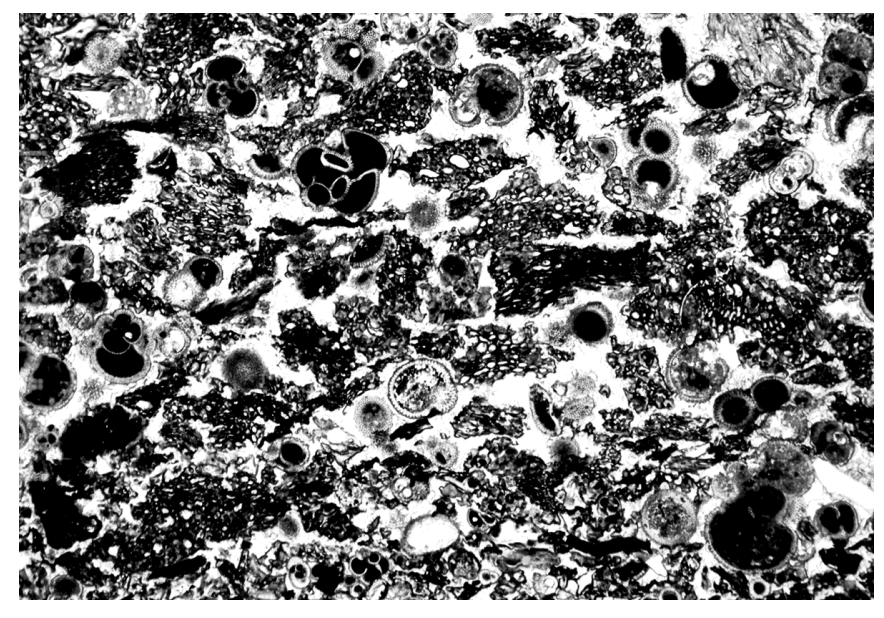

3

$2 \mathrm{~mm}$

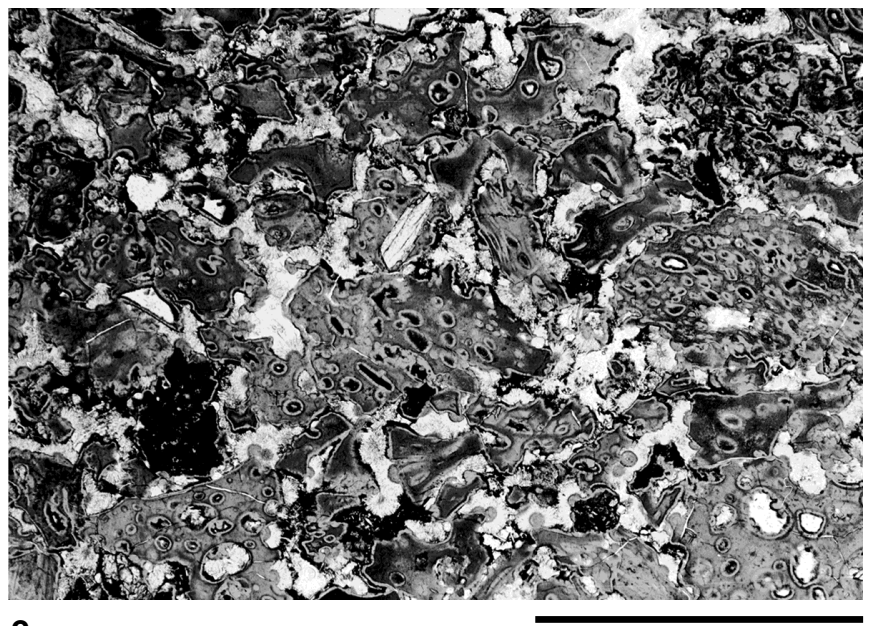

2

$1 \mathrm{~mm}$

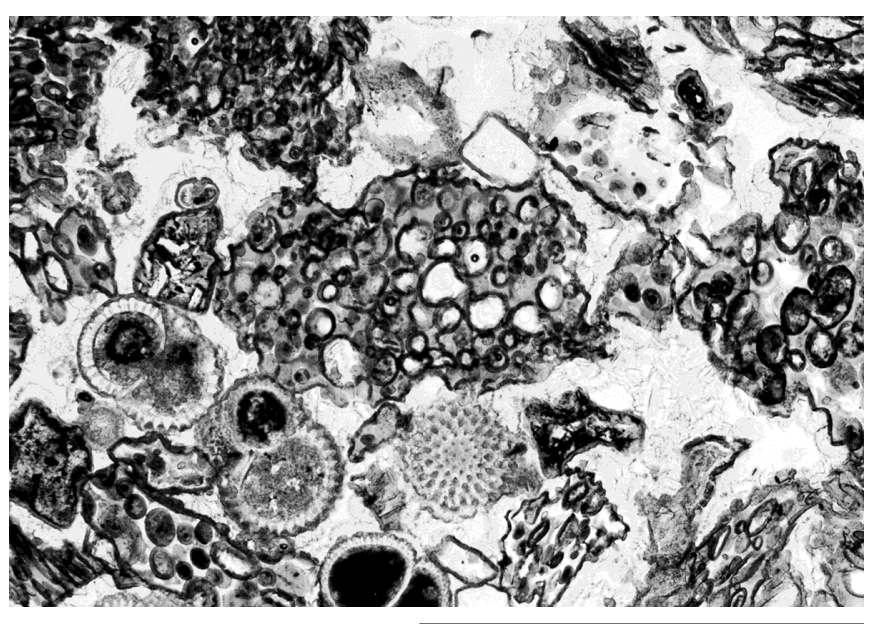
4

$1 \mathrm{~mm}$

Plate 1. 1. Blocky altered sideromelane shards with marginal traces of a few large vesicles. A few dark tachylites and crystalline basalt clasts are interspersed with tachylite. Clinopyroxene phenocrysts in some sideromelane shards. The pore space is filled with phillipsite (Sample 157-953C-103R-2, 42-52 cm). 2. Moderately vesicular shards with smaller vesicles in the upper part of lithologic Unit VII (Sample 157-953C-83R-6, 107-120 cm). 3. Very strongly vesicular pumiceous altered shards interspersed with planktonic foraminifers. Pore space filled by phillipsite (Sample 157-956B-43R-5, 14-21 cm). 4. Highly vesicular shards and filled planktonic foraminifers set in a phillisite cement (Sample 157-956B-43R-5, 14-21 cm). 


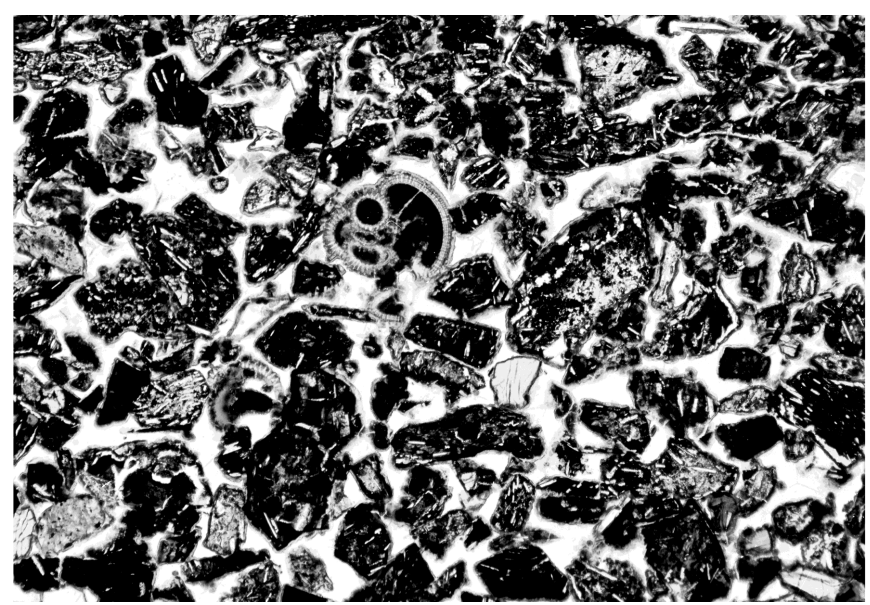

1

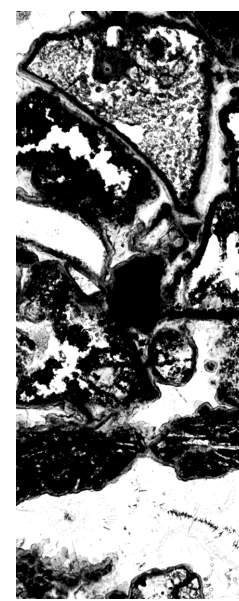

3

$1 \mathrm{~mm}$
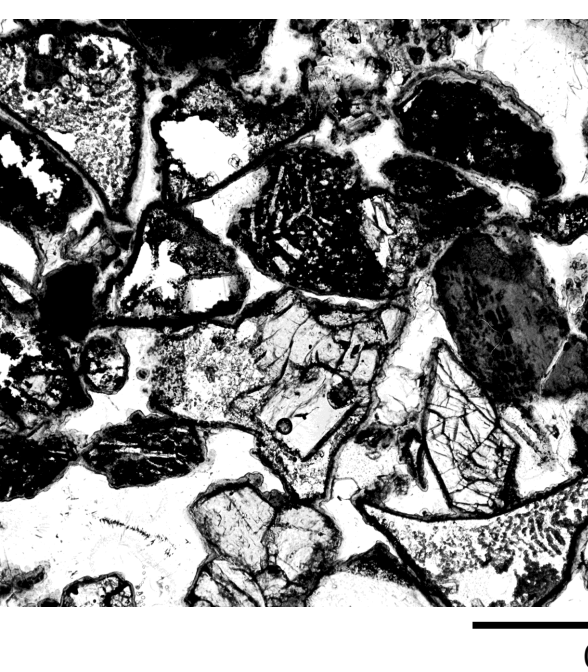
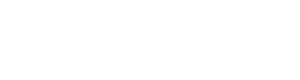

Plate 2. 1. Basaltic lithic sandstone composed almost entirely of tachylite clasts with plagioclase microlites and a few filled planktonic foraminifers. Pore space filled by zeolites (Sample 157-956B-43R-8, 108-114 cm). 2. Mixed lithic basaltic sandstone composed of angular tachylite, proxene, and plagioclase crystals, crystalline basalt clasts, and minor altered sideromelane clasts set in a matrix of clay and zeolites (Sample 157-956B-49R-3, 54-63 cm). 3. Basaltic volcaniclastic sandstone composed of completely altered angular shards, some with clinopyroxene phenocrysts, tachylite, and crystalline basalts, the pore space being filled by phillipsite (Sample 157-956B-45R-3, 86-90 cm). 4. Matrix-supported (brown clay) basaltic lithic sandstone composed of angular tachylite clasts, crystalline basalt fragments, completely altered light-colored sideromelane shards, and large clinopyroxene crystals (Sample 157-956B-51R-3, 97-123 cm). 


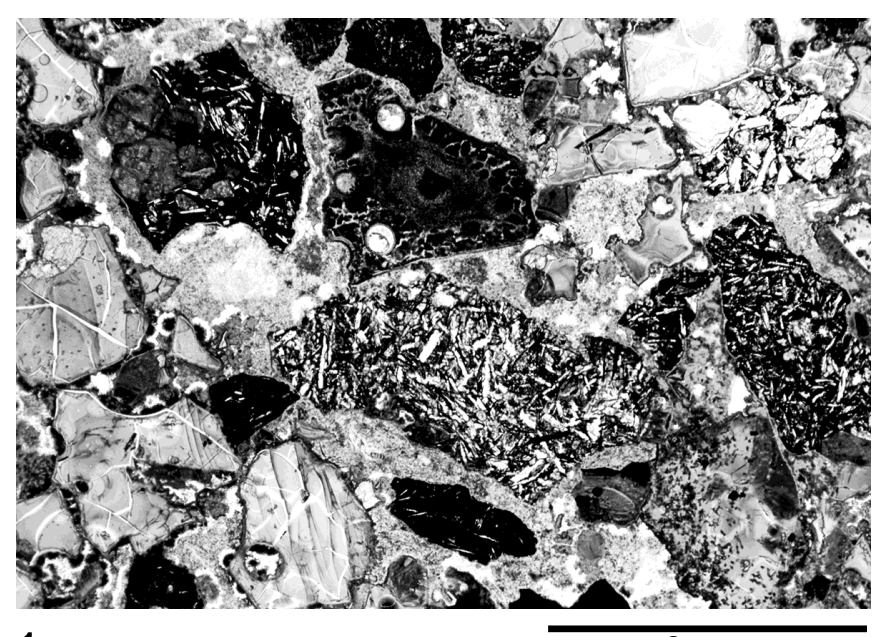

1

$2 \mathrm{~mm}$
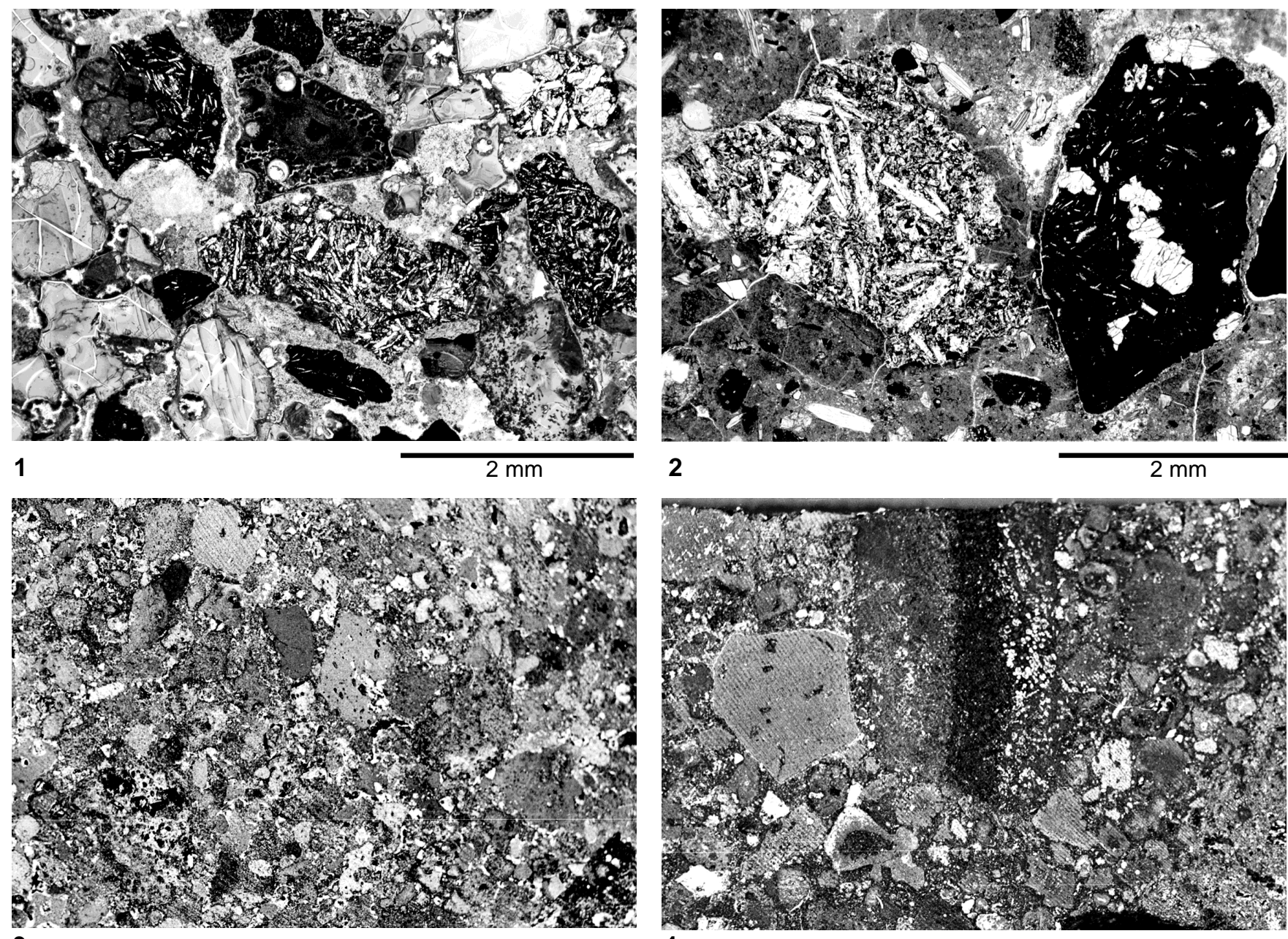

3

$1 \mathrm{~cm}$

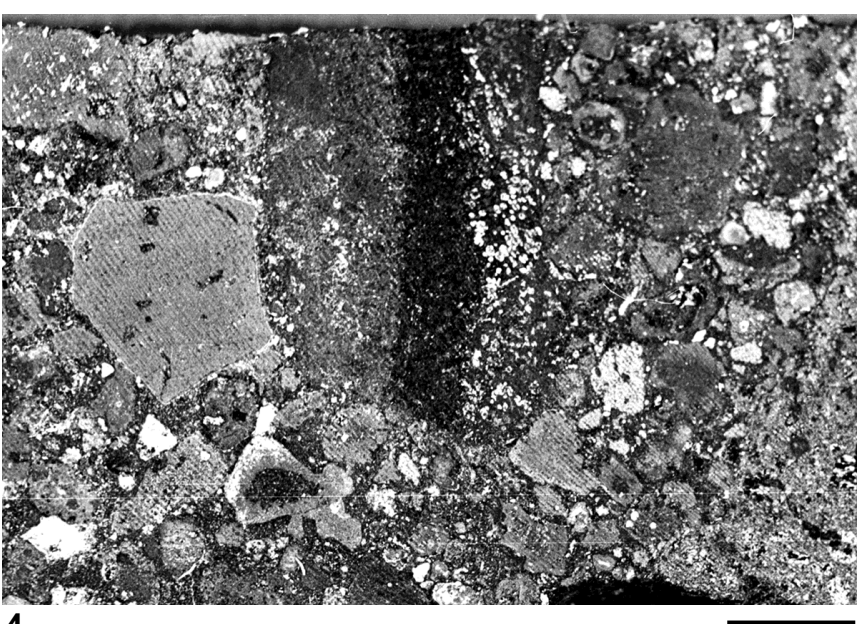

$1 \mathrm{~cm}$

Plate 3. 1. Volcaniclastic sandstone composed of blocky sideromelane shards showing traces of large diameter vesicles, angular, partly vesicular tachylite and three fragments of crystalline basalt ranging from fine- to coarse-grained (Sample 157-953C-98R-1, 0-12 cm). 2. Basaltic lithic sandstone showing one large tachylite and one crystalline basalt fragment and small crystals of xenocrystic amphibole and phlogopite between the two large clasts. Dark matrix is dominantly brown clay. 3. Basaltic lithic lapillistone, clast-supported (Sample 157-956B-46R-4, 12-24 cm). 4. Basaltic breccia and lapillistone showing one large fragment of basalt and smaller marginal fragments of pillow with slightly vesicular altered rim. Note angularity of clasts (Sample 159-956B-48R-2, 26-43 cm). 


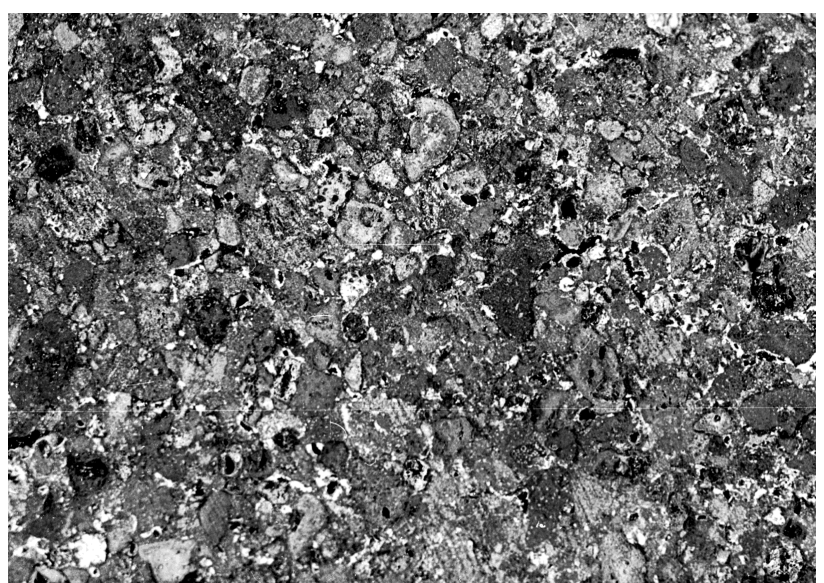

$1 \mathrm{~cm}$
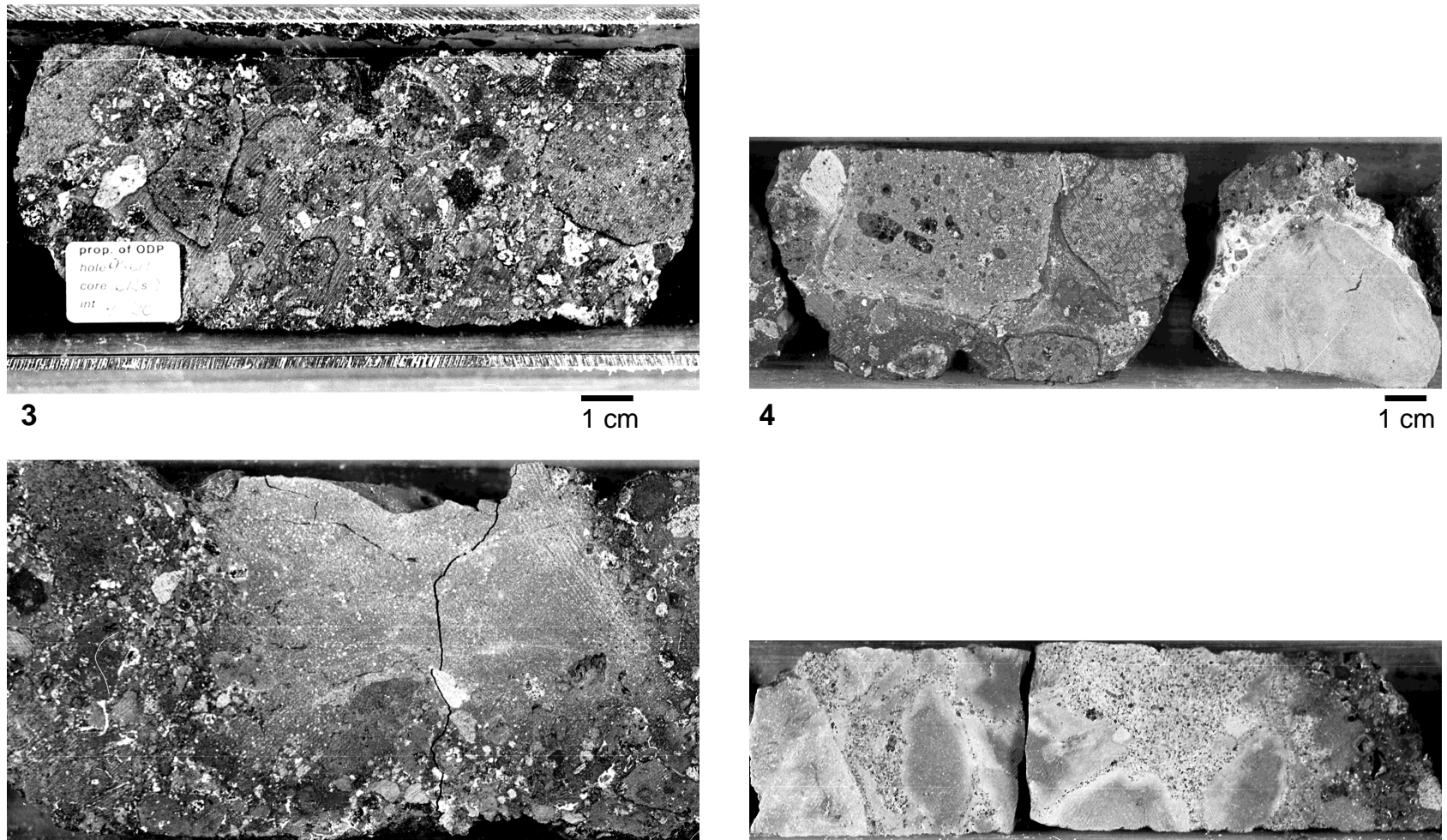

5

$1 \mathrm{~cm}$

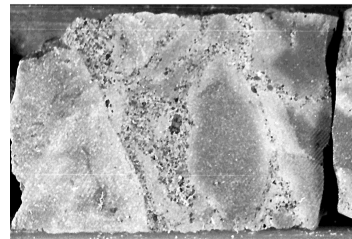

6

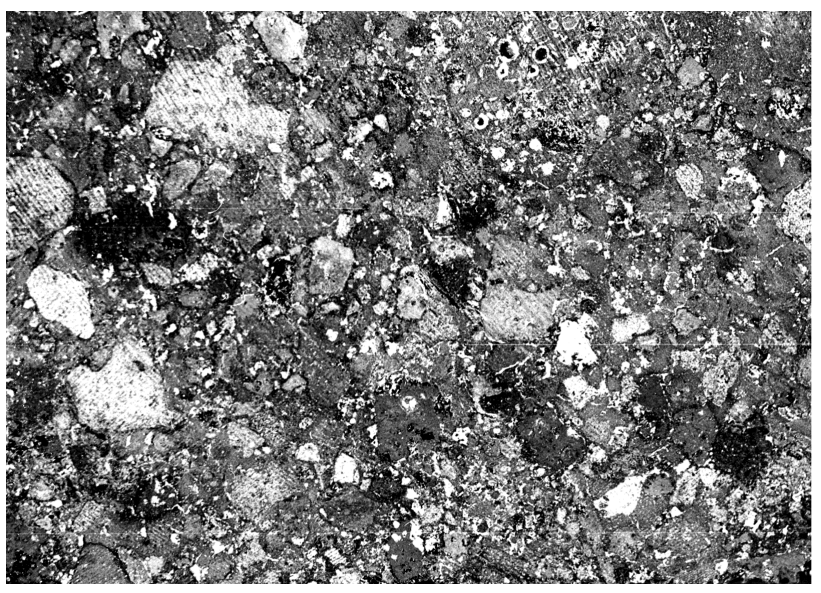

2

$1 \mathrm{~cm}$

Plate 4. 1. Relatively well-sorted basaltic lithic lapillistone with pores being partly filled by white zeolites. Upper part of very thick basal debris flow (Sample 157-956B-51R-5, 0-2 cm). 2. Increasing grain size downward in basal debris flow at Hole 956B. Note 2-cm-large very vesicular oxidized basalt fragment with partially filled vesicles (Sample 157-956B-53R-2, 77-79 cm). 3. Poorly lithic breccia in basal debris flow from Hole 956B (Sample 157-956B-56R-3, 8-20 cm). 4. Lower part of basal debris flow from Hole 956B showing angular to well-rounded fragments of basalt set in a finer grained matrix (Sample 956B-956B-57R-1, 12-21 cm). 5. Deformed pelagic sediment pick-up clasts in the interior of basal debris flow from Hole 956B (Sample 157-956B-53R-2, 55-65 cm). 6. Highly deformed and brecciated large pelagic sediment pick-up clasts in the interior of basal debris flow from Hole 956B (Sample 157-956B-35R-3, 37-63 cm). 